\title{
Determination of the $J$-groups of Complex Projective and Lens Spaces
}

\section{DIBAG}

Department of Mathematics, Bilkent University, 06800 Ankara, Turkey. e-mail:dibag@fen.bilkent.edu.tr

(Received: April 2002)

Abstract. We determine completely the $J$-groups of complex projective and lens spaces by means of a set of generators and a complete set of relations.

Mathematics Subject Classifications (2000): Primary 55R25; secondary 55Q50, 55S25.

Key words: sphere bundles, vector bundles, $J$-morphism, $K$-Theory operations.

\section{Introduction}

For a finite-dimensional CW-complex $X$, let $J(X)$ denote the finite Abelian group of stable fibre homotopy classes of vector bundles over $X$ and for a prime $p, J_{p}(X)$, the $p$-summand of $J(X)$. For $n, k \in \mathbb{Z}^{+}$, let $P_{n}(\mathbb{C})=S^{2 n+1} / U(1)$ and $L^{n}\left(p^{k}\right)=$ $S^{2 n+1} / \mathbb{Z}_{p^{k}}$ denote the complex projective space of (complex) dimension $n$ and the associated lens space, respectively. The aim of this paper is to determine $J_{p}\left(P_{n}(\mathbb{C})\right)$ and $J\left(L^{n}\left(p^{k}\right)\right)$ by means of a set of generators and a complete set of relations. Let $r_{n}$ be the greatest integer such that $p^{r_{n}} \leqslant n /(p-1)$. Then for $0 \leqslant s \leqslant r_{n}$ and $0 \leqslant j \leqslant r_{n}-s$, we define the integer

$$
t_{j}^{s}=\left[\frac{n-p^{s}\left(p^{j}-1\right)}{p^{s+j}(p-1)}\right] \text {. }
$$

Let $\eta$ denote the complex Hopf bundle over $P_{n}(\mathbb{C})$ and $\omega=r(\eta)-2 \in \tilde{K}_{\mathbb{R}}\left(P_{n}(\mathbb{C})\right)$, where $r$ denotes realification and also denote by $\omega$ the pull-back of $\omega$ to $\tilde{K}_{\mathbb{R}}\left(L^{n}\right.$ $\left.\left(p^{k}\right)\right)$. It is proved in [10] that the Adams operation $\psi_{\mathbb{R}}^{k}$ passes to the quotient and acts on $J(X)$ and that $\psi_{\mathbb{R}}^{k}=1$ on $J_{p}(X)$ for $(k, p)=1$. It follows from this and the results of [4] on $J\left(P_{n}(\mathbb{C})\right)$ that $J_{p}\left(P_{n}(\mathbb{C})\right)$ is generated by $\left\{\omega, \psi_{\mathbb{R}}^{p}(\omega), \ldots, \psi_{\mathbb{R}}^{p^{r_{n}}}\right.$ $(\omega)\}$. We seek $\left(r_{n}+1\right)$-relations between these generators of the form:

$$
\sum_{j=0}^{r_{n}-s} \alpha_{j}^{s} p^{t_{j}^{s}} \psi_{\mathbb{R}}^{p^{s+j}}(\omega)=0 \quad\left(0 \leqslant s \leqslant r_{n}\right),
$$

where the integers $\alpha_{j}^{s}$ are to be determined. Let $\rho_{\mathbb{R}}^{k}$ denote the $K$-theory characteristic classes associated to the Adams operation $\psi_{\mathbb{R}}^{k}$ introduced in [1, Section 5] 
(also denoted by $\Theta_{k}$ in [7]). We prove a key result (e.g. Proposition 2.2.1) to the effect that if $\tilde{K}_{\mathbb{R}}(X)$ is $p$-torsion free then $J_{p}(x)=0$ iff $\rho_{\mathbb{R}}^{p}(x)$ is integral. Thus, for $p$ odd or $p=2$ and $n \not \equiv 1(\bmod 4)$,

$$
\sum_{j=0}^{r_{n}-s} \alpha_{j}^{s} p^{t_{j}^{s}} \psi_{\mathbb{R}}^{p^{s+j}}(\omega)=0 \quad \text { in } J_{p}\left(P_{n}(\mathbb{C})\right)
$$

if and only if

$$
\rho_{\mathbb{R}}^{p}\left(\sum_{j=0}^{r_{n}-s} \alpha_{j}^{s} p^{t_{j}^{s}} \psi_{\mathbb{R}}^{p^{s+j}}(\omega)\right)=\prod_{j=0}^{r_{n}-s} \rho_{\mathbb{R}}^{p}\left(\psi_{\mathbb{R}}^{p^{s+j}}(\omega)\right)^{\alpha_{j}^{s} p^{t_{j}^{s}}} \text { is integral. }
$$

We let

$$
\rho_{\mathbb{R}}^{p}\left(\psi_{\mathbb{R}}^{p^{s+j}}(\omega)\right)^{p^{t_{j}^{s}}}=1+\sum_{m=1}^{[n / 2]} b_{m}^{s, j} \omega^{m}
$$

$m$ is called a singular $s$-exponent if and only if $b_{m}^{s, j} \notin \mathbb{Z}$ for some $j$. We denote the set of singular $s$-exponents by $\mathcal{M}_{n, s}$. We define the $(s, j)$-index of $m(m \geqslant 1)$ which is an integer denoted by $\varepsilon_{m}^{s, j}$ and prove that $m \in \mathcal{M}_{n, s}$ if and only if $\varepsilon_{m}^{s, j}>0$ for some $j \in \mathbb{Z}^{+}$. If $m \in \mathcal{M}_{n, s}$, then $m$ can be written in the form, $m=(1 / 2)$ $(p-1) p^{s+i} \Delta(i \geqslant 1,(\triangle, p)=1)$. We define

$$
\Phi_{n, s}=\left\{t_{j}^{s}: 0 \leqslant j \leqslant r_{n}-s\right\}, \quad \Phi_{n, s}^{0}=\left\{t_{j}^{s} \in \Phi_{n, s}: t_{j}^{s} \equiv 0 \quad(\bmod p) .\right.
$$

If $t_{k}^{s}=p^{\nu} \Delta \in \Phi_{n, s}^{0}(v \geqslant 1,(\Delta, p)=1)$ then $\left(t_{j}^{s}\right)_{k+1 \leqslant j \leqslant k+\nu}=\left(p^{\nu-1} \Delta-1\right.$, $\left.p^{\nu-2} \triangle-1, \ldots, \Delta-1\right)$. Thus, if we denote $T_{k}^{s}=\left(t_{k}^{s}, t_{k+1}^{s}, \ldots, t_{k+\nu}^{s}\right)$ then for consecutive elements $t_{k}^{s}, t_{l}^{s} \in \Phi_{n, s}^{0}(k<l), T_{k}^{s} \bigcap T_{l}^{s}$ is either empty or equal to $\left\{t_{l}^{s}\right\}$ and the latter is always the case if $p=2$. A key result is Proposition 4.4.4 which states that there exists a bijection, $\sigma: \Phi_{n, s}^{0} \rightarrow \mathcal{M}_{n, s}$ given by

$$
\sigma\left(t_{k}^{s}\right)=(1 / 2)(p-1) p^{s+k} t_{k}^{s}
$$

If

$$
t_{k}^{s}=p^{v} \Delta(v \geqslant 1,(\Delta, v)=1) \quad \text { and } \quad m=\sigma\left(t_{k}^{s}\right)=(1 / 2)(p-1) p^{s+k+v} \Delta
$$

then

(1) $\left(\varepsilon_{m}^{s, j}\right)_{k \leqslant j \leqslant k+v}=(v v v-1 v-2 \cdots 1)$ (e.g. Lemma 4.4.3).

(2) $\left(\varepsilon_{m}^{s, j}\right)_{j \leqslant k-1}$ is a strictly increasing sequence bounded above by $v$ (e.g. Lemma 4.4.7).

and as a consequence of (1) and (2) we have the

OBSERVATION 1.1.1. Given $j \leqslant k$ such that $\varepsilon_{m}^{s, j}>0$, then there exists a unique $k+1 \leqslant j^{\prime} \leqslant k+v$ such that $\varepsilon_{m}^{s, j}=\varepsilon_{m}^{s, j^{\prime}}$. 
We prove in Section 4.3 a number of integrality theorems as a result of which it follows that if $\hat{Q}_{p}$ is the sub-ring of rationals which are $p$-integral then

(1) $b_{m}^{s, j} \equiv \mp(1 / \Delta)\left(1 / p^{\varepsilon_{m}^{s, j}}\right)\left(\bmod \hat{Q}_{p}\right)($ e.g. Corollary 4.5.2).

(2) If $\prod_{j=0}^{r_{n}-s} \rho_{\mathbb{R}}^{p}\left(\psi_{\mathbb{R}}^{p^{s+j}}(\omega)\right)^{\alpha_{j}^{s} p^{t_{j}^{s}}}=1+\sum_{m \geqslant 1} c_{m} \omega^{m}$ then $c_{m} \in \mathbb{Z}$ if $m \notin \mathcal{M}_{n, s}$ and if $m \in \mathcal{M}_{n, s}$, then $c_{m} \equiv \mp(1 / \triangle) \sum_{j}\left(\alpha_{j}^{s} / p^{\varepsilon_{m}^{s, j}}\right)\left(\bmod \hat{Q}_{p}\right)$ if either $p \neq 2$ or $m$ is not the least element of $\mathcal{M}_{n, s}$ and

$$
c_{m} \equiv-\frac{1}{\triangle} \sum_{\substack{0 \leqslant j \leqslant i \\ j \neq i-1}} \frac{\alpha_{j}^{s}}{2^{s, j}}+\frac{1}{\triangle} \frac{\alpha_{i-1}^{s}}{2^{\varepsilon_{m}^{s, i-1}}} \quad\left(\bmod \hat{Q}_{p}\right)
$$

if $p=2$ and $m$ is the least element of $\mathcal{M}_{n, s}$ (i.e. Corollary 4.5.3). For $0 \leqslant s \leqslant$ $r_{n}$, we define $\alpha^{s}=\left(\alpha_{j}^{s}\right)_{0 \leqslant j \leqslant r_{n}-s}$ to be an $s$-admissible sequence if and only if $\sum_{j} \alpha_{j}^{s} / p^{\varepsilon_{m}^{s, j}} \in \mathbb{Z} \forall m \in \mathcal{M}_{n, s}$. Except for $p=2$ and $m$ is the least element of $\mathcal{M}_{n, s}$ in which case it is required that

$$
\sum_{\substack{0 \leq j \leq i \\ j \neq i-1}} \frac{\alpha_{j}^{s}}{2^{\varepsilon_{m}^{s, j}}}-\sum \frac{\alpha_{i-1}^{s}}{2^{\varepsilon_{m}^{s i-1}}} \in \mathbb{Z} .
$$

Then it follows from Proposition 2.2.1 and the above that $\sum_{j=0}^{r_{n}-s} \alpha_{j}^{s} p^{t_{j}^{s}}\left(\psi_{\mathbb{R}}^{p^{s+j}}\right.$ $(\omega))=0$ in $J_{p}\left(P_{n}(\mathbb{C})\right)$ if and only if $\alpha^{s}=\left(\alpha_{j}^{s}\right)$ is an $s$-admissible sequence. We extend this result to the case $p=2$ and $n \equiv 1(\bmod 4)$ where 2 -torsion exists in $\tilde{K}_{\mathbb{R}}\left(P_{n}(\mathbb{C})\right)$. Using the Observation stated above and by ordering the singular exponents and using induction, two $s$-admissible sequences, called $\alpha$ - and $\beta$ sequences are constructed and we obtain the corresponding $\left(r_{n}+1\right)$-relations in $J_{p}\left(P_{n}(\mathbb{C})\right)$. Of these, the $\alpha$-relation has the advantage that the sequence consists only of 0 and \pm 1 . Both of these relations are complete except for $p=2$ and $n \equiv 3(\bmod 4)$ in which case the complete set of relations is given by the corresponding $\alpha$ - and $\beta$-relations for $(n-1)$ via the isomorphism, $J_{2}\left(P_{n}(\mathbb{C})\right)=J_{2}\left(P_{n-1}(\mathbb{C})\right)$.

The subgroup $G(p, n, k)$ of the $J$-group of the lens space $L^{n}\left(p^{k}\right)$ generated by powers of $\omega$, or, equivalently, by the images of the Adams operations acting on $\omega$, is a quotient of $J_{p}\left(P_{n}(\mathbb{C})\right)$ with the relation, $\psi_{\mathbb{R}}^{p^{k}}(\omega)=0$. Hence, a complete set of relations on $G(p, n, k)$, called the fundamental relations, is given by either the $\alpha$ or $\beta$-relations with $\psi_{\mathbb{R}}^{p^{k}}(\omega), \psi_{\mathbb{R}}^{p^{k+1}}(\omega), \ldots$ all omitted.

(1) If $p$ is odd,

$$
J\left(L^{n}\left(p^{k}\right)\right)= \begin{cases}G(p, n, k) & \text { if } n \neq 0(\bmod 4), \\ G(p, n, k) \oplus \mathbb{Z}_{2} & \text { if } n \equiv 0(\bmod 4) .\end{cases}
$$

(2) If $p=2$, let $\xi$ be the non-trivial real line bundle over $L^{n}\left(2^{k}\right)$ such that

$$
c(\xi)=\psi_{\mathbb{C}}^{2^{k-1}}(\eta) \quad \text { and } \quad \lambda=\xi-1 \in \tilde{K}_{\mathbb{R}}\left(L^{n}\left(2^{k}\right)\right) .
$$


We have

$$
\begin{aligned}
2 \lambda & =r c(\lambda)=r c(\xi)-2=r\left(\psi_{\mathbb{C}}^{2^{k-1}}(\eta)\right)-2=\psi_{\mathbb{R}}^{2^{k-1}}(r(\eta))-2 \\
& =\psi_{\mathbb{R}}^{2^{k-1}}(r(\eta)-2)=\psi_{\mathbb{R}}^{2^{k-1}}(\omega) .
\end{aligned}
$$

Then $J\left(L^{n}\left(2^{k}\right)\right)$ is generated additively by $\lambda$ and $G(p, n, k)$ with the relation $2 \lambda=\psi_{\mathbb{R}}^{2^{k-1}}(\omega)$. Hence, the fundamental relations together with this relation yields a complete set of relations on $J\left(L^{n}\left(2^{k}\right)\right)$.

As a consequence of the $\alpha$-relations for the complex projective space, we prove (e.g. Proposition 6.2.10) that $\mathcal{J}(p, n, k)=\psi_{\mathbb{R}}^{p^{k}}\left(J_{p}\left(P_{n}(\mathbb{C})\right)\right)$ is isomorphic to $J_{p}\left(P_{\left[n / p^{k}\right]}(\mathbb{C})\right)$. This furnishes an alternative proof to Proposition 3.3.1 that the $J_{p}$-order of $\psi_{\mathbb{R}}^{p^{k}}(\omega)$ in $J_{p}\left(P_{n}(\mathbb{C})\right)$ is the $p$-component, $M_{\left[n / p^{k}\right]+1}, p$ of the Atiyah-Todd number $M_{\left[n / p^{k}\right]+1}$. As a corollary of Proposition 3.3.1, follows a very simple proof of Lam's result which is stated in [20] without proof. For $n=(p-1) p^{k}+r(0 \leqslant r \leqslant p-2)$, the $\alpha$-relation takes an overwhelmingly simple form (e.g. Proposition 6.2.12) from which we immediately deduce the primary decomposition of $J_{p}\left(P_{n}(\mathbb{C})\right)$ as a direct-sum of cyclic groups (e.g. Proposition 6.2.13). As a demonstration of how simply the relations can be computed, the case of $J_{2}\left(P_{164}(\mathbb{C})\right)$ is studied and the $\alpha$ - and $\beta$-relations are written down explicitly.

Let $\iota^{!}: J_{p}\left(P_{n}(\mathbb{C})\right) \rightarrow J\left(L^{n}\left(p^{s}\right)\right)$ and put $\mathcal{J}(p, n, s ; k)=\iota^{!}(\mathcal{J}(p, n, k)),(k<$ $s)$. As a consequence of the $\alpha$-relations for the lens space, we obtain (e.g. Definition 7.3.4) that $\mathcal{J}(p, n, s ; k)=G\left(p,\left[n / p^{k}\right], s-k\right)$. From this and [17, Theorem 1.1] and [16, Theorem 1.2], we compute the order of $\psi_{\mathbb{R}}^{p^{k}}(\omega)$ in $J\left(L^{n}\left(p^{s}\right)\right)(k<s)$ (e.g. Proposition 7.3.7). Also the primary-decomposition of $J\left(L^{n}\left(p^{s}\right)\right)$ is written down when $n=p^{k}(p-1)+r,(0 \leqslant r \leqslant p-2)$ (e.g. Proposition 7.3.10).

The method of this paper, e.g. that of singular exponents and indices is new in literature. We believe that this method is absolutely indispensable to obtain the relations in the simple (computable) form that we have obtained. Our strategy is first to tackle the case of the complex projective space whose $\tilde{K}_{\mathbb{R}}$-groups are (almost) free by using the characteristic classes $\rho_{\mathbb{R}}^{k}$ where (as a result of freeness) the computations are straightforward and then to study the $J$-groups of lens spaces as extensions of quotient groups of those of complex projective spaces. In papers on $J$-groups of lens spaces, this approach via the complex projective space is missing. Direct assaults are made on lens spaces where their $\tilde{K}_{\mathbb{R}}$ groups are torsion groups and this makes the computations unnecessarily complicated and makes it impossible to obtain these relations in this simple (computable) form. Also, separate papers are devoted to the cases, $p$ odd and $p=2$, whereas in this paper, a unified treatment is given to cover both cases simultaneously.

Finally, these groups are looked on from a purely algebraic viewpoint in Section 8 , describing, briefly, how they naturally arise in algebra, drawing references to [13]. Let $D(\alpha, \beta ; n)$ be the finite Abelian group of equivalence classes of truncated weak $D$-series with rational coefficients defined in [13]. Let $D_{n}=D(\alpha, \beta ; n)$ for which $\alpha_{p}=p-1$ and $\beta_{p}=1$. Then one can let the Adams operation $\psi^{k}$ act on $D_{n}$ 
in a natural way. The subgroup of $D_{n}$ of truncated weak $D$-series which satisfy the Adams conjecture is, precisely, $J_{\mathbb{C}}\left(P_{n}(\mathbb{C})\right)$ which coincides with $J\left(P_{n}(\mathbb{C})\right)$ except for $n \equiv 1(\bmod 4)$ in which case, $J\left(P_{n}(\mathbb{C})\right)=J_{\mathbb{C}}\left(P_{n}(\mathbb{C})\right) \oplus \mathbb{Z}_{2}$. This motivates us to study the group $D_{n}$ (or, $D(\alpha, \beta ; n)$, in general) to which some of our methods may perhaps be adapted. Of course, the quotient group, $D_{n} / J_{\mathbb{C}}\left(P_{n}(\mathbb{C})\right)$ will measure how far these truncated weak $D$-series will deviate from satisfying the Adams conjecture. It is of interest to see whether the subgroup of $D(\alpha, \beta ; n)$ which satisfies the Adams conjecture carries any geometric significance for any $\alpha$ and $\beta$.

The present paper together with [14] constitute a self-contained solution to the problem. Reference [14] starts from scratch and determines the exact sequences relating the $J$-groups of lens spaces. In this present paper, technical references concerning $J\left(L^{n}\left(p^{k}\right)\right)$ are drawn mainly on [14].

Finally, the author would like to thank the referee for his suggestions.

\section{2. $J_{p}$-Order and the Characteristic Class $\rho_{\mathrm{R}}^{p}$}

\subsection{RELATION BETWEEN THE RATIONAL $J$-INVARIANT $\Omega$ AND THE CHARACTERISTIC CLASS $\rho_{\mathbb{R}}^{p}$}

Let for $k \in \mathbb{Z}^{+}, \mathbb{Q}$ and $\mathbb{Q}_{k}$ denote the rationals and the subring of rationals whose denominators are powers of $k$. Let $p$ be a prime and $\rho_{\mathbb{R}}^{p}: \tilde{K}_{\mathbb{R}}(X) \rightarrow 1+\tilde{K}_{\mathbb{R}}(X) \otimes \mathbb{Q}_{p}$ denote the characteristic class attached to the Adams operation $\psi_{\mathbb{R}}^{p}$ (e.g. [1, 2]; it is denoted by $\Theta_{p}$ in [7]). In [7, Section 16], a rational $J$-invariant $\Omega: \tilde{K}_{\mathbb{R}}(X) \rightarrow$ $1+\tilde{K}_{\mathbb{R}}(X) \otimes \mathbb{Q}$ is defined and it is proved in [7, Theorem 16.2] that if $x \in$ $\tilde{K}_{\mathbb{R}}(X)$ is $J$-trivial then $\Omega(x)$ is integral. In this section, we shall prove that $\Omega\left(\left(\psi_{\mathbb{R}}^{p}-1\right) x\right)=\rho_{\mathbb{R}}^{p}(x)$ in $1+\tilde{K}_{\mathbb{R}}(X) \otimes \mathbb{Q}$ which will enable us to prove a local converse to [7, Theorem 16.2] for bundles of the form $\left(\psi_{\mathbb{R}}^{p}-1\right) x, x \in \tilde{K}_{\mathbb{R}}(X)$.

PROPOSITION 2.1.1. Let $p$ be a prime and $\tilde{K}_{\mathbb{R}}(X)$ be free of $p$-torsion and $x \in \tilde{K}_{\mathbb{R}}(X)$. Then $J\left(\left(\psi_{\mathbb{R}}^{p}-1\right) x\right)=0$ if and only if $\rho_{\mathbb{R}}^{p}(x)$ is integral.

Proof. We have a commutative diagram,

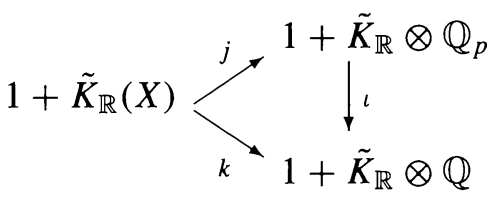

Since $\Omega$ is multiplicative and commutes with $\psi_{\mathbb{R}}^{p}$,

$$
\Omega\left(\left(\psi_{\mathbb{R}}^{p}-1\right) x\right)=\frac{\psi_{\mathbb{R}}^{p}(\Omega(x))}{\Omega(x)}=\rho_{\mathbb{R}}^{p}(x) \text { in } 1+\tilde{K}_{\mathbb{R}} \otimes \mathbb{Q}
$$

by $\left[7\right.$, Theorem 16.1], hence $\iota\left(\rho_{\mathbb{R}}^{p}(x)\right)=\Omega\left(\left(\psi_{\mathbb{R}}^{p}-1\right) x\right)$. Suppose that $J\left(\left(\psi_{\mathbb{R}}^{p}-1\right) x\right)=0$. Then it follows from [7, Theorem 16.2] that

$$
\begin{aligned}
\Omega\left(\left(\psi_{\mathbb{R}}^{p}-1\right) x\right) & =k(1+u), u \in \tilde{K}_{\mathbb{R}}(X) . \iota(j(1+u))=k(1+u) \\
& =\Omega\left(\left(\psi_{\mathbb{R}}^{p}-1\right) x\right)=\iota\left(\rho_{\mathbb{R}}^{p}(x)\right) .
\end{aligned}
$$


Let, for a prime $q, T_{q}(X)$ denote the $q$-torsion subgroup of $\tilde{K}_{\mathbb{R}}(X)$. Then $\rho_{\mathbb{R}}^{p}(x)$ and $j(1+u)$ differ by an element of ker

$$
\iota=\left(\oplus_{q \neq p} T_{q}(X) \otimes \mathbb{Q}_{p}\right)=\left(\oplus_{q \neq p} T_{q}(X)\right) \subset j\left(\tilde{K}_{\mathbb{R}}(X)\right),
$$

i.e.

$$
\rho_{\mathbb{R}}^{p}(x)=j(1+u)+j(v)=j(1+u+v), v \in \tilde{K}_{\mathbb{R}}(X) ;
$$

i.e. $\rho_{\mathbb{R}}^{p}(x)$ is integral.

Conversely, let $k \in \mathbb{Z}^{+}$. Then the co-cycle condition (cf. [1, Proposition 5.5], or, [7, Definition 8.1]) states that

$$
\rho_{\mathbb{R}}^{k p}(x)=\rho_{\mathbb{R}}^{k}(x) \psi_{\mathbb{R}}^{k}\left(\rho_{\mathbb{R}}^{p}(x)\right)=\rho_{\mathbb{R}}^{p}(x) \psi_{\mathbb{R}}^{p}\left(\rho_{\mathbb{R}}^{k}(x)\right) \quad \text { in } 1+\tilde{K}_{\mathbb{R}}(X) \otimes \mathbb{Q}_{k p} .
$$

Since the class $\rho_{\mathbb{R}}^{k}$ is multiplicative and commutes with the Adams operations,

$$
\rho_{\mathbb{R}}^{k}\left(\left(\psi_{\mathbb{R}}^{p}-1\right) x\right)=\frac{\rho_{\mathbb{R}}^{k}\left(\psi_{\mathbb{R}}^{p}(x)\right)}{\rho_{\mathbb{R}}^{k}(x)}=\frac{\psi_{\mathbb{R}}^{p}\left(\rho_{\mathbb{R}}^{k}(x)\right)}{\rho_{\mathbb{R}}^{k}(x)}=\frac{\psi_{\mathbb{R}}^{k}\left(\rho_{\mathbb{R}}^{p}(x)\right)}{\rho_{\mathbb{R}}^{p}(x)}
$$

in $1+\tilde{K}_{\mathbb{R}}(X) \otimes \mathbb{Q}_{k p}$.

The class $\rho_{\mathbb{R}}^{p}(x)$ is integral. Since $\tilde{K}_{\mathbb{R}}(X)$ is free of $p$-torsion, the inclusion-map, $\tilde{K}_{\mathbb{R}}(X) \otimes \mathbb{Q}_{k} \rightarrow \tilde{K}_{\mathbb{R}}(X) \otimes \mathbb{Q}_{k p}$ is 1-1. Hence, Equation (1.1) holds in $1+\tilde{K}_{\mathbb{R}}(X) \otimes$ $\mathbb{Q}_{k}$, i.e. $\rho_{\mathbb{R}}^{k}\left(\left(\psi_{\mathbb{R}}^{p}-1\right) x\right)=\psi_{\mathbb{R}}^{k}\left(\rho_{\mathbb{R}}^{p}(x)\right) / \rho_{\mathbb{R}}^{p}(x)$ in $1+\tilde{K}_{\mathbb{R}}(X) \otimes \mathbb{Q}_{k}$ It follows from the Adams conjecture (refer to [12] for an elementary proof and [2, Theorem 1.1]) that $J\left(\left(\psi_{\mathbb{R}}^{p}-1\right) x\right)=0$.

\section{2. $J_{p}$-TRIVIALITY AND INTEGRALITY OF THE CLASS $\rho_{\mathbb{R}}^{p}(x)$}

We know from [12] that the Adams operations pass to the quotient and act on the group $J(X)$. In this section, using [12, Proposition 2.3.3] to the effect that $\left(\psi_{\mathbb{R}}^{p}-1\right)$ is an isomorphism on $J_{p}(X)$ and is zero on $J_{q}(X)$ for $q \neq p$, we obtain from Proposition 2.1.1 that in the absence of $p$-torsion, a necessary and sufficient condition for $J_{p}$-triviality of $x \in \tilde{K}_{\mathbb{R}}(X)$ is integrality of the class $\rho_{\mathbb{R}}^{p}(x)$.

PROPOSITION 2.2.1. Let $p$ be a prime and $\tilde{K}_{\mathbb{R}}(X)$ be free of $p$-torsion. Then for $x \in \tilde{K}_{\mathbb{R}}(X), J_{p}(x)=0$ if and only if $\rho_{\mathbb{R}}^{p}(x)$ is integral.

Proof. Let $J(x)=u$ and $J_{p}(x)=u_{p}$. Since $\left(\psi_{\mathbb{R}}^{p}-1\right)=0$ on $J_{q}(x)$ for $q \neq p$ by [12, Proposition 2.3.3], it follows that $\left(\psi_{\mathbb{R}}^{p}-1\right) u=\left(\psi_{\mathbb{R}}^{p}-1\right) u_{p}$. Since $\left(\psi_{\mathbb{R}}^{p}-1\right)$ is an isomorphism on $J_{p}(X)$ by [12, Proposition 2.3.3], $u_{p}=0$ if and only if $\left(\psi_{\mathbb{R}}^{p}-1\right) u_{p}=0$ if and only if $\left(\psi_{\mathbb{R}}^{p}-1\right) u=0$ if and only if $\rho_{\mathbb{R}}^{p}(x)$ is integral by Proposition 2.1.1.

Remark 2.2.2. $\tilde{K}_{\mathbb{R}}\left(P_{n}(\mathbb{C})\right)$ is free of $p$-torsion except for $p=2$ and $n \equiv 1(\bmod 4)$ and Proposition 2.2.1 reduces the proof of the $J_{p}$-relations that we seek to checking whether the corresponding $\rho_{\mathbb{R}}^{p}$-classes are integral. 


\section{Coefficients of $\rho_{\mathbb{R}}^{p}\left(\psi_{\mathbb{R}}^{p^{k}}(\omega)\right)$ in $1+\tilde{K}_{\mathbb{R}}\left(P_{\infty}(\mathbb{C})\right) \otimes \mathbb{Q}_{p}$}

3.1. REVIEW OF $\tilde{K}_{\mathbb{R}}\left(P_{n}(\mathbb{C})\right)$

For $n \not \equiv 1(\bmod 4), \tilde{K}_{\mathbb{R}}\left(P_{n}(\mathbb{C})\right)$ is freely generated by $\left\{\omega, \omega^{2}, \ldots, \omega,{ }^{[n / 2]}\right\}$ subject to $\omega^{[n / 2]+1}=0$. For $n \equiv 1(\bmod 4), \tilde{K}_{\mathbb{R}}\left(P_{n}(\mathbb{C})\right)$ is generated by $\left\{\omega, \omega^{2}, \ldots\right.$, $\left.\omega^{[n / 2]+1}\right\}$ subject to the relation, $2 \omega^{[n / 2]+1}=\omega^{[n / 2]+2}=0$. The reader is referred to [4] for details.

3.2. ADAMS OPERATIONS AND THE ASSOCIATED CHARACTERISTIC CLASSES FOR THE COMPLEX PROJECTIVE SPACE

DEFINITION 3.2.1. Let $k \in \mathbb{Z}^{+}$. Define a power-series $T_{k}(x)$ with integer coefficients by

$$
\begin{aligned}
T_{k}(x) & =\sum_{q=1}^{k} \frac{k^{2}\left(k^{2}-1\right) \cdots\left(k^{2}-(q-1)^{2}\right)}{q^{2}\left(q^{2}-1\right) \cdots\left(q^{2}-(q-1)^{2}\right)} x^{q} \\
& =\sum_{q=1}^{k} \frac{k^{2}\left(k^{2}-1\right) \cdots\left(k^{2}-(q-1)^{2}\right)}{(1 / 2)(2 q) !} x^{q} .
\end{aligned}
$$

PROPOSITION 3.2.2. In $\tilde{K}_{\mathbb{R}}\left(P_{\infty}(\mathbb{C})\right), \psi_{\mathbb{R}}^{k}(\omega)=T_{k}(\omega)$.

Proof. This is [10, Theorem 5.2.4].

Let for a prime $p$ and a rational $q, v_{p}(q)$ denote the exponent of $p$ in the prime factorisation of $q$.

COROLLARY 3.2.3. Let $p$ be an odd prime. Then in $\tilde{K}_{\mathbb{R}}\left(P_{\infty}(\mathbb{C})\right)$,

$$
\psi_{\mathbb{R}}^{p}(\omega)=\sum_{i=1}^{p-1} a_{i} \omega^{i}+\omega^{p}
$$

where

$$
v_{p}\left(a_{i}\right)= \begin{cases}2, & 1 \leqslant i<\frac{1}{2}(p+1) \\ 1, & \frac{1}{2}(p+1) \leqslant i \leqslant p-1 .\end{cases}
$$

COROLLARY 3.2.4. Let $p$ be a prime. Then in $\tilde{K}_{\mathbb{R}}\left(P_{\infty}(\mathbb{C})\right)$,

$$
\psi_{\mathbb{R}}^{p}(\omega)=\sum_{i=1}^{p-1} a_{i} \omega^{i}+\omega^{p} \quad \text { where } a_{i} \equiv 0(\bmod p) \quad(1 \leqslant i \leqslant p-1) .
$$

PROPOSITION 3.2.5. Let $p$ be an odd prime and $k \in \mathbb{Z}^{+}$. Then in $\tilde{K}_{\mathbb{R}}\left(P_{\infty}(\mathbb{C})\right)$,

$$
\psi_{\mathbb{R}}^{p^{k}}(\omega)=\sum_{i=1}^{p^{k}-1} a_{i} \omega^{i}+\omega^{p^{k}}
$$


where

$$
\begin{aligned}
& a_{i} \equiv 0\left(\bmod p^{2}\right), \quad 1 \leqslant i<\frac{1}{2}(p+1) p^{k-1}, \\
& a_{i} \equiv 0(\bmod p), \quad \frac{1}{2}(p+1) p^{k-1} \leqslant i \leqslant p^{k}-1 .
\end{aligned}
$$

Proof. By induction on $k$. The case $k=1$ follows from Corollary 3.2.3. Let $k>1$ and assume the induction-hypothesis for $(k-1)$. By the induction-hypothesis, $\psi_{\mathbb{R}}^{p^{k-1}}(\omega)=\sum_{i=1}^{p^{k-1}-1} c_{i} \omega^{i}+\omega^{p^{k-1}}$, where $c_{i} \equiv 0\left(\bmod p^{2}\right), 1 \leqslant i<(1 / 2)(p+1) p^{k-2}$ and $c_{i} \equiv 0(\bmod p),(1 / 2)(p+1) p^{k-2} \leqslant i \leqslant p^{k-1}-1$. By Corollary 3.2.3, $\psi_{\mathbb{R}}^{p}(\omega)=\sum_{j=1}^{p-1} b_{j} \omega^{j}+\omega^{p}$, where $b_{j} \equiv 0\left(\bmod p^{2}\right), 1 \leqslant j<(1 / 2)(p+1)$ and $b_{j} \equiv 0$ $(\bmod p),(1 / 2)(p+1) \leqslant j \leqslant p-1$.

$$
\begin{aligned}
\psi_{\mathbb{R}}^{p^{k}}(\omega)= & \psi_{\mathbb{R}}^{p} \circ \psi_{\mathbb{R}}^{p^{k-1}}(\omega)=\sum_{i=1}^{p^{k-1}-1} c_{i}\left[\psi_{\mathbb{R}}^{p}(\omega)\right]^{i}+\left[\psi_{\mathbb{R}}^{p}(\omega)\right]^{p^{k-1}} \\
= & \sum_{i=1}^{p^{k-1}-1} c_{i}\left(b_{1} \omega+\cdots+b_{p-1} \omega^{p-1}+\omega^{p}\right)^{i}+ \\
& +\left(b_{1} \omega+\cdots+b_{p-1} \omega^{p-1}+\omega^{p}\right)^{p^{k-1}}
\end{aligned}
$$

$a_{i}=d_{i}+e_{i}$, where

$$
\begin{aligned}
& d_{i}=\sum_{\substack{j_{1}+j_{2}+\cdots+j_{p}=j \\
j_{1}+2 j_{2}+\cdots+p j_{p}=i}} \frac{j !}{j_{1} ! j_{2} ! \cdots j_{p} !} c_{j} b_{1}^{j_{1}} b_{2}^{j_{2}} \cdots b_{p-1}^{j_{p-1}}, \\
& e_{i}=\sum_{\substack{k_{1}+k_{2}+\cdots+k_{p}=p^{k-1} \\
k_{1}+2 k_{2}+\cdots+p k_{p}=i}} \frac{\left(p^{k-1}\right) !}{k_{1} ! k_{2} ! \cdots k_{p} !} b_{1}^{k_{1}} b_{2}^{k_{2}} \cdots b_{p-1}^{k_{p-1}} .
\end{aligned}
$$

Terms in the first sum for which $j_{s}>0$ for some $s<p$ contain $c_{j} \equiv 0(\bmod p)$ and $b_{s}^{j_{s}} \equiv 0(\bmod p)$ and, hence, congruent to $0\left(\bmod p^{2}\right)$. Terms for which $i_{s}=0$ $\forall s<p$ have $j=j_{p}$ and $i=p j_{p}$, i.e. $i=p j$. If $1 \leqslant i<(1 / 2)(p+1) p^{k-1}$ then $1 \leqslant j<(1 / 2)(p+1) p^{k-2}$ and $c_{j} \equiv 0\left(\bmod p^{2}\right)$ and this particular term is congruent to $0\left(\bmod p^{2}\right)$. Thus $d_{i} \equiv 0\left(\bmod p^{2}\right)$. If $(1 / 2)(p+1) p^{k-1} \leqslant i<p^{k-1}$, then $(1 / 2)(p+1) p^{k-2} \leqslant j<p^{k-2}$ and $c_{j} \equiv 0(\bmod p)$ and this particular term is congruent to $(\bmod p)$. Thus $d_{i} \equiv 0(\bmod p)$.

In the second sum, terms for which

$$
k_{1}+k_{2}+\cdots+k_{(p-1) / 2} \geqslant 1 \text { or } k_{(p+1) / 2}+\cdots+k_{p-1} \geqslant 2
$$

are congruent to $0\left(\bmod p^{2}\right)$. The particular term for which $k_{s}=1$ for $(p+1) / 2 \leqslant$ $s \leqslant p-1$ and $k_{s^{\prime}}=0 \forall 1 \leqslant s^{\prime} \leqslant p-1$ and $s^{\prime} \neq s$ is equal to $p^{k-1} b_{s} \equiv 0\left(\bmod p^{2}\right)$. Then $e_{i} \equiv 0\left(\bmod p^{2}\right)$. Thus, $a_{i} \equiv 0\left(\bmod p^{2}\right)$ if $1 \leqslant i<(1 / 2)(p+1) p^{k-1}$ and $a_{i} \equiv 0(\bmod p)$ if $(1 / 2)(p+1) p^{k-1} \leqslant i<p^{k-1}$. 
PROPOSITION 3.2.6. Let $k, q \in \mathbb{Z}^{+}$and

$$
u_{q}(k)=\left(k^{2}-1\right)\left(k^{2}-3^{2}\right) \cdots\left(k^{2}-(2 q-1)^{2}\right) .
$$

Then in $1+\tilde{K}_{\mathbb{R}}\left(P_{\infty}(\mathbb{C})\right) \otimes \mathbb{Q}_{k}$,

$$
\rho_{\mathbb{R}}^{k}(\omega)=1+\sum_{q=1}^{\infty} \frac{u_{q}(k)}{u_{q}(2 q+3)} \omega^{q}=1+\sum_{q=1}^{\infty} \frac{u_{q}(k)}{2^{2 q}(2 q+1) !} \omega^{q} .
$$

In particular,

(i) If $k$ is odd then $\rho_{\mathbb{R}}^{k}(\omega)$ is a polynomial of degree $(1 / 2)(k-1)$.

(ii) $\rho_{\mathbb{R}}^{2}(\omega)^{2}=\rho_{\mathbb{R}}^{2}(2 \omega)=1+(1 / 4) \omega$ and $\rho_{\mathbb{R}}^{2}\left(\psi_{\mathbb{R}}^{2}(\omega)\right)=1+(1 / 2) \omega$.

Proof. This is [10, Theorem 5.2.8].

COROLLARY 3.2.7. Let $p$ be an odd prime. Then in $1+\tilde{K}_{\mathbb{R}}\left(P_{\infty}(\mathbb{C})\right) \otimes \mathbb{Q}_{p}$, $\rho_{\mathbb{R}}^{p}(\omega)=1+\sum_{q=1}^{(1 / 2)(p-3)} a_{q} \omega^{q}+(1 / p) \omega^{(1 / 2)(p-1)}$ for $a_{q} \in \mathbb{Z}^{+} \quad(1 \leqslant q \leqslant(1 / 2)$ $(p-3))$.

PROPOSITION 3.2.8. Let $p$ be a prime and $k \in \mathbb{Z}$ such that $k \geqslant 0$ for $p$ odd and $k \geqslant 1$ for $p=2$. Then in $1+\tilde{K}_{\mathbb{R}}\left(P_{\infty}(\mathbb{C})\right) \otimes \mathbb{Q}_{p}$,

$$
\rho_{\mathbb{R}}^{p}\left(\psi_{\mathbb{R}}^{p^{k}}(\omega)\right)=1+\sum_{i=1}^{(1 / 2) p^{k}(p-1)-1} a_{i} \omega^{i}+\frac{1}{p} \omega^{(1 / 2) p^{k}(p-1)},
$$

where $a_{i} \in \mathbb{Z},\left(1 \leqslant i<(1 / 2)(p-1) p^{k}\right)$ and $a_{i} \equiv 0(\bmod p)$ for $1 \leqslant i \leqslant(p-1)$ $p^{k-1}-1$.

Proof.

(i) For $p=2$, it is proved by induction on $k$ starting with $k=1$ and by noting that $\rho_{\mathbb{R}}^{2}\left(\psi_{\mathbb{R}}^{2}(\omega)\right)=1+(1 / 2) \omega$ and $\psi_{\mathbb{R}}^{2}(\omega)=4 \omega+\omega^{2}$.

(ii) For $p=3, \rho_{\mathbb{R}}^{3}(\omega)=1+(1 / 3) \omega$ and $\rho_{\mathbb{R}}^{3}\left(\psi_{\mathbb{R}}^{3^{k}}(\omega)\right)=1+(1 / 3) \psi_{\mathbb{R}}^{3^{k}}(\omega)$ and it follows from Proposition 3.2.5. by noting that $(1 / 2)(p+1) p^{k-1}=(p-1) p^{k-1}$. (iii) For $p$ odd and $p \geqslant 5$,

$$
\rho_{\mathbb{R}}^{p}(\omega)=1+\sum_{i=1}^{(1 / 2)(p-1)} a_{i} \omega^{i}+(1 / p) \omega^{(1 / 2)(p-1)},(1 / 2)(p-1)>1 .
$$

Let $\psi_{\mathbb{R}}^{k}(\omega)=\sum_{j=1}^{p^{k}-1} b_{j} \omega^{j}+\omega^{p^{k}}$ where by Proposition $3.2 .5, b_{j} \equiv 0(\bmod p),(1 \leqslant$ $\left.j \leqslant p^{k}-1\right)$.

$$
\begin{aligned}
\rho_{\mathbb{R}}^{p}\left(\psi_{\mathbb{R}}^{p^{k}}(\omega)\right)= & 1+\sum_{i=1}^{1 / 2(p-1)} a_{i}\left(b_{1} \omega+\cdots+b_{p^{k}-1} \omega^{p^{k}-1}+\omega^{p^{k}}\right)^{i}+ \\
& +\frac{1}{p}\left(b_{1} \omega+\cdots+b_{p^{k}-1} \omega^{p^{k}-1}+p^{k}\right)^{(1 / 2)(p-1)}
\end{aligned}
$$


The products in the first sum whose coefficients are possibly not congruent to 0 $(\bmod p)$ are

$$
\underbrace{\omega^{p^{k}} \cdot \omega^{p^{k}} \cdots \omega^{p^{k}}}_{i}=\omega^{i p^{k}}
$$

and those in the second sum, are of the form $\omega^{j} \cdot \omega^{p^{k}} \cdots \omega^{p^{k}}=\omega^{j+(1 / 2)(p-3) p^{k}}$, $(j \geqslant 1)$ and both $i p^{k}, j+(1 / 2)(p-3) p^{k} \geqslant p^{k}>(p-1) p^{k-1}$.

\subsection{J-ORDER OF LINE BUNDLES}

In this section, we shall determine the $J$-order of line bundles over complex projective spaces and as a corollary, obtain a proof for Lam's result stated in [20] without proof. In what follows, let $M_{n}$ denote the Atiyah-Todd number of [5] defined by $M_{n}=\prod_{p} p^{v_{p}\left(M_{n}\right)}$, where

$$
v_{p}\left(M_{n}\right)=\sup _{1 \leqslant r \leqslant\left[\frac{n-1}{p-1}\right]}\left[r+v_{p}(r)\right]
$$

Let $M_{n, p}=p^{v_{p}\left(M_{n}\right)}$.

PROPOSITION 3.3.1. Let $n, k \in \mathbb{Z}^{+}$and $p$ be a prime. The order of $\psi_{\mathbb{R}}^{p^{k}}(\omega)$ in $J_{p}\left(P_{n}(\mathbb{C})\right)$ is $M_{\left[n / p^{k}\right]+1}, p$.

Proof. It follows from Proposition 3.2.8 that $\rho_{\mathbb{R}}^{p}\left(\psi_{\mathbb{R}}^{p^{k}}(\omega)\right)$ is a strict $D$-series of type $(\alpha, \beta)$ in the sense of [13, Definition 3.1] where

$$
\alpha_{p^{\prime}}= \begin{cases}\frac{1}{2} p^{i}(p-1) & \text { if } p^{\prime}=p \\ 1 & \text { if } p^{\prime} \neq p\end{cases}
$$

and $\beta_{p^{\prime}}=1$. As remarked in Section 3.1, for $n \not \equiv 1(\bmod 4), \tilde{K}_{\mathbb{R}}\left(P_{n}(\mathbb{C})\right)$ is torsion free and is freely generated by $\omega, \omega^{2}, \ldots, \omega^{[n / 2]}$ and we deduce from [11, Theorem 3.5] that the order of $\rho_{\mathbb{R}}^{p}\left(\psi_{\mathbb{R}}^{p^{k}}(\omega)\right)$ in $\left(1+\tilde{K}_{\mathbb{R}}\left(P_{n}(\mathbb{C})\right) \otimes \mathbb{Q}\right) /\left(1+\tilde{K}_{\mathbb{R}}\left(P_{n}(\mathbb{C})\right)\right)$ is $M_{\left[n / p^{k}\right]+1}, p$. By Proposition 2.2.1, this is the order of $\psi_{\mathbb{R}}^{p^{k}}(\omega)$ in $J_{p}\left(P_{n}(\mathbb{C})\right)$. For $n \equiv 1(\bmod 4)$ let $n=4 v+1$. By naturality, order of $\psi_{\mathbb{R}}^{p^{k}}(\omega)$ in $J_{p}\left(P_{4 v}(\mathbb{C})\right) \mid$ order of $\psi_{\mathbb{R}}^{p^{k}}(\omega)$ in $J_{p}\left(P_{4 v+1}(\mathbb{C})\right) \mid$ order of $\psi_{\mathbb{R}}^{p^{k}}(\omega)$ in $J_{p}\left(P_{4 v+2}(\mathbb{C})\right)$. By the torsion-free case, the first and the third orders are equal to $M_{\left[4 v / p^{k}\right]+1}, p$ and $M_{\left[4 v+2 / p^{k}\right]+1}$, $p$, respectively and $\left[4 v / p^{k}\right]=\left[4 v+1 / p^{k}\right]=\left[4 v+2 / p^{k}\right]$. Hence, the order of $\psi_{\mathbb{R}}^{p^{k}}(\omega)$ in $J_{p}\left(P_{4 v+1}(\mathbb{C})\right)$ is $M_{\left[4 v+1 / p^{k}\right]+1}, p$.

COROLLARY 3.3.2. Let $k=\prod_{i=1}^{s} p_{i}^{\alpha_{i}}$ for distinct primes $p_{i}$ and integers $\alpha_{i} \geqslant 1$. Then the order of $\psi_{\mathbb{R}}^{k}(\omega)$ in $J\left(P_{n}(\mathbb{C})\right)$ is $\prod_{i=1}^{s} M_{\left[n / p_{i}^{\alpha_{i}}\right]+1}, p_{i}$. 
Proof.

$$
\begin{aligned}
\psi_{\mathbb{R}}^{k}(\omega)= & \psi_{\mathbb{R}}^{p_{1}^{\alpha_{1}} \ldots p_{s}^{\alpha_{s}}}(\omega) \\
= & \left(\psi_{\mathbb{R}}^{p_{s}^{\alpha_{s}}}-1\right) \psi_{\mathbb{R}}^{p_{1}^{\alpha_{1}} \ldots p_{s-1}^{\alpha_{s-1}}}(\omega)+\left(\psi_{\mathbb{R}}^{p_{s-1}^{\alpha_{s-1}}}-1\right) \psi_{\mathbb{R}}^{p_{1}^{\alpha_{1}} \ldots p_{s-2}^{\alpha_{s-2}}}+\cdots+ \\
& +\left(\psi_{\mathbb{R}}^{p_{2}^{\alpha_{2}}}-1\right) \psi_{\mathbb{R}}^{p_{1}^{\alpha_{1}}}(\omega)+\psi_{\mathbb{R}}^{p_{1}^{\alpha_{1}}}(\omega) .
\end{aligned}
$$

It follows from the Adams conjecture that the terms on the right-hand side apart from the last one, e.g., $\psi_{\mathbb{R}}^{p_{1}^{\alpha_{1}}}(\omega)$, are of orders the powers of $p_{s}, p_{s-1}, \ldots, p_{2}$, respectively. Hence, the order of $\psi_{\mathbb{R}}^{k}(\omega)$ in $J_{p_{1}}\left(P_{n}(\mathbb{C})\right)$ is equal to the order of $\psi_{\mathbb{R}}^{p_{1}^{\alpha_{1}}}(\omega)$ in $J_{p_{1}}\left(P_{n}(\mathbb{C})\right)$ which by Proposition 3.3.1 is equal to $M_{\left[n / p_{1}^{\alpha_{1}}\right]+1}, p_{1}$. The same result is also true for the primes $p_{2}, \ldots, p_{s}$.

COROLLARY 3.3 .3 (Lam's result). Let $p$ be a prime and $n, k \in \mathbb{Z}^{+}$. Then $\psi_{\mathbb{R}}^{p^{k}}(\omega)$ is trivial in $J_{p}\left(P_{n}(\mathbb{C})\right)$ for $p^{k}>n /(p-1)$.

Proof. It follows from the definition of the Atiyah-Todd number that $M_{\left[n / p^{k}\right]+1}$, $p=1$ and the result follows from Proposition 3.3.1 .

\section{Singular Exponents and Indices}

\subsection{THE INTEGERS $t_{k}^{s}$}

Throughout, $n$ will denote a nonnegative integer and $p$ a fixed prime. For a real number $x$, let $[x]$ denote the greatest integer less than or equal to $x$. Let $r_{n}$ denote the greatest integer such that $p^{r_{n}} \leqslant n /(p-1)$.

DEFINITION 4.1.1. For $0 \leqslant s \leqslant r_{n}, 0 \leqslant k \leqslant r_{n}-s$, we define numbers $t_{k}^{s}$ inductively by $t_{0}^{s}=\left[n / p^{s}(p-1)\right]$ and having defined $t_{k-1}^{s}$, define $t_{k}^{s}$ by:

$$
t_{k}^{s}=\left[\frac{t_{k-1}^{s}-1}{p}\right]= \begin{cases}\frac{t_{k-1}^{s}}{p}-1 & \text { if } t_{k-1}^{s} \equiv 0(\bmod p), \\ {\left[\frac{t_{k-1}^{s}}{p}\right]} & \text { if } t_{k-1}^{s} \equiv 0(\bmod p),\end{cases}
$$

One can, easily, check that $t_{k}^{s}=\left[\left(n-p^{s}\left(p^{k}-1\right)\right) / p^{s+k}(p-1)\right]$. From now onwards we put $t_{k}=t_{k}^{0}=\left[\left(n-p^{k}+1\right) / p^{k}(p-1)\right]$. The numbers $\left\{t_{k}^{s}\right\}_{0 \leqslant k \leqslant r_{n}-s}$ form a strictly decreasing sequence.

LEMMA 4.1.2. Let $0 \leqslant i \leqslant j \leqslant k \leqslant r_{n}$. Then $t_{j}^{k-j} \leqslant t_{i}^{k-i}$.

Proof. We first prove the special case, $t_{1}^{k-1} \leqslant t_{0}^{k}$. Either

(i)

$$
t_{0}^{k-1}=\left[\frac{n}{p^{k-1}(p-1)}\right] \not \equiv 0(\bmod p)
$$


Then by definition,

Or,

$$
t_{1}^{k-1}=\left[\frac{1}{p}\left[\frac{n}{p^{k-1}(p-1)}\right]\right]=\left[\frac{n}{p^{k}(p-1)}\right]=t_{0}^{k} .
$$

(ii)

$$
t_{0}^{k-1}=\left[\frac{n}{p^{k-1}(p-1)}\right] \equiv 0(\bmod p) .
$$

Again by definition,

$$
t_{1}^{k-1}=\frac{1}{p}\left[\frac{n}{p^{k-1}(p-1)}\right]-1=\left[\frac{n}{p^{k}(p-1)}\right]-1=t_{0}^{k}-1<t_{0}^{k} .
$$

In the general case, let $i \leqslant h<h+1 \leqslant j$. It follows from the special case above that $t_{1}^{k-h-1} \leqslant t_{0}^{k-h} \cdot t_{h+1-i}^{k-h-1}$ and $t_{h-i}^{k-h}$ are obtained in the same inductive fashion from $t_{1}^{k-h-1}$ and $t_{0}^{k-h}$, respectively, in $(h-i)$-steps. Thus, $t_{h+1-i}^{k-h-1} \leqslant t_{h-i}^{k-h}$. Hence, $t_{j-i}^{k-j} \leqslant t_{j-i-1}^{k-j+1} \leqslant t_{j-i-2}^{k-j+2} \leqslant \cdots \leqslant t_{0}^{k-i}$.

$t_{j}^{k-j}$ and $t_{i}^{k-i}$ are obtained in the same inductive fashion from $t_{j-i}^{k-j}$ and $t_{0}^{k-i}$ in $i$-steps. Hence, $t_{j}^{k-j} \leqslant t_{i}^{k-i}$.

DEFINITION 4.1.3. For $0 \leqslant s \leqslant r_{n}$, define $\Phi_{n, s}=\bigcup_{k=0}^{r_{n}-s}\left\{t_{k}^{s}\right\}$.

$$
\Phi_{n, s}^{0}=\left\{t_{k}^{s} \in \Phi_{n, s}\right\} t_{k}^{s} \neq 0 \quad \text { and } \quad t_{k}^{s} \equiv 0 \quad(\bmod p) .
$$

Put $\Phi_{n}=\Phi_{n, 0}$ and $\Phi_{n}^{0}=\Phi_{n, 0}^{0}$.

OBSERVATION 4.1.4. If $k$ is the least integer such that $t_{k} \in \Phi_{n}^{0}$ and $0 \leqslant s \leqslant k \leqslant r_{n}$ then

$$
t_{j}^{s}=t_{s+j}=\left[\frac{n}{p^{s+j}(p-1)}\right] \quad(0 \leqslant j \leqslant k-s) .
$$

COROLLARY 4.1.5. If $\Phi_{n}^{0}=\phi$, then $\Phi_{n, s}^{0}=\phi\left(0 \leqslant s \leqslant r_{n}\right)$ and $t_{j}^{s}=t_{s+j}$ $\left(0 \leqslant j \leqslant r_{n}-s\right)$.

OBSERVATION 4.1.6. Let $0 \leqslant s \leqslant r_{n}, 0 \leqslant j<i \leqslant r_{n}-s$, then

$$
p^{i-j} t_{i}^{s}+\frac{p^{i-j}-1}{p-1} \leqslant t_{j}^{s} \leqslant p^{i-j} t_{i}^{s}+\frac{p\left(p^{i-j}-1\right)}{p-1}
$$

with equality attained on the left-hand side if and only if $t_{k}^{s}=p t_{k+1}^{s}+1, \forall j \leqslant k<i$ and on the right-hand side if and only if $t_{k}^{s}=p t_{k+1}^{s}+p, \forall j \leqslant k<i$. If further $t_{k}^{s} \notin \Phi_{n, s}^{0}, \forall j \leqslant k<i$, then $t_{j}^{s} \leqslant p^{i-j} t_{i}^{s}+p^{i-j}-1$ with equality attained if and only if $t_{k}^{s}=p t_{k+1}^{s}+p-1, \forall j \leqslant k<i$.

Proof. The first part follows by induction from the inequality, $p t_{i}^{s}+1 \leqslant t_{i-1}^{s} \leqslant$ $p t_{i}^{s}+p$ and the second part from the inequality, $t_{i-1}^{s} \leqslant p t_{i}^{s}+p-1$. 
COROLLARY 4.1.7. Let $0 \leqslant s \leqslant r_{n}, 0 \leqslant j<i \leqslant r_{n}-s$. Then $p^{i-j} t_{i}^{s}+i-j \leqslant t_{j}^{s}$.

COROLLARY 4.1.8. Let $s, i \in \mathbb{Z}^{+}$. Then

$$
p^{s+i}(p-1) t_{i}^{s}+p^{s}\left(p^{i}-1\right) \leqslant n \leqslant p^{s+i}(p-1) t_{i}^{s}+p^{s+1}\left(p^{i}-1\right) .
$$

If further $t_{k}^{s} \notin \Phi_{n, s}^{0} \forall 0 \leqslant k<i$ then

$$
n \leqslant p^{s+i}(p-1) t_{i}^{s}+p^{s}(p-1)\left(p^{i}-1\right) .
$$

Proof. Put $j=0$ in Observation 4.1.6 and note that $t_{0}^{s}=\left[n / p^{s}(p-1)\right]$.

DEFINITION 4.1.9. Let $0 \leqslant s \leqslant r_{n}, 0 \leqslant k \leqslant r_{n}-s$ and for $t_{k}^{s} \in \Phi_{n, s}^{0}$, let $t_{k}^{s}=$ $p^{v} \triangle,(v \geqslant 1,(\triangle, p)=1)$. Then it follows from Definition 4.1.1 that $t_{j}^{s}=p^{k+v-j}$ $\Delta-1(k+1 \leqslant j \leqslant k+v)$. Define

$$
T_{k}^{s}=\bigcup_{j=k}^{k+v}\left\{t_{j}^{s}\right\}=\left\{p^{\nu} \Delta\right\} \cup \bigcup_{j=k+1}^{k+\nu}\left\{p^{k+\nu-j} \Delta-1\right\} .
$$

Let $T_{k}=T_{k}^{0}$.

OBSERVATION 4.1.10. Let $0 \leqslant s \leqslant r_{n}, 0 \leqslant k, l \leqslant r_{n}-s$. Then for distinct $t_{k}^{s}, t_{l}^{s} \in$ $\Phi_{n, s}^{0}$, the sets $T_{k}^{s}-\left\{t_{k}^{s}\right\}$ and $T_{l}^{s}-\left\{t_{l}^{s}\right\}$ are disjoint. If for $k<l, t_{k}^{s}, t_{l}^{s}$ are consecutive in $\Phi_{n, s}^{0}$ then $T_{k}^{s} \cap T_{l}^{s}$ is either empty or $\left\{t_{l}^{s}\right\}$ and the latter is always the case for $p=2$.

DEFINITION 4.1.11. Let $0 \leqslant s \leqslant r_{n}, 0 \leqslant k \leqslant r_{n}-s$. Define $\Phi_{n, s}^{\prime}=\bigcup_{t_{k}^{s} \in \Phi_{n, s}^{0}}\left(T_{k}^{s}-\right.$ $\left.\left\{t_{k}^{s}\right\}\right)$ and $\Phi_{n, s}^{\prime \prime}=\Phi_{n, s}-\Phi_{n, s}^{\prime}$.

LEMMA 4.1.12. Let $0 \leqslant s \leqslant r_{n}, 0 \leqslant k \leqslant r_{n}-s$. Then

$$
t_{k}^{s}= \begin{cases}{\left[\frac{n}{p^{s+k}(p-1)}\right]-1} & \text { if } t_{k}^{s} \in \Phi_{n, s}^{\prime}, \\ {\left[\frac{n}{p^{s+k}(p-1)}\right]} & \text { if } t_{k}^{s} \in \Phi_{n, s}^{\prime \prime} .\end{cases}
$$

Proof. It is by induction on $k$. For $k=0$, it follows from Definition 4.1.11 and the fact that $t_{0}^{s} \in \Phi_{n, s}^{\prime \prime}$. Let $k>1$ and assume the induction-hypothesis for all $k^{\prime}<k$. Suppose $t_{k}^{s} \in \Phi_{n, s}^{\prime}$, i.e. that $t_{k}^{s} \in T_{i}^{s}-\left\{t_{i}^{s}\right\}$ for $t_{i}^{s} \in \Phi_{n, s}^{0}$. Let $t_{i}^{s}=$ $p^{v} \Delta,(v \geqslant 1,(\Delta, p)=1)$. Then $k \leqslant v+i$ and $t_{k}^{s}=p^{\nu-k+i} \Delta-1$. According to the induction-hypothesis,

$$
t_{i}^{s}=\left[\frac{n}{p^{s+i}(p-i)}\right]-\epsilon,
$$

where

$$
\epsilon= \begin{cases}1 & \text { if } t_{i}^{s} \in \Phi_{n, s}^{\prime}, \\ 0 & \text { if } t_{i}^{s} \in \Phi_{n, s}^{\prime \prime}\end{cases}
$$




$$
\begin{aligned}
t_{k}^{s} & =\frac{p^{v} \Delta}{p^{k-i}}-1=\frac{\left[n / p^{s+i}(p-1)\right]-\epsilon}{p^{k-i}}-1=\left[\frac{\left[n / p^{s+k}(p-i)\right]}{p^{k-i}}\right]-1 \\
& =\left[\frac{n}{p^{s+i}(p-i)}\right]-1 .
\end{aligned}
$$

Suppose $t_{k}^{s} \in \Phi_{n, s}^{\prime \prime}$. Then $t_{k-1}^{s} \notin \Phi_{n, s}^{0}$ and $t_{k}^{s}=\left[t_{k-1}^{s} / p\right]$. By the induction hypothesis,

$$
t_{k-1}^{s}=\left[\frac{n}{p^{s+k-1}(p-1)}\right]-\epsilon,
$$

where

$$
\epsilon= \begin{cases}1 & \text { if } t_{k-1}^{s} \in \Phi_{n, s}^{\prime}, \\ 0 & \text { if } t_{k-1}^{s} \in \Phi_{n, s}^{\prime \prime} .\end{cases}
$$

If $t_{k-1}^{s} \in \Phi_{n, s}^{\prime \prime}$ then

$$
t_{k}^{s}=\left[\frac{\left[n / p^{s+k-1}(p-1)\right]}{p}\right]=\left[\frac{n}{p^{s+k}(p-1)}\right] .
$$

If $t_{k-1}^{s} \in \Phi_{n, s}^{\prime}$, then let $t_{k-1}^{s} \in T_{i}^{s}-\left\{t_{i}^{s}\right\}$ for some $t_{i}^{s} \in \Phi_{n, s}^{0}$. Suppose that $t_{i}^{s}=$ $p^{v} \triangle(v \geqslant 1,(\triangle, p)=1)$. Since $t_{k}^{s} \notin \Phi_{n, s}^{\prime}, k-1=i+v$ and $t_{k-1}^{s}=\Delta-1$, $\left(t_{k-1}^{s}, p\right)=\left(t_{k-1}^{s}+1, p\right)=1$ and, thus,

$$
t_{k}^{s}=\left[\frac{t_{k-1}^{s}}{p}\right]=\left[\frac{t_{k-1}^{s}+1}{p}\right]=\left[\frac{\left[n / p^{s+k-1}(p-1)\right]}{p}\right]=\left[\frac{n}{p^{s+k}(p-1)}\right] .
$$

COROLLARY 4.1.13. Let $p=2,0 \leqslant s \leqslant r_{n}, \Phi_{n, s}^{0} \neq \emptyset$ and $k_{0}$, is the smallest integer such that $t_{k_{0}}^{s} \in \Phi_{n, s}^{0}$. If $k>k_{0}$, then $t_{k}^{s}=\left[n / 2^{s+k}\right]-1$.

Proof. If $t_{k}^{s} \in \Phi_{n, s}^{0}, t_{k}^{s}=2^{v} \Delta(v \geqslant 1, \Delta$ odd $)$ then the last element of $T_{k}^{s}$ is $t_{k+\nu}^{s}=\Delta-1$. If $\Delta \neq 1$ then $t_{k+\nu}^{s} \in \Phi_{n, s}^{0}$ and the sets $T_{k}^{s}-\left(t_{k}^{s}\right)$ and $T_{k+\nu}^{s}-\left(t_{k+\nu}^{s}\right)$ are adjacent. Hence, by definition, $\Phi_{n, s}^{\prime}=\bigcup_{k>k_{0}} t_{k}^{s}$ and the result follows from Lemma 4.1.12.

COROLLARY 4.1.14. Let $p=2,0 \leqslant s \leqslant r_{n}$ and $\Phi_{n, s}^{0} \neq \emptyset$. Then $k$ is the largest integer such that $t_{k}^{s} \in \Phi_{n, s}^{0}$, if and only if $t_{k}^{s}=2^{v}$ for $v \geqslant 1$ (i.e. $\Delta=1$ ).

Proof. Let $t_{k}^{s}=2^{v} \Delta \in \Phi_{n, s}^{0}\left(v \geqslant 1, \Delta\right.$ odd). Then $t_{k+v}^{s}=\Delta-1$ is even; i.e., either, $t_{k+\nu}^{s}=0$, or, $t_{k+\nu}^{s} \in \Phi_{n, s}^{0}$. Hence, $k$ is the largest integer such that $t_{k}^{s} \in \Phi_{n, s}^{0}$ if and only if $t_{k+v}^{s}=0$ if and only if $\Delta=1$.

\subsection{THE COEFFICIENT $b_{m}^{s, j}$ AND THE ASSOCIATED SEQUENCES}

DEFINITION 4.2.1. Let $0 \leqslant s \leqslant r_{n}, 0 \leqslant j \leqslant r_{n}-s(s, j) \neq(0,0)$ if $p=2, q=$ $(1 / 2)(p-1) p^{s+j}$. Let

$$
\rho_{\mathbb{R}}^{p}\left(\psi_{\mathbb{R}}^{p^{s+j}}(\omega)\right)=1+\sum_{k=1}^{q-1} a_{k} \omega^{k}+\frac{1}{p} \omega^{q} .
$$




$$
\rho_{\mathbb{R}}^{p}\left(\psi_{\mathbb{R}}^{p^{s+j}}(\omega)\right)^{p^{t_{j}^{s}}}=1+\sum_{m=1}^{[n / 2]} b_{m}^{s, j} \omega^{m} .
$$

Define for $1 \leqslant m \leqslant[n / 2]$,

$$
\begin{aligned}
S_{m}^{s, j} & =\left\{s=\left(s_{0}, s_{1}, \ldots, s_{q}\right): s_{0}+s_{1}+\cdots+s_{q}\right. \\
& \left.=p^{t_{j}^{s}}, s_{1}+2 s_{2}+\cdots+q s_{q}=m\right\} .
\end{aligned}
$$

For $s \in S_{m}^{s, j}$,

$$
T(s)=\frac{\left(p^{t_{j}^{s}}\right) !}{s_{0} ! s_{1} ! \cdots s_{q} !} a_{1}^{s_{1}} a_{2}^{s_{2}} \cdots a_{q-1}^{s_{q-1}}\left(\frac{1}{p}\right)^{s_{q}} .
$$

Then $b_{m}^{s, j}=\sum_{s \in S_{m}^{s, j}} T(s)$.

DEFINITION 4.2.2. Let $0 \leqslant s \leqslant r_{n}, 0 \leqslant j \leqslant r_{n}-s$. For $m \equiv 0(\bmod q)$, i.e. $m \equiv$ $0\left(\bmod \left(1 / 2(p-1) p^{s+j}\right)\right)$, let $s^{0}(s, j, m)$ be the unique sequence defined by $s_{1}=\cdots=s_{q-1}=0, s_{q}=m / q, s_{0}=p^{t_{j}^{s}}-m / q$. When there is no ambiguity, we shall, simply, write $s^{0}$ instead of $s^{0}(s, j, m)$. If we express $m=(1 / 2)(p-1)$ $p^{s+i} \Delta,(j \leqslant i,(\Delta, p)=1)$ then $s_{1}^{0}=\cdots=s_{q-1}^{0}=0, s_{q}^{0}=p^{i-j} \Delta, s_{0}^{0}=$ $p^{t_{j}^{s}}-p^{i-j} \Delta$. Then

$$
T\left(s^{0}\right)=\left(\begin{array}{c}
p^{t_{j}^{s}} \\
p^{i-j} \Delta
\end{array}\right)\left(\frac{1}{p}\right)^{p^{i-j} \Delta}
$$

If $p=2$ and $(s, j)=(0,0)$ assume without loss of generality that $t_{0}=n$ is even. Then $\rho_{\mathbb{R}}^{2}(\omega)^{2^{t_{0}}}=\left[\rho_{\mathbb{R}}^{2}(\omega)^{2}\right]^{t^{t_{0}-1}}$. For $1 \leqslant m \leqslant n / 2$, define $s^{0}(0,0, m)$ by $s_{1}=m, s_{0}=2^{t_{0}}-m$ and

$$
T\left(s^{0}\right)=\left(\begin{array}{c}
2^{t_{0}-1} \\
m
\end{array}\right)\left(\frac{1}{4}\right)^{m}=\left(\begin{array}{c}
2^{t_{0}-1} \\
m
\end{array}\right) \frac{1}{2^{2 m}} .
$$

In this case, $b_{m}^{0,0}=T\left(s^{0}\right)$.

DEFINITION 4.2.3. Let $0 \leqslant s \leqslant r_{n}, i \geqslant 1,0 \leqslant j<i$ and $m=(1 / 2)(p-1) p^{s+i} \triangle$, $2 m \leqslant n,(\triangle, p)=1$. Define the $(s, j)$-index of $m$ by $\varepsilon_{m}^{s, j}=p^{i-j} \Delta+i-j-t_{j}^{s}$. Let $\varepsilon_{m}^{j}=\varepsilon_{m}^{0, j}$.

LEMMA 4.2.4. $v_{p}\left(T\left(s^{0}\right)\right)=-\varepsilon_{m}^{s, j}$.

Proof. It follows from [14, Lemma 2.2.10] that

$$
v_{p}\left(\left(\begin{array}{c}
p^{t_{j}^{s}} \\
p^{i-j} \Delta
\end{array}\right)\right)=t_{j}^{s}-(i-j)
$$

and hence $v_{p}\left(T\left(s^{0}\right)\right)=t_{j}^{s}-(i-j)-p^{i-j} \Delta=-\varepsilon_{m}^{s, j}$ except for $p=2$ and $(s, j)=(0,0)$, in which case, $v_{2}\left(T\left(s^{0}\right)\right)=t_{0}-1-v_{2}(m)-2 m$ by [14, Lemma 
2.2.10]. If $m=(1 / 2) 2^{i} \Delta=2^{i-1} \Delta(i \geqslant 1, \Delta$ odd $)$, then

$$
v_{2}\left(T\left(s^{0}\right)\right)=t_{0}-1-(i-1)-2^{i} \Delta=-\left(2^{i} \Delta+i-t_{0}\right)=-\varepsilon_{m}^{0,0} .
$$

\subsection{INTEGRALITY THEOREMS ON $T(s)$}

Let $0 \leqslant s \leqslant r_{n}, 0 \leqslant j, j^{\prime} \leqslant r_{n}-s, 1 \leqslant m, m^{\prime} \leqslant[n / 2]$. In this section, we shall prove that for $s \in S_{m}^{s, j}, s \neq s^{0}(s, j, m), T(s), T(s) T\left(s^{0}(s, j, m)\right)$ and $T\left(s^{0}(s, j, m)\right)$ $T\left(s^{0}\left(s, j^{\prime}, m^{\prime}\right)\right)$ are all integral if $(j, m) \neq\left(j^{\prime}, m^{\prime}\right)$.

LEMMA 4.3.1. Let $0 \leqslant s \leqslant r_{n}, 0 \leqslant j \leqslant r_{n}-s$ and $1 \leqslant m \leqslant[n / 2]$. If $s \in S_{m}^{s, j}-$ $\left\{s^{0}(s, j, m)\right\}$ then $T(s) \in \mathbb{Z}$.

Proof. Let $\rho_{\mathbb{R}}^{p}\left(\psi_{\mathbb{R}}^{p^{s+j}}(\omega)\right)=1+\sum_{i \geqslant 1} a_{i} \omega^{i}$ as in Proposition 3.2.8. Let $q=$ $(1 / 2)(p-1) p^{s+j}$. Since $s \neq s^{0}, s_{i}>0$ for some $1 \leqslant i \leqslant q-1 . q s_{q} \leqslant m$ and thus $s_{q} \leqslant[m / q]$. Let $\beta=[m / q]-s_{q} \geqslant 0$. We consider the following four cases:

Case (i): $s_{i}>0$ for $i<(p-1) p^{s+j-1}$;

Case (ii): $s_{i}>0$ for $i \geqslant(p-1) p^{s+j-1}$.

Case (ii-a): $\beta \geqslant 1$.

Case (ii-b): $t_{j}^{s} \in \Phi_{n, s}^{\prime \prime}$.

Case (ii-c): $\beta=0$ and $t_{j}^{s} \in \Phi_{n, s}^{\prime}$.

We number these four cases as 1, 2, 3, 4 and define

$$
\varepsilon_{h}= \begin{cases}1 & \text { for } h=1,2, \text { i.e. in cases (i) and (ii-a), } \\ 0 & \text { for } h=3,4, \text { i.e. in cases (ii-b) and (ii-c). }\end{cases}
$$

$\theta_{h}(s)=[m / q]-\varepsilon_{h}-v_{p}\left(s_{i}\right)-s_{q}+v_{p}\left(a_{1}^{s_{1}} \cdots a_{q-1}^{s_{q-1}}\right)$. We shall prove that in case $h, v_{p}(T(s)) \geqslant \theta_{h}(s) \geqslant 0,(1 \leqslant h \leqslant 4)$.

$$
T_{s}=\frac{\left(p^{t_{j}^{s}}\right) !}{s_{0} ! s_{1} ! \cdots s_{q} !}\left(\frac{1}{p}\right)^{s_{q}}\left(a_{1}^{s_{1}} \cdots a_{q-1}^{s_{q-1}}\right) .
$$

By [5, Lemma 6.2],

$$
v_{p}\left(\frac{\left(p^{t_{j}^{s}}\right) !}{s_{0} ! s_{1} ! \cdots s_{q} !}\right) \geqslant t_{j}^{s}-\inf _{0 \leqslant j \leqslant q} v_{p}\left(s_{j}\right) \geqslant t_{j}^{s}-v_{p}\left(s_{i}\right)
$$

and by Lemma 4.1.12, $t_{j}^{s} \geqslant\left[n / p^{s+j}(p-1)\right]-\varepsilon_{h}$. Since

$$
n \geqslant 2 m, t_{j}^{s} \geqslant[m / q]-\varepsilon_{h},(1 \leqslant h \leqslant 3) .
$$

Hence,

$$
v_{p}(T(s)) \geqslant[m / q]-\varepsilon_{h}-v_{p}\left(s_{i}\right)-s_{q}+v_{p}\left(a_{1}^{s_{1}} \cdots a_{q-1}^{s_{q-1}}\right)=\theta_{h}(s),(1 \leqslant h \leqslant 3) .
$$


For $h=1$, i.e. in case (i), it follows from Proposition 3.2.8 that $v_{p}\left(a_{1}^{s_{1}} \cdots a_{q-1}^{s_{q-1}}\right)$ $\geqslant s_{i} . \theta_{1}(s) \geqslant\left([m / q]-s_{q}\right)+\left(s_{i}-v_{p}\left(s_{i}\right)-1\right) \geqslant 0$.

For $h=2, p^{s+j-1}(p-1) s_{i} \leqslant s_{1}+\cdots+i s_{i}+\cdots+(q-1) s_{q-1}=m-q s_{q}=$ $\beta q+r,(0 \leqslant r<q)$.

$$
s_{i} \leqslant \frac{\beta}{2} p+\frac{r}{p^{s+j-1}(p-1)}<\left(\frac{\beta+1}{2}\right) p \leqslant p^{\beta},
$$

i.e. $s_{i}<p^{\beta}$ and $v_{p}\left(s_{i}\right)<\beta-1 . \theta_{2}(s)=[m / q]-s_{q}-1-v_{p}\left(s_{i}\right)=\beta-1-v_{p}\left(s_{i}\right) \geqslant 0$ by the above.

For $h=3$, as in the proof of the case for $h=2$ above, $\theta_{3}(s)=[m / q]-s_{q}-$ $v_{p}\left(s_{i}\right)=\beta-v_{p}\left(s_{i}\right) \geqslant 0$. This completes the proof for the first three cases.

For $h=4, t_{j}^{s} \in \Phi_{n, s}^{\prime}$. Let $t_{j}^{s} \in T_{k}^{s}-\left\{t_{k}^{s}\right\}$ for some $t_{k}^{s}=p^{v} \Delta \in \Phi_{n, s}^{0}(v \geqslant 1$, $(\Delta, p)=1) . m \geqslant q s_{q}+i s_{i} \geqslant q s_{q}+p^{s+j-1}(p-1)$.

$$
n \geqslant 2 m \geqslant p^{s+j}(p-1) s_{q}+2 p^{s+j-1}(p-1) .
$$

Also, $p^{v} \Delta=t_{k}^{s} \geqslant\left[n / p^{s+k}(p-1)\right]-1$ by Lemma 4.1.12, i.e.

$$
\begin{aligned}
& n \leqslant p^{s+k+\nu}(p-1) \Delta+p^{s+k}(p-1)+r,\left(0 \leqslant r<p^{s+k}(p-1)\right), \text { i.e., } \\
& n<p^{s+k+\nu}(p-1) \Delta+2 p^{s+k}(p-1) .
\end{aligned}
$$

We deduce from inequalities (4.1) and (4.2) that $s_{q}<p^{\nu+j-k} \Delta=t_{j}^{s}+1$ or, equivalently, that $[m / q]=s_{q} \leqslant t_{j}^{s}$. Since $t_{j}^{s} \geqslant[m / q]$,

$$
\begin{aligned}
v_{p}(T(s)) & \geqslant t_{j}^{s}-v_{p}\left(s_{i}\right)-s_{q}+v_{p}\left(a_{1}^{s_{1}} \cdots a_{q-1}^{s_{q-1}}\right) \\
& \geqslant\left[\frac{m}{q}\right]-v_{p}\left(s_{i}\right)-s_{q}+v_{p}\left(a_{1}^{s_{1}} \cdots a_{q-1}^{s_{q-1}}\right)=\theta_{4}(s) .
\end{aligned}
$$

$s_{q}=[m / q]$ and as in the proof of $h=2, s_{i}<p / 2$ and thus $v_{p}\left(s_{i}\right)=0$. Hence $\theta_{4}(s) \geqslant 0$.

Corollary 4.3.2. Let $0 \leqslant s \leqslant r_{n}, 0 \leqslant j \leqslant r_{n}-s$ and $1 \leqslant m \leqslant[n / 2]$. If $m \not \equiv 0(\bmod$ $\left.(1 / 2)(p-1) p^{s+j}\right)$ then $b_{m}^{s, j} \in \mathbb{Z}$.

Proof. $b_{m}^{s, j}=\sum_{s \in S_{m}^{s, j}} T(s)$ and since $m \not \equiv 0\left(\bmod \left((1 / 2)(p-1) p^{s+j}\right)\right.$, the distinguished sequence $s^{0}(s, j, m)$ is not defined and thus $T(s) \in \mathbb{Z}, \forall s \in S_{m}^{s, j}$ by Lemma 4.3.1.

COROLLARY 4.3.3. Let $0 \leqslant s \leqslant r_{n}, i \geqslant 1$ and $m=(1 / 2)(p-1) p^{s+i} \Delta(1 \leqslant m \leqslant$ $[n / 2],(\Delta, p)=1)$. Then for $0 \leqslant j \leqslant i, b_{m}^{s, j} \notin \mathbb{Z}$ if and only if $\varepsilon_{m}^{s, j}>0$ in which case, $v_{p}\left(b_{m}^{s, j}\right)=-\varepsilon_{m}^{s, j}$.

Proof. $b_{m}^{s, j}=T\left(s^{0}\right)+\sum_{s \neq s^{0}} T(s)$. The second term on the right-hand side is integral by Lemma 4.3.1 and $v_{p}\left(T\left(s^{0}\right)\right)=-\varepsilon_{m}^{s, j}$ by Lemma 4.2.4. 
LEMMA 4.3.4. Let $0 \leqslant s \leqslant r_{n}, 0 \leqslant j \neq j_{1} \leqslant r_{n}-s, 0 \leqslant j_{1} \leqslant i_{1}, i_{1} \geqslant 1$ and $m_{1}=$ $(1 / 2)(p-1) p^{s+i_{1}} \triangle_{1},\left(2 m_{1} \leqslant n,\left(\triangle_{1}, p\right)=1\right), s \in S_{m}^{s, j}(2 m \leqslant n)$ not be a distinguished sequence (i.e. $s \neq s^{0}(s, j, m)$ ) and $s^{0}=s^{0}\left(s, j_{1}, m_{1}\right)$ be a distinguished sequence. If $2 m+2 m_{1} \leqslant n$ then $T(s) T\left(s^{0}\right) \in \mathbb{Z}$.

Proof. Let $q=(1 / 2)(p-1) p^{s+j}, q_{1}=(1 / 2)(p-1) p^{s+j_{1}}$ and $\theta_{h}(s)$ be as defined in case $h,(1 \leqslant h \leqslant 4)$, as in the proof of Lemma 4.3.1. We shall prove that in case $h, v_{p}(T(s))+v_{p}\left(T\left(s^{0}\right)\right) \geqslant \theta_{h}(s)+\left[m_{1} / q\right]+\left[m / q_{1}\right]-\left(i_{1}-j_{1}+1\right)$ and that $\left[m_{1} / q\right]+\left[m / q_{1}\right]-\left(i_{1}-j_{1}+1\right) \geqslant 0$. The result will follow from the inequality $\theta_{h}(s) \geqslant 0$ proved in the proof of Lemma 4.3.1. As in the proof of Lemma 4.3.1, $v_{p}(T(s)) \geqslant t_{j}^{s}-\inf _{j} v_{p}\left(s_{j}\right)-s_{q}+v_{p}\left(a_{1}^{s_{1}} \cdots a_{q-1}^{s_{q-1}}\right)$ and by Lemma 4.2.4, $v_{p}\left(T\left(s^{0}\right)\right)=t_{j_{1}}^{s}-p^{i_{1}-j_{1}} \triangle_{1}-\left(i_{1}-j_{1}\right) \geqslant\left[n / p^{s+j_{1}}(p-1)\right]-1-p^{i_{1}-j_{1}} \triangle_{1}-\left(i_{1}-j_{1}\right)$ by Lemma 4.1 .12 and since $n \geqslant 2 m+2 m_{1}$,

$$
\begin{aligned}
v_{p}\left(T\left(s^{0}\right)\right)= & {\left[\frac{m}{(1 / 2)(p-1) p^{s+j_{1}}}\right]+\left[\frac{m_{1}}{(1 / 2)(p-1) p^{s+j_{1}}}\right]-} \\
& -1-p^{i_{1}-j_{1}} \triangle_{1}-\left(i_{1}-j_{1}\right) \\
= & {\left[\frac{m}{q_{1}}\right]+\left(\left[\frac{m_{1}}{q_{1}}\right]-p^{i_{1}-j_{1}} \triangle_{1}\right)-\left(i_{1}-j_{1}+1\right) } \\
& \text { and since } \frac{m_{1}}{q_{1}}=\frac{1}{2} p^{i_{1}-j_{1}} \triangle_{1} \\
= & {\left[\frac{m}{q_{1}}\right]-\left(i_{1}-j_{1}+1\right) . }
\end{aligned}
$$

Thus, $v_{p}(T(s))+v_{p}\left(T\left(s^{0}\right)\right) \geqslant t_{j}^{s}-v_{p}\left(s_{i}\right)-s_{q}+v_{p}\left(a_{1}^{s_{1}} \cdots a_{q-1}^{s_{q-1}}\right)+\left[m / q_{1}\right]-$ $\left(i_{1}-j_{1}+1\right)$. Let $\varepsilon_{h}$ be defined as in the proof of Lemma 4.3.1. For $1 \leqslant h \leqslant 3$ and $t_{j}^{s} \geqslant\left[n / p^{s+j}(p-1)\right]-\varepsilon_{h} \geqslant[m / q]+\left[m_{1} / q\right]-\varepsilon_{h}$ and

$$
\begin{aligned}
v_{p}(T(s))+v_{p}\left(T\left(s^{0}\right)\right) \geqslant & {\left[\frac{m}{q}\right]-\varepsilon_{h}-v_{p}\left(s_{i}\right)-s_{q}+v_{p}\left(a_{1}^{s_{1}} \cdots a_{q-1}^{s_{q-1}}\right)+} \\
& +\left[\frac{m_{1}}{q}\right]+\left[\frac{m}{q_{1}}\right]-\left(i_{1}-j_{1}+1\right) \\
= & \theta_{h}(s)+\left[\frac{m_{1}}{q}\right]+\left[\frac{m}{q_{1}}\right]-\left(i_{1}-j_{1}+1\right) .
\end{aligned}
$$

For $h=4$, let $t_{j}^{s} \in T_{k}^{s}-\left\{t_{k}^{s}\right\}$ as in the proof of Lemma 4.3.1. We have $2 m+2 m_{1}$ in the place of $2 m$ in inequalities (4.1) and (4.2) holds as it is and we obtain from these two inequalities that $[m / q]+\left[m_{1} / q\right] \leqslant t_{j}^{s}$ and $[m / q]=s_{q}$. Hence

$$
\begin{aligned}
v_{p}(T(s))+v_{p}\left(T\left(s^{0}\right)\right) \geqslant & {\left[\frac{m}{q}\right]-v_{p}\left(s_{i}\right)-s_{q}+v_{p}\left(a_{1}^{s_{1}} \cdots a_{q-1}^{s_{q-1}}\right)+} \\
& +\left[\frac{m_{1}}{q}\right]+\left[\frac{m}{q_{1}}\right]-\left(i_{1}-j_{1}+1\right) \\
= & \theta_{h}(s)+\left[\frac{m_{1}}{q}\right]+\left[\frac{m}{q_{1}}\right]-\left(i_{1}-j_{1}+1\right) \text { as claimed. }
\end{aligned}
$$


To prove that $\left[m_{1} / q\right]+\left[m / q_{1}\right]-\left(i_{1}-j_{1}+1\right) \geqslant 0$ we consider the following two cases:

(i) $j \leqslant j_{1}$. Then $\left[m_{1} / q\right]=p^{i_{1}-j} \Delta_{1} \geqslant p^{i_{1}-j} \geqslant i_{1}-j+1 \geqslant i_{1}-j_{1}+1$.

(ii) $j>j_{1}$. Let $s_{q} \geqslant 1$. Then $\left[m / q_{1}\right] \geqslant\left[q s_{q} / q_{1}\right] \geqslant\left[q / q_{1}\right]=p^{j-j_{1}}$ and $\left[m_{1} / q\right]=$ $p^{i_{1}-j} \Delta \geqslant p^{i_{1}-j}$. Thus, $\left[m / q_{1}\right]+\left[m_{1} / q\right] \geqslant p^{i_{1}-j}+p^{j-j_{1}} \geqslant\left(i_{1}-j+1\right)+$ $\left(j-j_{1}+1\right)=i_{1}-j_{1}+2$. Since we are in case (ii) of the proof of Lemma 4.3.1, $s_{i}>0$ for $i \geqslant(p-1) p^{s+j-1}$ and hence $m \geqslant i s_{i} \geqslant(p-1) p^{s+j-1}$.

(a) Let $i_{1} \geqslant j$. Then $m / q_{1} \geqslant(p-1) p^{s+j-1} /(1 / 2)(p-1) p^{s+j_{1}}=2 p^{j-j_{1}-1} \geqslant$ $2\left(j-j_{1}\right),\left[m_{1} / q\right]=p^{i_{1}-j} \Delta_{1} \geqslant p^{i_{1}-j} \geqslant i_{1}-j+1$ and, thus, $\left[m / q_{1}\right]+$ $\left[m_{1} / q\right] \geqslant\left(i_{1}-j+1\right)+2\left(j-j_{1}\right)=\left(i_{1}-j_{1}+1\right)+\left(j-j_{1}\right)>i_{1}-j_{1}+1$.

(b) Let $i_{1}<j$. Then as in case $a$,

$$
\begin{aligned}
m / q_{1} \geqslant 2\left(j-j_{1}\right) & =\left(i_{1}-j_{1}+1\right)+\left(j-i_{1}\right)+\left(j-1-j_{1}\right) \\
& >i_{1}-j_{1}+1 .
\end{aligned}
$$

\section{LEMMA 4.3.5. Let}

$k \geqslant 2, \quad 0 \leqslant s \leqslant r_{n}, \quad 0 \leqslant j_{h}<i_{h}, i_{h} \geqslant 1, \quad m_{h}=(1 / 2)(p-1) p^{s+i_{h}} \triangle_{h}$, $2 m_{h} \leqslant n,\left(\triangle_{h}, p\right)=1(1 \leqslant h \leqslant k)$.

If $n \geqslant 2 m_{1}+2 m_{2}+\cdots+2 m_{k}$, then $\prod_{h=1}^{k} T\left(s^{0}\left(s, j_{h}, m_{h}\right)\right) \in \mathbb{Z}$.

Proof. It suffices to consider the cases $k=2,3$. For $k=2$, by Lemma 4.2.4,

$$
\begin{aligned}
& v_{p}\left(T\left(s^{0}\left(s, j_{1}, m_{1}\right)\right)\right)+v_{p}\left(T\left(s^{0}\left(s, j_{2}, m_{2}\right)\right)\right) \\
& \quad=t_{j_{1}}^{s}+t_{j_{2}}^{s}-p^{i_{1}-j_{1}} \triangle_{1}-\left(i_{1}-j_{1}\right)-p^{i_{2}-j_{2}} \Delta_{2}-\left(i_{2}-j_{2}\right) .
\end{aligned}
$$

By Lemma 4.1.12,

$$
t_{j_{1}}^{s}+t_{j_{2}}^{s} \geqslant\left[n /(p-1) p^{s+j_{1}}\right]+\left[n /(p-1) p^{s+j_{2}}\right]-2
$$

and since $n \geqslant 2 m_{1}+2 m_{2}, t_{j_{1}}^{s}+t_{j_{2}}^{s} \geqslant\left[m_{1} / q_{1}\right]+\left[m_{2} / q_{2}\right]+\left[m_{1} / q_{2}\right]+\left[m_{2} / q_{2}\right]-2$, where $q_{h}=(1 / 2)(p-1) p^{s+j_{h}} \triangle_{h},(h=1,2), m_{1} / q_{1}=p^{i_{1}-j_{1}} \triangle_{1}$ and $m_{2} / q_{2}=$ $p^{i_{2}-j_{2}} \triangle_{2}$ and, thus,

$$
\begin{aligned}
& v_{p}\left(T\left(s^{0}\left(s, j_{1}, m_{1}\right)\right)\right)+v_{p}\left(T\left(s^{0}\left(s, j_{2}, m_{2}\right)\right)\right) \\
& \quad \geqslant\left(\left[\frac{m_{1}}{q_{2}}\right]-\left(i_{1}-j_{2}+1\right)\right)+\left(\left[\frac{m_{2}}{q_{1}}\right]-\left(i_{2}-j_{1}+1\right)\right),
\end{aligned}
$$

$m_{1} / q_{2}=p^{i_{1}-j_{2}} \triangle_{1} \geqslant p^{i_{1}-j_{2}} \geqslant i_{1}-j_{2}+1$ and, hence, $\left[m_{1} / q_{2}\right] \geqslant i_{1}-j_{2}+1$ and, similarly, $\left[m_{2} / q_{1}\right] \geqslant i_{2}-j_{1}+1$.

For the case $k=3$, again by Lemma 4.2.4,

$$
\begin{aligned}
& \sum_{k=1}^{3} v_{p}\left(T\left(s^{0}\left(s, j_{h}, m_{h}\right)\right)\right) \\
& \quad=t_{j_{1}}^{s}+t_{j_{2}}^{s}+t_{j_{3}}^{s}-p^{i_{1}-j_{1}} \triangle_{1}-\left(i_{1}-j_{1}\right)-p^{i_{2}-j_{2}} \triangle_{2}- \\
& \quad-\left(i_{2}-j_{2}\right)-p^{i_{3}-j_{3}} \triangle_{3}-\left(i_{3}-j_{3}\right),
\end{aligned}
$$


and by exactly the same argument as for the case $k=2$,

$$
\begin{aligned}
\sum_{k=1}^{3} v_{p}\left(T\left(s^{0}\left(s, j_{h}, m_{h}\right)\right)\right) & \\
\geqslant & \left(\left[\frac{m_{1}}{q_{2}}\right]-\left(i_{1}-j_{2}+1\right)\right)+\left(\left[\frac{m_{2}}{q_{3}}\right]-\left(i_{2}-j_{3}+1\right)\right)+ \\
& +\left(\left[\frac{m_{3}}{q_{1}}\right]-\left(i_{3}-j_{1}+1\right)\right)+\left[\frac{m_{1}}{q_{3}}\right]+\left[\frac{m_{2}}{q_{1}}\right]+\left[\frac{m_{3}}{q_{2}}\right] \geqslant 0 .
\end{aligned}
$$

PROPOSITION 4.3.6. Let $k \geqslant 2,0 \leqslant s \leqslant r_{n}, 0 \leqslant j_{h} \leqslant r_{n}-s, 1 \leqslant m_{h} \leqslant[n / 2]$ and $s_{h} \in S_{m_{h}}^{s, j_{h}},(1 \leqslant h \leqslant k)$. If $n \geqslant 2 m_{1}+2 m_{2}+\cdots+2 m_{k}$ then $\prod_{h=1}^{k} T\left(s_{h}\right) \in \mathbb{Z}$.

Proof. If $s_{h}$ is not a distinguished sequence $\forall 1 \leqslant h \leqslant k$, the result follows from Lemma 4.3.1. Assume without loss of generality that $s_{h}$ is a distinguished sequence for $1 \leqslant h \leqslant l$ and $s_{h}$ is not a distinguished sequence for $l+1 \leqslant h \leqslant k$. If $l \geqslant 2$ then it follows from Lemma 4.3.5 that $\prod_{h=1}^{l} T\left(s_{h}\right) \in \mathbb{Z}$ and from Lemma 4.3.1 that $\prod_{h=l+1}^{k} T\left(s_{h}\right) \in \mathbb{Z}$. If $l=1$ then it follows from Lemma 4.3.4 that $T\left(s_{1}\right) T\left(s_{2}\right) \in \mathbb{Z}$ and from Lemma 4.3.5 that $\prod_{h=3}^{k} T\left(s_{h}\right) \in \mathbb{Z}$.

COROLLARY 4.3.7. Let $k \geqslant 2, \quad 0 \leqslant s \leqslant r_{n}, 0 \leqslant j_{h} \leqslant r_{n}-s, 1 \leqslant m_{h} \leqslant[n / 2](1 \leqslant$ $h \leqslant k)$. If $n \geqslant 2 m_{1}+2 m_{2}+\cdots+2 m_{k}$ then $\prod_{h=1}^{k} b_{m_{h}}^{s, j_{h}} \in \mathbb{Z}$.

Proof. It follows from Definition 4.2.1 that $\prod_{h=1}^{k} b_{m_{h}}^{s, j_{h}}=\sum_{s_{1} \in S_{m_{1}}^{s, j j_{1}}} \sum_{s_{2} \in S_{m_{2}}^{s, j_{2}}} \cdots$ $\sum_{s_{k} \in S_{m_{k}}^{s, j_{k}}}\left(\prod_{h=1}^{k} T\left(s_{h}\right)\right)$ and $\prod_{h=1}^{k} T\left(s_{h}\right) \in \mathbb{Z}$ by Proposition 4.3.6.

COROLLARY 4.3.8. Let $k \geqslant 2,0 \leqslant s \leqslant r_{n}, j_{h}, m_{h}, \alpha_{h} \in \mathbb{Z}^{+}(1 \leqslant h \leqslant k)$ and expand

$$
\rho_{\mathbb{R}}^{p}\left(\psi_{\mathbb{R}}^{p^{s+j_{h}}}(\omega)\right)^{\alpha_{h} p^{t_{j h}^{s}}}=1+\sum_{m=1}^{[n / 2]} c_{m}^{s, j_{h}} \omega^{m} .
$$

Then $\prod_{h=1}^{k} c_{m}^{s, j_{h}} \in \mathbb{Z}$.

$$
\begin{aligned}
& \text { Proof. } 1+\sum_{m=1}^{[n / 2]} c_{m}^{s, j_{h}} \omega^{m}=\left(1+\sum_{m=1}^{[n / 2]} b_{m}^{s, j_{h}} \omega^{m}\right)^{\alpha_{h}} \text { and thus } \\
& c_{m}^{s, j_{h}}=\sum_{\substack{\alpha_{0}^{h}+\cdots+\alpha_{m}^{h}=\alpha_{h} \\
\alpha_{1}^{h}+2 \alpha_{2}^{h}+\cdots+m \alpha_{m}^{h}=m}} \frac{\alpha_{h} !}{\alpha_{0}^{h} ! \alpha_{1}^{h} ! \cdots \alpha_{m}^{h} !}\left(b_{1}^{s, j_{h}}\right)^{\alpha_{1}^{h}} \cdots\left(b_{m}^{s, j_{h}}\right)^{\alpha_{m}^{h}}, \\
& \prod_{h=1}^{k} c_{m_{h}}^{s, j_{h}}=\prod_{h=1}^{k}\left(\sum_{\substack{\alpha_{0}^{h}+\cdots+\alpha_{m h}^{h}=\alpha_{h} \\
\alpha_{1}^{h}+2 \alpha_{2}^{h}+\cdots+m_{h} \alpha_{m_{h}}^{h}=m_{h}}} \frac{\alpha_{h} !}{\alpha_{0}^{h} ! \alpha_{1}^{h} ! \cdots \alpha_{m}^{h} !}\left(b_{1}^{s, j_{h}}\right)^{\alpha_{1}^{h} \cdots\left(b_{m_{h}}^{s, j_{h}}\right)^{\alpha_{m_{h}}^{h}}}\right)
\end{aligned}
$$




$$
\begin{aligned}
& =\sum_{\substack{\alpha_{0}^{1}+\cdots+\alpha_{m_{1}}^{1}=\alpha_{1} \\
\alpha_{1}^{1}+2 \alpha_{2}^{1}+\cdots+m_{1} \alpha_{m_{1}}^{1}=m_{1}}} \sum_{\substack{\alpha_{0}^{k}+\cdots+\alpha_{m_{k}}^{k}=\alpha_{1} \\
\alpha_{1}^{k}+2 \alpha_{2}^{k}+\cdots+m_{k} \alpha_{m_{k}}^{k}=m_{k}}} \times \\
& \times\left(\frac{\alpha_{1} !}{\alpha_{0}^{1} ! \cdots \alpha_{m_{1}}^{1} !}\right) \cdots\left(\frac{\alpha_{k} !}{\alpha_{0}^{k} ! \cdots \alpha_{m_{k}}^{k} !}\right) \times \\
& \times\left(b_{1}^{s, j_{1}}\right)^{\alpha_{1}^{1}} \cdots\left(b_{m_{1}}^{s, j_{1}}\right)^{\alpha_{m_{1}}^{1}} \cdots \cdots\left(b_{1}^{s, j_{k}}\right)^{\alpha_{1}^{k}} \cdots\left(b_{m_{k}}^{s, j_{k}}\right)^{\alpha_{m_{k}}^{k}} \text {. }
\end{aligned}
$$

Since $k \geqslant 2$ and $n \geqslant 2 \alpha_{1} m_{1}+\cdots+2 \alpha_{k} m_{k} \geqslant 2 \alpha_{1}^{1} m_{1}+\cdots+2 \alpha_{m_{1}}^{1} m_{1}+\cdots+$ $2 \alpha_{1}^{k} m_{k}+\cdots+2 \alpha_{m_{k}}^{k} m_{k}$. It follows from Corollary 4.3.7 that

$$
\left(b_{1}^{s, j_{1}}\right)^{\alpha_{1}^{1}} \cdots\left(b_{m_{1}}^{s, j_{1}}\right)^{\alpha_{m_{1}}^{1}} \cdots \cdots\left(b_{1}^{s, j_{k}}\right)^{\alpha_{1}^{k}} \cdots\left(b_{m_{k}}^{s, j_{k}}\right)^{\alpha_{m_{k}}^{k}} \in \mathbb{Z} .
$$

COROLLARY 4.3.9. Let $\alpha \in \mathbb{Z}^{+}, 0 \leqslant s \leqslant r_{n}, 0 \leqslant j \leqslant r_{n}-s$ and expand $\rho_{\mathbb{R}}^{p}\left(\psi_{\mathbb{R}}^{p^{s+j}}\right.$ $(\omega))^{\alpha p^{t_{j}}}=1+\sum_{m=1}^{[n / 2]} c_{m}^{s, j} \omega^{m}$. Then $c_{m}^{s, j} \equiv \alpha b_{m}^{s, j}(\bmod \mathbb{Z})$. where

Proof. $1+\sum_{m=1}^{[n / 2]} c_{m}^{s, j} \omega^{m}=\left(1+\sum_{m=1}^{[n / 2]} b_{m}^{s, j} \omega^{m}\right)^{\alpha}$. Thus $c_{m}^{s, j}=\alpha b_{m}^{s, j}+d_{m}^{s, j}$,

$$
\begin{aligned}
& d_{m}^{s, j}=\sum_{\substack{\alpha_{0}+\alpha_{1}+\cdots+\alpha_{m-1}=\alpha \\
\alpha_{1}+2 \alpha_{2}+\cdots+(m-1) \alpha_{m-1}=m}} \frac{\alpha !}{\alpha_{0} ! \alpha_{1} ! \cdots \alpha_{m-1} !}\left(b_{1}^{s, j}\right)^{\alpha_{1}} \cdots\left(b_{m-1}^{s, j}\right)^{\alpha_{m-1}}, \\
& n \geqslant 2 m=2\left(\alpha_{1}+2 \alpha_{2}+\cdots+(m-1) \alpha_{m-1}\right) \\
& =2 \alpha_{1}+4 \alpha_{2}+\cdots+2(m-1) \alpha_{m-1}
\end{aligned}
$$

and $\alpha_{1}+\alpha_{2}+\cdots+\alpha_{m-1} \geqslant 2$ and it follows from Corollary 4.3.7 that $\left(b_{1}^{s, j}\right)^{\alpha_{1}} \cdots\left(b_{m-1}^{s, j}\right)^{\alpha_{m-1}} \in \mathbb{Z}$ and thus $d_{m}^{s, j} \in \mathbb{Z}$.

COROLLARY 4.3.10. Let $\alpha_{j}^{s} \in \mathbb{Z} \quad\left(0 \leqslant s \leqslant r_{n}, 0 \leqslant j \leqslant r_{n}-s\right)$ and expand $\prod_{j=0}^{r_{n}-s} \rho_{\mathbb{R}}^{p}\left(\psi_{\mathbb{R}}^{p^{s+j}}(\omega)\right)^{\alpha_{j}^{s} p^{t_{j}^{s}}}=1+\sum_{m=1}^{[n / 2]} a_{m}^{s} \omega^{m}$. Then $a_{m}^{s} \equiv \sum_{j=0}^{r_{n}-s} \alpha_{j}^{s} b_{m}^{s, j}(\bmod \mathbb{Z})$.

Proof. Let $\rho_{\mathbb{R}}^{p}\left(\psi_{\mathbb{R}}^{p^{s+j}}(\omega)\right)^{\alpha_{j}^{s} p^{t_{j}^{s}}}=1+\sum_{m=1}^{[n / 2]} c_{m}^{s, j} \omega^{m}$. Then by definition,

$$
a_{m}^{s}=\sum_{m_{0}+m_{1}+\cdots+m_{r_{n}-s}=m} c_{m_{0}}^{s, 0} c_{m_{1}}^{s, 1} \cdots c_{m_{r_{n}-s}^{s, r_{n}-s}} .
$$

If $m_{i}>0$ for more than once in the above product then $c_{m_{0}}^{s, 0} \cdots c_{m_{s}}^{s, r_{n}-s} \in \mathbb{Z}$ by Corollary 4.3.8. Hence the only possibly non-integral terms in the above sum correspond to $m_{i}=m$ for some $0 \leqslant i \leqslant r_{n}-s$ and $m_{j}=0, j \neq i$; e.g. the terms, $c_{m}^{s, j} \equiv \alpha_{j}^{s} b_{m}^{s, j}(\bmod \mathbb{Z})$ by Corollary 4.3.9. Hence $a_{m}^{s} \equiv \sum_{j=0}^{r_{n}-s} \alpha_{j}^{s} b_{m}^{s, j}(\bmod \mathbb{Z})$. 
COROLLARY 4.3.11. Let $\alpha_{j}^{s} \in \mathbb{Z}, \quad\left(0 \leqslant s \leqslant r_{n}, 0 \leqslant j \leqslant r_{n}-s\right)$ and expand $\prod_{j=0}^{r_{n}-s} \rho_{\mathbb{R}}^{p}\left(\psi_{\mathbb{R}}^{p^{s+j}}(\omega)\right)^{\alpha_{j}^{s} p^{t_{j}^{s}}}=1+\sum_{m=1}^{[n / 2]} a_{m}^{s} \omega^{m}$. If $b_{m}^{s, j} \in \mathbb{Z} \forall 0 \leqslant j \leqslant r_{n}-s$ then $a_{m}^{s} \in \mathbb{Z}$.

\subsection{THE BIJECTION BETWEEN $\mathcal{M}_{n, s}$ AND $\Phi_{n, s}^{0}$}

DEFINITION 4.4.1. Let $0 \leqslant s \leqslant r_{n}$. Then $1 \leqslant m \leqslant[n / 2]$ is called a singular $s$ exponent if and only if there exists $0 \leqslant j \leqslant r_{n}-s$ such that $b_{m}^{s, j} \notin \mathbb{Z}$ (equivalently, $\left.\varepsilon_{m}^{s, j}>0\right)$.

It follows from Corollary 4.3.2 that if $m$ is a singular $s$-exponent then it can be written in the form, $m=(1 / 2)(p-1) p^{s+i} \Delta(i \geqslant j,(\Delta, p)=1)$. If $m$ is not a singular $s$-exponent then $b_{m}^{s, j} \in \mathbb{Z}, \forall 0 \leqslant j \leqslant r_{n}-s$. We let $\mathcal{M}_{n, s}$ denote the set of singular $s$-exponents. Then $\mathcal{M}_{n}=\mathcal{M}_{n, 0} \supseteq \mathcal{M}_{n, 1} \supseteq \cdots \supseteq \mathcal{M}_{n, s} \supseteq \mathcal{M}_{n, s+1} \supseteq \cdots$.

LEMMA 4.4.2. Let $0 \leqslant s \leqslant r_{n}, i \geqslant 1$ and $m=(1 / 2)(p-1) p^{s+i} \Delta \in \mathcal{M}_{n, s}$, $1 \leqslant m \leqslant[n / 2],(\triangle, p)=1$. Then $t_{i}^{s}=\Delta-1$.

\section{Proof.}

(i) Assume $t_{i}^{s} \leqslant \Delta-2$. Then by Corollary 4.1.8,

$$
\begin{aligned}
n \leqslant & p^{s+i}(p-1) t_{i}^{s}+p^{s+1}\left(p^{i}-1\right) \leqslant p^{s+i}(p-1)(\Delta-2)+ \\
& +p^{s+1}\left(p^{i}-1\right)=2 m-p^{s+1}\left(p^{i}-2 p^{i-1}+1\right) \\
& =2 m-p^{s+1}\left[p^{i-1}(p-1)-\left(p^{i-1}-1\right)\right]<2 m \text { contradiction. }
\end{aligned}
$$

(ii) Assume $t_{i}^{s} \geqslant \Delta$. Then for $0 \leqslant j<i$, by definition,

$$
\varepsilon_{m}^{s, j}=p^{i-j} \Delta+i-j-t_{j}^{s} \leqslant p^{i-j} t_{i}^{s}+i-j-t_{j}^{s}<0
$$

by Corollary 4.1.7. This contradicts the fact that $m \in \mathcal{M}_{n, s}$. Hence $t_{i}^{s}=\Delta-1$.

LEMMA 4.4.3. Let $0 \leqslant s \leqslant r_{n}, i \geqslant 1$ and $m=(1 / 2)(p-1) p^{s+i} \Delta \in \mathcal{M}_{n, s}$, $1 \leqslant m \leqslant[n / 2],(\Delta, p)=1$. Then there exists a unique $0 \leqslant k<i$ such that $t_{k}^{s}=$ $p^{v} \Delta \in \Phi_{n, s}^{0}(v \geqslant 1), i=k+v$ and $t_{j}^{s}=p^{i-j} \Delta-1,(k+1 \leqslant j \leqslant i)$ and

$$
\varepsilon_{m}^{s, j}= \begin{cases}\nu, & j=k, \\ i-j+1, & k+1 \leqslant j \leqslant i .\end{cases}
$$

Proof. Assume $t_{k}^{s} \notin \Phi_{n, s}^{0}, \forall 0 \leqslant k<i$. Then

$$
\begin{array}{rlrl}
n & \leqslant p^{s+i}(p-1) t_{i}^{s}+p^{s}(p-1)\left(p^{i}-1\right) & & \text { by Corollary 4.1.8 } \\
& =p^{s+i}(p-1)(\Delta-1)+p^{s}(p-1)\left(p^{i}-1\right) & & \text { by Proposition 4.4.4 } \\
& =2 m-p^{s}(p-1)<2 m \text { contradiction. } &
\end{array}
$$

Thus, there exists $0 \leqslant k<i$ such that $t_{k}^{s} \in \Phi_{n, s}^{0}$. Let $0 \leqslant k<i$ be the greatest integer such that $t_{k}^{s} \in \Phi_{n, s}^{0}$. By Observation 4.1.6, $t_{k+1}^{s} \leqslant p^{i-k-1}(\triangle-1)+p^{i-k-1}-1$ $=p^{i-k-1} \triangle-1$ and since $t_{k}^{s} \in \Phi_{n, s}^{0}, t_{k}^{s}=p\left(t_{k+1}^{s}+1\right) \leqslant p^{i-k} \Delta$. There are two cases 
to distinguish: Case 1. $t_{l}^{s}=p t_{l+1}^{s}+p-1, \forall k<l<i$. By Observation 4.1.6, $t_{k+1}^{s}=p^{i-k-1} \triangle-1$ and thus $t_{k}^{s}=p^{i-k} \triangle$. Case 2. $t_{l}^{s} \leqslant p t_{l+1}^{s}+p-2$ for some $k<l<i$. Then by Observation 4.1.6,

$$
\begin{aligned}
& t_{l+1}^{s} \leqslant p^{i-l-1} t_{i}^{s}+p^{i-l-1}-1 \leqslant p^{i-l-1}(\Delta-1)+p^{i-l-1}-1=p^{i-l-1} \Delta-1 \\
& t_{l}^{s} \leqslant p t_{l+1}^{s}+p-2 \leqslant p\left(p^{i-l-1} \Delta-1\right)+p-2=p^{i-l} \Delta-2 .
\end{aligned}
$$

By Observation 4.1.6,

$$
\begin{aligned}
t_{k+1}^{s} & \leqslant p^{l-k-1} t_{l}^{s}+p^{l-k-1}-1 \leqslant p^{l-k-1}\left(p^{i-l} \Delta-2\right)+p^{l-k-1}-1 \\
& =p^{i-k-1} \Delta-p^{l-k-1}-1
\end{aligned}
$$

and since $t_{k}^{s} \equiv 0(\bmod p), t_{k}^{s}=p t_{k+1}^{s}+p \leqslant p^{i-k} \Delta-p^{l-k} \leqslant p^{i-k} \Delta-p$ since $l>k$. By Corollary 4.1.8,

$$
\begin{aligned}
n \leqslant & p^{s+k}(p-1) t_{k}^{s}+p^{s+1}\left(p^{k}-1\right) \leqslant p^{s+k}(p-1)\left(p^{i-k} \Delta-p\right)+ \\
& +p^{s+1}\left(p^{k}-1\right)=p^{s+i}(p-1) \Delta-p^{s+1}\left(p^{k}(p-2)+1\right) \\
= & 2 m-p^{s+1}\left(p^{k}(p-2)+1\right)<2 m .
\end{aligned}
$$

Thus, the second case leads to a contradiction. By the first case, $t_{k}^{s}=p^{i-k} \triangle$. That $t_{j}^{s}=p^{i-j} \Delta-1,(k+1 \leqslant j \leqslant i)$ follows from Definition 4.1.9.

$$
\varepsilon_{m}^{s, k}=p^{i-k} \Delta+i-k-t_{k}^{s}=p^{v} \Delta+v-p^{v} \Delta=v
$$

and for $k+1 \leqslant j \leqslant i, \varepsilon_{m}^{s, j}=p^{i-j} \Delta+i-j-t_{j}^{s}=p^{i-j} \Delta+i-j-\left(p^{i-j} \Delta-1\right)=$ $i-j+1$.

PROPOSITION 4.4.4. There exists a bijection, $\sigma: \Phi_{n, s}^{0} \mapsto \mathcal{M}_{n, s}$ given by $\sigma\left(t_{k}^{s}\right)=$ $(1 / 2)(p-1) p^{s+k} t_{k}^{s}$.

Proof. Let $t_{k}^{s} \in \Phi_{n, s}^{0}$. Express $t_{k}^{s}=p^{v} \Delta, \quad(v \geqslant 1,(\Delta, p)=1)$ and define $\sigma\left(t_{k}^{s}\right)=(1 / 2)(p-1) p^{s+k+v} \Delta=(1 / 2)(p-1) p^{s+k} t_{k}^{s} \in \mathcal{M}_{n, s}$. By Lemma 4.4.3, $\sigma$ is invertible.

COROLLARY 4.4.5. $\Phi_{n}^{0}=\Phi_{n, 0}^{0} \supseteq \Phi_{n, 1}^{0} \supseteq \cdots \supseteq \Phi_{n, s}^{0} \supseteq \Phi_{n, s+1}^{0} \supseteq \cdots$.

Proof. It follows from Proposition 4.4.4 and Definition 4.4.1.

LEMMA 4.4.6. Let

$$
\begin{array}{ll}
0 \leqslant s \leqslant r_{n}, \quad 0 \leqslant k \leqslant r_{n}-s, & t_{k}^{s}=p^{\nu} \Delta \in \Phi_{n, s}^{0}, \\
(v \geqslant 1,(\triangle, p)=1), \quad i=k+v, & m=\sigma\left(t_{k}^{s}\right)=(1 / 2)(p-1) p^{s+i} \Delta .
\end{array}
$$


Then for $j \leqslant k$,

$$
p^{i-j} \Delta+\frac{p^{k-j}-1}{p-1} \leqslant t_{j}^{s} \leqslant p^{i-j} \Delta+\frac{p\left(p^{k-j}-1\right)}{p-1} .
$$

For $j<k$, either the left or the right inequality is strict.

Proof. By induction on $j$ starting with $j=k$ and $t_{k}^{s}=p^{v} \Delta$ and using the inequalities, $p t_{j+1}^{s}+1 \leqslant t_{j}^{s} \leqslant p\left(t_{j+1}^{s}+1\right)$.

COROLLARY 4.4.7. Let $0 \leqslant s \leqslant r_{n}, 0 \leqslant k \leqslant r_{n}-s, t_{k}^{s}=p^{\nu} \Delta \in \Phi_{n, s}^{0},(v \geqslant 1$, $(\Delta, p)=1), m=\sigma\left(t_{k}^{s}\right)=(1 / 2)(p-1) p^{s+k+v} \Delta$. Then $\left\{\varepsilon_{m}^{s, j}\right\}_{j \leqslant k-1}$ is a strictly increasing sequence bounded above by $\nu . \varepsilon_{m}^{s, k-1}=\nu$ if and only if $t_{k-1}^{s}=p^{\nu+1} \Delta+1$.

Proof. Let $j \leqslant k \cdot \varepsilon_{m}^{s, j}=p^{i-j} \Delta+i-j-t_{j}^{s}, \varepsilon_{m}^{s, j-1}=p^{i-j+1} \Delta+i-j+1-t_{j-1}^{s}-1$

(1) $\varepsilon_{m}^{s, j}-\varepsilon_{m}^{s, j-1}=\left(t_{j-1}^{s}-t_{j}^{s}\right)-\left((p-1) p^{i-j} \Delta+1\right)$. By Lemma 4.4.6,

(2) $t_{j-1}^{s} \geqslant p^{i-j+1} \Delta+\left(p^{k-j+1}-1\right) /(p-1)$; and

(3) $t_{j}^{s} \leqslant p^{i-j} \Delta+\left(p\left(p^{k-j}-1\right) /(p-1)\right.$; thus,

(4) $t_{j-1}^{s}-t_{j}^{s} \geqslant(p-1) p^{i-j} \Delta+1$.

Hence, $\varepsilon_{m}^{s, j}-\varepsilon_{m}^{s, j-1} \geqslant 0$. If we put $j=k$ in the above and note that $\varepsilon_{m}^{s, k}=v$ we obtain that $\varepsilon_{m}^{s, k-1} \leqslant v$. Let $j<k$. Then by Lemma 4.4.6, either inequality 2 or inequality 3 is strict and thus inequality 4 is strict, i.e. $\varepsilon_{m}^{s, j}-\varepsilon_{m}^{s, j-1}>0 . t_{k-1}^{s}=$ $p^{\nu+1} \Delta+\delta,(1 \leqslant \delta \leqslant p)$. Then

$$
\begin{aligned}
\varepsilon_{m}^{s, k-1} & =p^{k+\nu-(k-1)} \Delta+(k+v)-(k-1)-\left(p^{\nu+1} \Delta+\delta\right) \\
& =p^{\nu+1} \Delta+v+1-p^{\nu+1} \Delta-\delta \\
& =v+(1-\delta)=v \text { if and only if } \delta=1 .
\end{aligned}
$$

OBSERVATION 4.4.8. Let for $0 \leqslant s \leqslant r_{n}, 0 \leqslant h<k \leqslant r_{n}-s, t_{h}^{s}, t_{k}^{s} \in \Phi_{n, s}^{0}$ be consecutive elements such that $T_{h}^{s} \cap T_{k}^{s}=\left\{t_{k}^{s}\right\}$ and let $t_{k}^{s}=p^{v} \Delta(v \geqslant 1,(\Delta, p)=$ 1) and $m=(1 / 2)(p-1) p^{s+k+v} \Delta=\sigma\left(t_{k}^{s}\right) \in \mathcal{M}_{n, s}$. Then $\varepsilon_{m}^{k-1, s}=v$ if and only if $p=2$ and $t_{k-1}^{s}=2 t_{k}^{s}+1$.

Proof. Let $t_{h}^{s}=p^{\nu^{\prime}} \Delta^{\prime}\left(v^{\prime} \geqslant 1,\left(\Delta^{\prime}, p\right)=1\right)$. Then $t_{k-1}^{s}=p \Delta^{\prime}-1$ and $t_{k}^{s}=$ $\Delta^{\prime}-1=p^{v} \Delta$. Hence, $\Delta^{\prime}=p^{v} \Delta+1$ and $t_{k-1}^{s}=p\left(p^{v} \Delta+1\right)-1=p^{v+1} \Delta+p-1$. $\varepsilon_{m}^{s, k-1}=-t_{k-1}^{s}+k+v-(k-1)+p^{k+v-(k-1)} \Delta=-p^{v+1} \Delta-p+1+v+1+p^{v+1} \Delta=$ $v-(p-2)=v$ if and only if $p=2$. If $p=2, t_{k-1}^{s}=2^{v+1} \Delta+1$.

COROLLARY 4.4.9. Let $0 \leqslant s \leqslant r_{n}, 0 \leqslant k \leqslant r_{n}-s, t_{k}^{s}=p^{\nu} \Delta(v \geqslant 1,(\Delta, p)=$ 1), $p$ odd, $m=\sigma\left(t_{k}^{s}\right) \in \mathcal{M}_{n, s}$. Then, either, (i) $t_{k-1}^{s}>p^{v+1} \Delta+1$ in which case the sequence $\left\{\varepsilon_{m}^{s, j}\right\}_{j \leqslant k}$ is strictly increasing, or, (ii) $t_{k-1}^{s}=p^{v+1} \Delta+1$ in which case $\varepsilon_{m}^{s, k-1}=v$ and if $t_{h}^{s} \in \Phi_{n, s}^{0}$ is the preceding element to $t_{k}^{s}$ in $\Phi_{n, s}^{0}$ then $T_{h}^{s} \cap T_{k}^{s}=\emptyset$.

Proof. It is an immediate consequence of Corollary 4.4.7 and Observation 4.4.8. 


\section{5. $T\left(s^{0}\right)$}

LEMMA 4.5.1. Let $0 \leqslant s \leqslant r_{n}, 0 \leqslant j \leqslant i, i \geqslant 1$ and $m=(1 / 2)(p-1) p^{s+i} \Delta \in$ $\mathcal{M}_{n, s}, 1 \leqslant m \leqslant[n / 2],(\Delta, p)=1$. Then $T\left(s^{0}(s, j, m)\right) \equiv(-1)^{\mu_{m}^{s, j}}(1 / \Delta)\left(1 / p^{\varepsilon_{m i}^{s . j}}\right)$ $\left(\bmod \hat{Q}_{p}\right)$, where

$$
\mu_{m}^{s, j}= \begin{cases}p^{i-j} \Delta-1 & \text { if either } p \neq 2, \text { or, } m \text { is not the least element of } \mathcal{M}_{n, s}, \\ 1 & \text { or, } j \neq i-1\end{cases}
$$

Proof. By definition,

$$
T\left(s^{0}\right)=\left(\begin{array}{c}
c p^{t_{j}^{s}} \\
p^{i-j} \Delta
\end{array}\right)\left(\frac{1}{p}\right)^{p^{i-j} \Delta}
$$

i.e.

$$
T\left(s^{0}\right)=\frac{p^{t_{j}^{s}}}{p^{i-j} \Delta}\left(\frac{p^{t_{j}^{s}}}{p^{i-j} \Delta-1}-1\right)\left(\frac{p^{t_{j}^{s}}}{p^{i-j} \Delta-2}-1\right) \cdots\left(p^{t_{j}^{s}}-1\right) \frac{1}{p^{p^{i-j} \Delta}} .
$$

Let $\sigma\left(t_{k}^{s}\right)=m$, where $\sigma$ is the bijection of Proposition 4.4.4. We consider the following two cases. Case (i): $k+1 \leqslant j \leqslant i$ and Case (ii): $j \leqslant k$.

In Case (i), by Lemma 4.4.3, $t_{j}^{s}=p^{i-j} \Delta-1$. We divide into two sub-cases. (i)-a: $k+1 \leqslant j<i$, i.e. $i-j \geqslant 1$ and the terms $\left(p^{i-j} \Delta-l\right)\left(1 \leqslant l \leqslant p^{i-j} \Delta-1\right)$ appearing in the denominators of terms on the RHS are prime to $p$. It follows from Equation (4.3) that $T\left(s^{0}\right)=u+v$, where $v$ is the term obtained by multiplying all the (-1)'s together and $u$ is the sum of all products which contain at least a term $p^{t_{j}^{s}} /\left(p^{i-j} \Delta-l\right),\left(1 \leqslant l \leqslant p^{i-j} \Delta-1\right)$. Thus,

$$
v=(-1)^{p^{i-j} \Delta-1} \frac{1}{\Delta}\left(\frac{1}{p}\right)^{p^{i-j} \Delta+i-j-t_{j}^{s}}=(-1)^{p^{i-j} \Delta-1} \frac{1}{\Delta}\left(\frac{1}{p}\right)^{\varepsilon_{m}^{s, j}}
$$

On the other hand, $v_{p}(u) \geqslant 2 t_{j}^{s}-\left(p^{i-j} \Delta+i-j\right)=2\left(p^{i-j} \Delta-1\right)-\left(p^{i-j} \Delta+i-j\right)=$ $p^{i-j} \Delta-(i-j+2) \geqslant 0$ and, hence, $u \in \hat{Q}_{p}$ except for $p=2, \Delta=1, i-j=1$. For this exceptional case, $t_{j}^{s}=1, \varepsilon_{m}^{s, j}=2$.

$$
T\left(s^{0}\right)=\left(\begin{array}{l}
2 \\
2
\end{array}\right)\left(\frac{1}{2}\right)^{2^{1}}=\left(\frac{1}{2}\right)^{2}=\left(\frac{1}{2}\right)^{\varepsilon_{m}^{s, j}}
$$

Since $\Delta=1$ and $\mu_{m}^{s, j}=1$, this agrees with the statement of the lemma. (i)-b: $j=i$. Then $t_{j}^{s}=\Delta-1$ and it follows from Equation (4.3) that $T\left(s^{0}\right)=u+v$, where $v=(-1)^{p^{i-j} \Delta-1} 1 / \Delta(1 / p)^{\varepsilon_{m}^{s, j}}$ and

$$
u=\frac{1}{\Delta} \sum_{r=1}^{p^{i-j}} \sum_{1 \leqslant l_{1}<\cdots<l_{r}<\Delta} \frac{p^{r \Delta-(r+1)}}{\left(\Delta-l_{1}\right)\left(\Delta-l_{2}\right) \cdots\left(\Delta-l_{r}\right)} .
$$


$l_{h} \leqslant \Delta-1$ and thus $\Delta-l_{h} \leqslant \Delta-1 \leqslant p^{\Delta-2}$. Hence $v_{p}\left(\Delta-l_{h}\right) \leqslant \Delta-2$,

$$
v_{p}\left[\frac{p^{r \Delta-(r+1)}}{\left(\Delta-l_{1}\right) \cdots\left(\Delta-l_{r}\right)}\right] \geqslant r \Delta-(r+1)-r(\Delta-2)=r-1 \geqslant 0 .
$$

Hence $v_{p}(u) \geqslant 0$, i.e. $u \in \hat{Q}_{p}$.

Case (ii): $j \leqslant k$. The proof is as in Case (i-a). $T\left(s^{0}\right)=u+v$, where $v=$ $(-1)^{p^{i-j} \Delta-1}(1 / \Delta)(1 / p)^{\varepsilon_{m}^{s . j}}$ and

$$
\begin{aligned}
v_{p}(u) & \geqslant 2 t_{j}^{s}-\left(p^{i-j} \Delta+i-j\right) \\
& \geqslant 2\left(p^{i-j} \Delta+\frac{\left(p^{k-j}-1\right)}{(p-1)}\right)-\left(p^{i-j} \Delta+i-j\right), \text { by Lemma } 4.4 .6 \\
& =\left[p^{i-j} \Delta-(i-j)\right]+\frac{\left(p^{k-j}-1\right)}{(p-1)}>0 \text {, i.e. } u \in \hat{Q}_{p} .
\end{aligned}
$$

COROLLARY 4.5.2. Let $0 \leqslant s \leqslant r_{n}, 0 \leqslant j \leqslant i, i \geqslant 1$ and $m=(1 / 2)(p-1) p^{s+i} \Delta \in$ $\mathbb{\Downarrow}, 1 \leqslant m \leqslant[n / 2],(\Delta, p)=1$. Then $b_{m}^{s, j} \equiv(-1)^{\tau_{m}^{s, j}}(1 / \Delta)\left(1 / p^{\varepsilon_{m}^{s, j}}\right)\left(\bmod \hat{Q}_{p}\right)$, where

$$
\tau_{m}^{s, j}= \begin{cases}\triangle-1 & \text { if } p \text { is odd } \\
1 & \begin{array}{l}
\text { if } p=2, j<i \text { and if either } m \text { is not the least } \\
\text { element of } \mathcal{M}_{n, s} \text { or } j \neq i-1,
\end{array} \\
0 & \text { if } p=2, m \text { is the least element of } \mathcal{M}_{n, s} \text { and } j=i-1, \\
0 & \text { if } p=2 \text { and } j=i .\end{cases}
$$

Proof. $b_{m}^{s, j}=T\left(s^{0}\right)+\sum_{s \neq s^{0}} T(s) \equiv T\left(s^{0}\right)(\bmod \mathbb{Z})$ by Lemma 4.3.1 The result follows from Lemma 4.5.1 by noting that $\mu_{m}^{s, j} \equiv \tau_{m}^{s, j}(\bmod 2)$.

COROLLARY 4.5.3. Let $0 \leqslant s \leqslant r_{n}, \alpha_{j}^{s} \in \mathbb{Z}\left(0 \leqslant j \leqslant r_{n}-s\right)$ and

$$
\prod_{j=0}^{r_{n}-s} \rho_{\mathbb{R}}^{p}\left(\psi_{\mathbb{R}}^{p^{s+j}}(\omega)\right)^{\alpha_{j}^{s} p^{s_{j}^{s}}}=1+\sum_{m=1}^{[n / 2]} a_{m}^{s} \omega^{m}
$$

If $m=(1 / 2)(p-1) p^{i} \Delta \in \mathcal{M}_{n, s}(i \geqslant 1,(\Delta, p)=1)$ then

$$
a_{m}^{s} \equiv \begin{cases}\mp \frac{1}{\Delta} \sum_{j=0}^{i} \frac{\alpha_{j}^{s}}{p^{s, j}}\left(\bmod \hat{Q}_{p}\right) & \text { if either } p \neq 2 \text { or } m \text { is } n o t \\ & \text { the least element of } \mathcal{M}_{n, s}, \\ -\frac{1}{\Delta} \sum_{\substack{0 \leqslant j \leqslant i \\ j \neq i-1}} \frac{\alpha_{j}^{s}}{2^{\varepsilon_{m}^{s, j}}}+\frac{1}{\Delta} \frac{\alpha_{i-1}^{s}}{2^{\varepsilon_{m}^{s, i}}}\left(\bmod \hat{Q}_{2}\right) & \text { if } p=2 \text { and } m \text { is the least } \\ \text { element of } \mathcal{M}_{n, s} .\end{cases}
$$

Proof. By Corollary 4.3.10,

$$
a_{m}^{s} \equiv \sum_{j=0}^{r_{n}-s} \alpha_{j}^{s} b_{m}^{s, j}=\sum_{j=0}^{i} \alpha_{j}^{s} b_{m}^{s, j}+\sum_{j=i+1}^{r_{n}-s} \alpha_{j}^{s} b_{m}^{s, j}(\bmod \mathbb{Z}) .
$$


By Corollary 4.3.2, $b_{m}^{s, j} \in \mathbb{Z}$ for $j>i$. Thus, $a_{m}^{s} \equiv \sum_{j=0}^{i} \alpha_{j}^{s} b_{m}^{s, j}(\bmod \mathbb{Z})$.

If $p$ is odd, $\tau_{m}^{s, j}=\Delta-1$ and by Corollary 4.5.2,

$$
a_{m}^{s} \equiv \frac{(-1)^{\Delta-1}}{\triangle} \sum_{j=0}^{i} \frac{\alpha_{j}^{s}}{p^{\varepsilon_{m}^{s, j}}}\left(\bmod \hat{Q}_{p}\right) \equiv \mp \frac{1}{\triangle} \sum_{j=0}^{i} \frac{\alpha_{j}^{s}}{p^{\varepsilon_{m}^{s, j}}}\left(\bmod \hat{Q}_{p}\right) .
$$

If $p=2$ and $m$ is not the least element of $\mathcal{M}_{n, s}$,

$$
\tau_{m}^{s, j}= \begin{cases}1, & j<i \\ 0, & j=i\end{cases}
$$

Hence

$$
a_{m}^{s} \equiv-\frac{1}{\triangle} \sum_{j=0}^{i-1} \frac{\alpha_{j}^{s}}{2^{\varepsilon_{m}^{s, j}}}+\frac{1}{\triangle} \frac{\alpha_{i}^{s}}{2^{\varepsilon_{m}^{s, i}}} \quad\left(\bmod \hat{Q}_{2}\right)
$$

By Lemma 4.4.3, $\varepsilon_{m}^{s, i}=1$ and thus,

$$
(1 / \Delta)\left(\alpha_{i}^{s} / 2^{\varepsilon_{m}^{s, i}}\right) \equiv-(1 / \triangle)\left(\alpha_{i}^{s} / 2^{\varepsilon_{m}^{s, i}}\right) \quad\left(\bmod \hat{Q}_{2}\right) .
$$

Hence

$$
a_{m}^{s} \equiv-\frac{1}{\triangle} \sum_{j=0}^{i-1} \frac{\alpha_{j}^{s}}{2^{s, j}}-\frac{1}{\triangle} \frac{\alpha_{i}^{s}}{2^{\varepsilon_{m}^{s, i}}}\left(\bmod \hat{Q}_{2}\right) \equiv \frac{1}{\triangle} \sum_{j=0}^{i} \frac{\alpha_{j}^{s}}{2^{\varepsilon_{m}^{s, j}}}\left(\bmod \hat{Q}_{2}\right)
$$

For $p=2$ and $m$ is the least element of $\mathcal{M}_{n, s}$,

$$
\tau_{m}^{s, j}= \begin{cases}1, & j<i-1, \\ 0, & j=i-1, i\end{cases}
$$

and

$$
\begin{aligned}
a_{m}^{s} & \equiv-\frac{1}{\triangle} \sum_{j=0}^{i-2} \frac{\alpha_{j}^{s}}{2^{\varepsilon_{m}^{s, j}}}+\frac{1}{\triangle} \frac{\alpha_{i-1}^{s}}{2^{\varepsilon_{m}^{s, i-1}}}+\frac{\alpha_{i}^{s}}{2^{\varepsilon_{m}^{s, i}}} \quad\left(\bmod \hat{Q}_{2}\right) \\
& \equiv-\frac{1}{\triangle} \sum_{j=0}^{i-2} \frac{\alpha_{j}^{s}}{2^{\varepsilon_{m}^{s, j}}}+\frac{1}{\Delta} \frac{\alpha_{i-1}^{s}}{2^{\varepsilon_{m}^{s, i-1}}}-\frac{1}{\triangle} \frac{\alpha_{i}^{s}}{2^{\varepsilon_{m}^{s, i}}} \quad\left(\bmod \hat{Q}_{2}\right) \\
& \equiv-\frac{1}{\triangle} \sum_{\substack{0 \leq j \leq i \\
j \neq i-1}}^{i-1} \frac{\alpha_{j}^{s}}{2^{\varepsilon_{m}^{s, j}}}+\frac{1}{\triangle} \frac{\alpha_{i-1}^{s}}{2^{\varepsilon_{m}^{s, i-1}}}\left(\bmod \hat{Q}_{2}\right) .
\end{aligned}
$$




\section{Admissible Sequences}

\subsection{EXTENSION OF ADMISSIBLE SEQUENCES}

DEFINITION 5.1.1. Let $0 \leqslant s \leqslant r_{n}, \mathcal{M}_{n, s} \neq \emptyset, m=\inf \left(\mathcal{M}_{n, s},(\Delta, p)=1\right), m=$ $(1 / 2)(p-1) p^{s+i} \Delta, 1 \leqslant m \leqslant[n / 2],(\Delta, p)=1$. Define $r_{n, s}=i$. Then $r_{n, s} \leqslant r_{n}-s$ and for $p=2$, it follows as in the proof of Corollary 4.1.13 that if $\Phi_{n, s}^{0} \neq \emptyset$, then $r_{n, s}=r_{n}-s$.

DEFINITION 5.1.2. Let $0 \leqslant s \leqslant r_{n}, m \in \mathcal{M}_{n, s}$. Define $\mathcal{M}_{n, s}^{m}=\left\{m^{\prime} \in \mathcal{M}_{n, s}\right.$ : $\left.m^{\prime} \geqslant m\right\}$.

DEFINITION 5.1.3. Let $0 \leqslant s \leqslant r_{n}, m=(1 / 2)(p-1) p^{s+i} \Delta \in \mathcal{M}_{n, s}, i \geqslant 1,(\Delta$, $p)=1$. A sequence of integers $\alpha=\left(\alpha_{j}\right)_{0 \leqslant j \leqslant i}$ is called admissible in $\mathcal{M}_{n, s}^{m}$ if and only if $\sum_{j=0}^{i^{\prime}}\left(\alpha_{j} / p^{\varepsilon_{m^{\prime}}^{s, j}}\right) \in \mathbb{Z}, \forall m^{\prime}=(1 / 2)(p-1) p^{s+i^{\prime}} \Delta^{\prime} \in \mathcal{M}_{n, s}^{m}, 1 \leqslant i^{\prime} \leqslant i$, $\left(\Delta^{\prime}, p\right)=1$ unless $p=2$ and $m$ is the least element of $\mathcal{M}_{n, s}$, in which case, $\sum_{j=0}^{i^{\prime}}\left(\alpha_{j} / 2^{\varepsilon_{m^{\prime}}^{s, j}}\right) \in \mathbb{Z} \forall m^{\prime}>m$ and $\sum_{\substack{0 \leqslant j \leqslant i \\ j \neq i=1}}^{\substack{j \\ j \neq i}}\left(\alpha_{j} / 2^{\varepsilon_{m}^{s, j}}\right)-\left(\alpha_{i-1} / 2^{\varepsilon_{m}^{s, i-1}}\right) \in \mathbb{Z}$.

DEFINITION 5.1.4. Let $0 \leqslant s \leqslant r_{n}$ and $\mathcal{M}_{n, s} \neq \emptyset$. A sequence $\alpha=\left(\alpha_{j}\right)_{0 \leqslant j \leqslant r_{n, s}}$ is called $s$-admissible if and only if it is admissible in $\mathcal{M}_{n, s}^{m}, \forall m \in \mathcal{M}_{n, s}$. A zero admissible sequence is, simply, called admissible.

OBSERVATION 5.1.5. Let $0 \leqslant s \leqslant r_{n}$ and $m^{\prime}>m$ be consecutive elements of $\mathcal{M}_{n, s}, m^{\prime}=(1 / 2)(p-1) p^{s+i^{\prime}} \Delta^{\prime}, m=(1 / 2)(p-1) p^{s+i} \Delta$, where $i>i^{\prime} \geqslant 1,\left(\Delta^{\prime}\right.$, $p)=1$. Then a sequence $\alpha=\left(\alpha_{j}\right)_{0 \leqslant j \leqslant i}$ is admissible in $\mathcal{M}_{n, s}^{m}$ if and only if the subsequence $\left\{\alpha_{j}\right\}_{0 \leqslant j \leqslant i^{\prime}}$ is admissible in $\mathcal{M}_{n, s}^{m^{\prime}}$ and that $\sum_{j=0}^{i}\left(\alpha_{j} / p^{\varepsilon_{m}^{s, j}}\right) \in \mathbb{Z}$ if either $p \neq 2$ or $m$ is not the least element of $\mathcal{M}_{n, s}$ and

$$
\sum_{\substack{0 \leqslant j \leqslant i \\ j \neq i-1}}\left(\alpha_{j} / 2^{\varepsilon_{m}^{s, j}}\right)-\left(\alpha_{i-1} / 2^{\varepsilon_{m}^{s, i-1}}\right) \in \mathbb{Z}
$$

if $p=2$ and $m$ is the least element of $\mathcal{M}_{n, s}$.

LEMMA 5.1.6. Let $0 \leqslant s \leqslant r_{n}$ and $m^{\prime}>m$ be consecutive elements of $\mathcal{M}_{n, s}, m^{\prime}=$ $(1 / 2)(p-1) p^{s+i^{\prime}} \Delta^{\prime}, m=(1 / 2)(p-1) p^{s+i} \Delta, i<i^{\prime} \geqslant 1,\left(\Delta^{\prime}, p\right)=(\Delta, p)=1$. Let the sequence $\left\{\alpha_{j}\right\}_{0 \leqslant j \leqslant i^{\prime}}$ be admissible in $\mathcal{M}_{n, s}^{m^{\prime}}$. Then there exist integers $\alpha_{j}$ $\left(i^{\prime}<j \leqslant i\right)$ such that the sequence $\left\{\alpha_{j}\right\}_{0 \leqslant j \leqslant i}$ is admissible in $\mathcal{M}_{n, s}^{m}$.

Proof. Let $m^{\prime}=\sigma\left(t_{k^{\prime}}\right)=\sigma\left(p^{\nu^{\prime}} \Delta^{\prime}\right)$ and $m=\sigma\left(t_{k}\right)=\sigma\left(p^{\nu} \Delta\right),\left(\Delta^{\prime}, p\right)=$ $(\Delta, p)=1, i^{\prime}=k^{\prime}+v^{\prime}, i=k+v$. If $i^{\prime}<k$ put $\alpha_{l}=0\left(i^{\prime}<l \leqslant k\right)$ and hence assume that $\left\{\alpha_{l}\right\}$ is defined for $0 \leqslant l \leqslant k$. It follows from Corollary 4.4.7 and Lemma 4.4.3 that $\left(\varepsilon_{m}^{s, j}\right)_{k<j \leqslant i}=(v v-1 \cdots 21)$ and $\left(\varepsilon_{m}^{s, j}\right)_{j \leqslant k-1}$ is a strictly increasing sequence bounded above by $\nu$ and $\varepsilon_{m}^{s, k}=v$. Hence for $k<l \leqslant i$, there 
exist at most two integers $g \leqslant k$ such that $\varepsilon_{m}^{s, g}=\varepsilon_{m}^{s, l}$. If either $p \neq 2$, or, $m$ is not the least element of $\mathcal{M}_{n, s}$ or $l \neq i-1$, put

$$
\alpha_{l}=-\sum_{\substack{g \leqslant k \\ s, g \\ \varepsilon_{m}^{s, g}=\varepsilon_{m}^{s, l}}} \alpha_{g},
$$

and if $p=2, m$ is the least element of $\mathcal{M}_{n, s}$ and $l=i-1$, put

$$
\alpha_{l}=\sum_{\substack{g \leqslant k \\ g \leqslant k \\ \varepsilon_{m}^{s, g}=\varepsilon_{m}^{s, i-1}}} \alpha_{g}
$$

This defines $\alpha_{l}(k<l \leqslant i)$ and it follows from Observation 5.1.5 that $\left\{\alpha_{j}\right\}_{0 \leqslant j \leqslant i}$ is admissible in $\mathcal{M}_{n, s}^{m}$.

PROPOSITION 5.1 .7 (Extension of admissible sequences). Let $0 \leqslant s \leqslant r_{n}, m \in$ $\mathcal{M}_{n, s}, m=(1 / 2)(p-1) p^{s+i} \Delta, i \geqslant 1,(\Delta, p)=1$ and let the sequence $\left\{\alpha_{j}\right\}_{0 \leqslant j \leqslant i}$ be admissible in $\mathcal{M}_{n, s}^{m}$. Then there exist integers $\alpha_{j}\left(i<j \leqslant r_{n, s}\right)$ such that the sequence $\left\{\alpha_{j}\right\}_{0 \leqslant j \leqslant r_{n, s}}$ is $s$-admissible.

Proof. It follows by induction from Lemma 5.1.6.

\section{2. $\alpha$ - AND $\beta$-SEQUENCES}

In this section, for $0 \leqslant s \leqslant r_{n}$, we shall construct the $\alpha$ - and $\beta$-sequences and show that they, in turn, give rise to $s$-admissible sequences.

PROPOSITION 5.2.1. Let $0 \leqslant s \leqslant r_{n}$. Then there exists a sequence $\left\{j_{h}\right\}_{0} \leqslant h \leqslant v$ $\left(v \leqslant r_{n}-s\right)$ with the following properties:

(i) $\left\{j_{h}\right\}_{0 \leqslant h \leqslant v}$ is strictly increasing.

(ii) $j_{0}=0$.

(iii) If $k_{0}$ is the smallest integer such that $t_{k_{0}}^{s} \in \Phi_{n, s}^{0}$ then $\left\{j_{h}\right\}_{0 \leqslant h \leqslant v} \cap\left[0, k_{0}\right]=$ $j_{0}=0$.

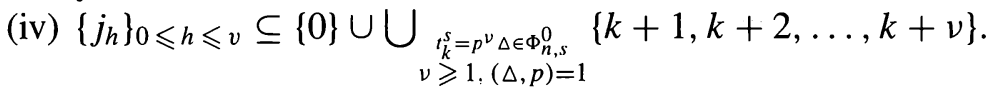

In particular, if $\Phi_{n, s}^{0}=\phi$ then $v=0$; i.e. the sequence consists of a single element $j_{0}=0$.

Proof. We put $j_{0}=0$ and if $\Phi_{n, s}^{0}=\phi$ then the sequence consists of this element only. If $\Phi_{n, s}^{0} \neq \phi$ then the sequence is constructed by induction on $t_{k}^{s} \in \Phi_{n, s}^{0}$ as follows. If $k_{0}$ is the smallest integer such that $t_{k_{0}}^{s} \in \Phi_{n, s}^{0}$ then we let $\left\{j_{h}\right\} \cap\left[0, k_{0}\right]=$ $j_{0}=0$. Let $t_{k}^{s}=p^{v} \Delta \in \Phi_{n, s}^{0}(v \geqslant 1,(\Delta, p)=1)$ and $m=\sigma\left(t_{k}^{s}\right)=(1 / 2)(p-1)$ 
$p^{s+k} t_{k}^{s} \in \mathcal{M}_{n, s}$. Assume that $\left\{j_{h}\right\} \cap[0, k]=\left\{j_{h}\right\}_{0 \leqslant h \leqslant \beta}$ has been constructed so that

$$
\left\{j_{h}\right\}_{0 \leqslant h \leqslant \beta} \subseteq\{0\} \cup \bigcup_{\substack{l<k, t_{l}^{s}=p^{\nu_{l}} \Delta_{l} \in \Phi_{n, s}^{0} \\ \nu_{l} \geqslant 1,\left(\Delta_{l}, p\right)=1}}\left\{l+1, l+2, \ldots, l+v_{l}\right\}
$$

and that properties (i)-(iii) are satisfied. We have

(1) $\varepsilon_{m}^{s, 0}<\varepsilon_{m}^{s, 1}<\cdots<\varepsilon_{m}^{s, k-1} \leqslant v$ by Corollary 4.4.7. Let $0 \leqslant \alpha \leqslant \beta$ be the smallest integer such that $\varepsilon_{m}^{s, j_{\alpha}}>0$. Then

(2) $j_{\alpha}<j_{\alpha+1}<\cdots<j_{\beta-1}<j_{\beta} \leqslant k$. It follows from 1 and 2 above that

(3) $0<\varepsilon_{m}^{s, j_{\alpha}}<\cdots<\varepsilon_{m}^{s, j_{\beta-1}}<\varepsilon_{m}^{s, j_{\beta}} \leqslant v$. Unless $j_{\beta-1}=k-1, j_{\beta}=k$ and $\varepsilon_{m}^{s, j_{\beta-1}}=\varepsilon_{m}^{s, j_{k-1}}=v$, in which case, $k>k_{0}$ since $\left\{j_{h}\right\} \cap\left[0, k_{0}\right]$ contains a single element, namely, $j_{0}=0$. Let $t_{l}^{s}=p^{\nu^{\prime}} \Delta^{\prime} \in \Phi_{n, s}^{0}\left(\nu^{\prime} \geqslant 1,\left(\Delta^{\prime}, p\right)=1\right)$ be the preceding element to $t_{k}^{s}$ in $\Phi_{n, s}^{0}$. Then $j_{\beta} \leqslant l+v^{\prime}$, i.e. $t_{k}^{s} \in T_{l}^{s} \cap T_{k}^{s}$. We have $\varepsilon_{m}^{s, k-1}=v$ and $T_{l}^{s} \cap T_{k}^{s} \neq \emptyset$ and it follows from Observation 4.4.8 that $p=2$ and $t_{k-1}^{s}=2 t_{k}^{s}+1$ in this exceptional case.

Thus, except in the exceptional case, it follows from (3) and the fact that $\left(\varepsilon_{m}^{s, j}\right)_{k+1 \leqslant j \leqslant k+v}=(\nu v-1 \cdots 21)$ that there exist unique elements, $k+1 \leqslant j_{\beta+1}<$ $\left.j_{\beta+2}<\cdots<j_{2 \beta-\alpha+1}\right] \leqslant k+v$ such that $\varepsilon_{m}^{s, j_{\beta+h}}=\varepsilon_{m}^{s, j_{\beta-h+1}}(1 \leqslant h \leqslant \beta-\alpha+1)$, or, equivalently that $j_{\beta+h}=k+v+1-\varepsilon_{m}^{s, j_{\beta-h+1}}$ (since $\varepsilon_{m}^{s, j_{\beta-h+1}}=\varepsilon_{m}^{s, j_{\beta+h}}=$ $\left.k+v+1-j_{\beta+h}\right)$. In the exceptional case, $p=2, j_{\beta-1}=k-1, j_{\beta}=k$, $\varepsilon_{m}^{s, k-1}=v, t_{k-1}^{s}=2 t_{k}^{s}+1$, we have $0 \leqslant j_{\alpha}<j_{\alpha+1}<\cdots<j_{\beta-2} \leqslant k-2$ and we deduce from 1 that 4. $\varepsilon_{m}^{s, j_{\alpha}}<\varepsilon_{m}^{s, j_{\alpha+1}}<\cdots<\varepsilon_{m}^{s, j_{\beta-2}} \leqslant v-1$. It follows from 4 and that $\left(\varepsilon_{m}^{s, j}\right)_{k+2 \leqslant j \leqslant k+\nu}=(v-1 v-2 \cdots 21)$ that there exist unique elements $k+2 \leqslant j_{\beta+1}<j_{\beta+2}<\cdots<j_{2 \beta-\alpha-1} \leqslant k+v$ such that $\varepsilon_{m}^{s, j_{\beta+h}}=$ $\varepsilon_{m}^{s, j_{\beta-h-1}}(1 \leqslant h \leqslant \beta-\alpha-1)$, or, equivalently that $j_{\beta+h}=k+v+1-\varepsilon_{m}^{s, j_{\beta-h-1}}$ (since $\varepsilon_{m}^{s, j_{\beta-h-1}}=\varepsilon_{m}^{s, j_{\beta+h}}=k+v+1-\varepsilon_{m}^{s, j_{\beta+h}}$ ).

Let $t_{k_{1}}^{s} \in \Phi_{n, s}^{0}$ be the consecutive element to $t_{k}^{s}$ in $\Phi_{n, s}^{0}$. This completes the construction of $\left\{j_{h}\right\}_{0 \leqslant h \leqslant 2 \beta-\alpha \mp 1}=\left\{j_{h}\right\} \cap\left[0, k_{1}\right]$ with the desired properties.

PROPOSITION 5.2.2. Let $0 \leqslant s \leqslant r_{n}$. The $\alpha$-sequence $\left\{\alpha_{j}\right\}_{0 \leqslant j \leqslant r_{n, s}}$, defined by

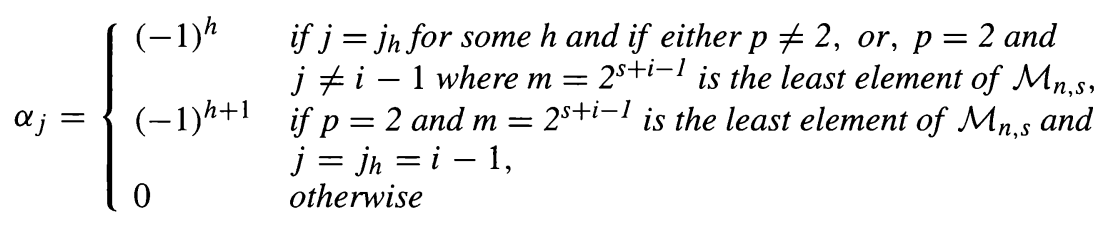

is an s-admissible sequence.

Proof. Let $0 \leqslant s \leqslant r_{n}$. We use the notation of the proof of Proposition 5.2.1. Let $t_{k}^{s}=p^{\nu} \Delta<\Phi_{n, s}^{0}(v \geqslant 1,(\Delta, p)=1)$ and $m=\sigma\left(t_{k}^{s}\right)=(1 / 2)(p-1) p^{s+k} t_{k}^{s} \in$ 
$\mathcal{M}_{n, s}$. Assume either $p \neq 2$ or $m$ is not the least element of $\mathcal{M}_{n, s}$. Except in the exceptional case, $p=2, j_{\beta-1}=k-1, j_{\beta}=k, \varepsilon_{m}^{s, k-1}=v, t_{k-1}^{s}=2 t_{k}^{s}+1$, we have

$$
\begin{aligned}
\sum_{j=0}^{k+v} \frac{\alpha_{j}}{2^{\varepsilon_{m}^{s, j}}} & =\sum_{0 \leqslant j_{h} \leqslant k+v} \frac{(-1)^{h}}{2^{\varepsilon_{m}^{s, j_{h}}}} \\
& =\sum_{h=0}^{\alpha-1} \frac{(-1)^{h}}{2^{\varepsilon_{m}^{s, j_{h}}}}+\sum_{h=\alpha}^{\beta-2} \frac{(-1)^{h}}{2^{\varepsilon_{m}^{s, j h}}}+\frac{(-1)^{\beta-1}}{2^{v}}+\frac{(-1)^{\beta}}{2^{v}}+\sum_{h=\beta+1}^{2 \beta-\alpha-1} \frac{(-1)^{h}}{2^{\varepsilon_{m}, j_{h}}} \\
& =\sum_{h=0}^{\alpha-1} \frac{(-1)^{h}}{2^{\varepsilon_{m}^{s, j_{h}}}}+\sum_{h=1}^{\beta-\alpha-1} \frac{(-1)^{\beta-h-1}}{2^{s, j_{\beta}-h-1}}+\sum_{h=1}^{\beta-\alpha-1} \frac{(-1)^{\beta+h}}{2^{\varepsilon_{m}, j_{\beta+h}}} \\
& =\sum_{h=0}^{\alpha-1} \frac{(-1)^{h}}{2^{\varepsilon_{m}^{s, j_{h}}}}+\sum_{h=1}^{\beta-\alpha-1} \frac{\left[(-1)^{\beta-h-1}+(-1)^{\beta+h}\right]}{2^{\varepsilon_{m}^{s, j_{\beta+h}}}}
\end{aligned}
$$

since $\varepsilon_{m}^{s, j_{\beta+h}}=\varepsilon_{m}^{s, j_{\beta-h+1}}$ by definition. $\varepsilon_{m}^{s, j_{h}}<0$ for $h<\alpha$ and, hence, the first sum is an integer. $(-1)^{\beta-h+1}+(-1)^{\beta+h}=0(1 \leqslant h \leqslant \beta-\alpha+1)$ and so the second sum is zero.

In the exceptional case,

$$
\begin{aligned}
\sum_{j=0}^{k+v} \frac{\alpha}{2^{\varepsilon_{m}^{s, j}}} & =\sum_{0 \leqslant j_{h} \leqslant k+v} \frac{(-1)^{h}}{2^{\varepsilon_{m}, j_{h}}} \\
& =\sum_{h=0}^{\alpha-1} \frac{(-1)^{h}}{2^{\varepsilon_{m}^{s, j_{h}}}}+\sum_{h=\alpha}^{\beta-2} \frac{(-1)^{h}}{2^{s, j_{h}}}+\frac{(-1)^{\beta-1}}{2^{v}}+\frac{(-1)^{\beta}}{2^{v}}+\sum_{h=\beta+1}^{2 \beta-\alpha-1} \frac{(-1)^{h}}{2^{\varepsilon_{m}^{s, j h}}} \\
& =\sum_{h=0}^{\alpha-1} \frac{(-1)^{h}}{2^{\varepsilon_{m}^{s, j_{h}}}}+\sum_{h=1}^{\beta-\alpha-1} \frac{(-1)^{\beta-h-1}}{2^{s, j_{\beta-h-1}}}+\sum_{h=1}^{\beta-\alpha-1} \frac{(-1)^{\beta+h}}{2^{\varepsilon_{m}, j_{\beta+h}}} \\
& =\sum_{h=0}^{\alpha-1} \frac{(-1)^{h}}{2^{\varepsilon_{m}^{s, j_{h}}}}+\sum_{h=1}^{\beta-\alpha-1} \frac{\left[(-1)^{\beta-h-1}+(-1)^{\beta+h}\right]}{2^{\varepsilon_{m}, j_{\beta+h}}}
\end{aligned}
$$

$\varepsilon_{m}^{s, j_{h}}<0$ for $h<\alpha$ and hence the 1st-sum is an integer. $(-1)^{\beta+h}+(-1)^{\beta-h-1}=$ $0(1 \leqslant h \leqslant \beta-\alpha-1)$ and hence the 2 nd-sum is zero.

Finally, the argument goes through in the case, $p=2$ and $m=2^{s+i-1}$ is the least element of $\mathcal{M}_{n, s}$ with obvious modifications for $j=i-1$.

PROPOSITION 5.2.3. Let $0 \leqslant s \leqslant r_{n}$. Then there exists an s-admissible $\beta$-sequence $\left\{\beta_{j}\right\}_{0 \leqslant j \leqslant r_{n, s}}$ such that (i) $0 \leqslant \beta_{j} \leqslant p-1$, (ii) $\beta_{0}=1$, (iii) $\beta_{j}=0$ if $j \notin$ $\{0\} \cup \bigcup[k+1, \ldots, k+\nu]$, (iv) if, either, $\Phi_{n, s}^{0}=\emptyset$ or $\varepsilon_{m}^{s, 0} \leqslant 0 \forall m \in \mathcal{M}_{n, s}$

$$
\begin{gathered}
t_{k}^{s}=p^{v} \Delta \in \Phi_{n, s}^{0} \\
v \geqslant k,(\Delta, p)=1
\end{gathered}
$$

then $\beta_{j}=0\left(1 \leqslant j \leqslant r_{n, s}\right)$. 
Proof. Let $0 \leqslant s \leqslant r_{n}$. The proof is by induction on elements $t_{k}^{s} \in \Phi_{n, s}^{0}$. Define $\beta_{0}=1$ and let $m^{0}=\sup \mathcal{M}_{n, s}, m^{0}=\sigma\left(t_{k_{0}}^{s}\right)$. If $k_{0}>0$ then put $\beta_{j}=0(0<$ $\left.j \leqslant k_{0}\right)$. Suppose $t_{k}^{s}=p^{v} \Delta \in \Phi_{n, s}^{0}(\nu \geqslant 1,(\Delta, p)=1)$ and assume that $\beta_{j}$ has been defined for $j \in[0, k]$. Assume that either $p \neq 2$ or $m=\sigma\left(t_{k}^{s}\right)$ is not the least element of $\mathcal{M}_{n, s}$. If there is no $j \in[0, k]$ such that $\varepsilon_{m}^{s, j}>0$ and $\beta_{j}>0$ then put $\beta_{j}=0(k+1 \leqslant j \leqslant k+v)$. If such a $j$ exists then let $j_{1}=\max \left\{j \in[0, k]: \beta_{j}>0\right.$ and $\left.\varepsilon_{m}^{s, j}>0\right\}$. Assume $\varepsilon_{m}^{s, j}>0$ for $j_{0} \leqslant j \leqslant j_{1}$. Except for the case, $p=2, j_{1}=k$, $\beta_{k-1}=\beta_{k}=1, t_{k-1}^{s}=2 t_{k}^{s}+1$; the sequence $\left\{\varepsilon_{m}^{s, j}\right\}_{j_{0} \leqslant j \leqslant j_{1}}$ is positive and strictly increasing and bounded above by $v$. Also, $\left\{\varepsilon_{m}^{s, j}\right\}_{k+1 \leqslant j \leqslant k+v}=(v v-1 \cdots 21)$. For $j \in[k+1, \ldots, k+v]$ put

$$
\beta_{j}= \begin{cases}0 & \text { if } k+1 \leqslant j<k+v+1-\varepsilon_{m}^{s, j_{1}}, \\ p-\beta_{j_{1}} & \text { if } \varepsilon_{m}^{s, j}=\varepsilon_{m}^{s, j_{1}}, \text { i.e. if } j=k+v+1-\varepsilon_{m}^{s, j_{1}}, \\ p-1-\beta_{j^{\prime}} & \text { if } \varepsilon_{m}^{s, j}=\varepsilon_{m}^{s, j^{\prime}}, \text { i.e. if } j=k+v+1-\varepsilon_{m}^{s, j^{\prime}} \text { for } j_{0} \leqslant j^{\prime}<j_{1}, \\ p-1 & \text { if } \varepsilon_{m}^{s, j} \neq \varepsilon_{m}^{s, j^{\prime}}, \text { i.e. if } j \neq k+v+1-\varepsilon_{m}^{s, j^{\prime}} \forall j_{0} \leqslant j^{\prime}<j_{1} .\end{cases}
$$

In the exceptional case, $p=2, j_{1}=k, \beta_{k-1}=\beta_{k}=1, t_{k-1}^{s}=2 t_{k}^{s}+1$, $\varepsilon_{m}^{s, k-1}=\varepsilon_{m}^{s, k}=v ;\left\{\varepsilon_{m}^{s, j}\right\}_{j_{0} \leqslant j \leqslant k-2}$ is a strictly increasing sequence bounded above by $(v-1)$ and $\left(\varepsilon_{m}^{s, j}\right)_{k+2 \leqslant j \leqslant k+v}=(v-1 v-2 \cdots 21)$. We put

$$
\beta_{j}= \begin{cases}1-\beta_{j^{\prime}} & \text { if } \varepsilon_{m}^{s, j}=\varepsilon_{m}^{s, j^{\prime}}, \text { i.e. if } j=k+v+1-\varepsilon_{m}^{s, j^{\prime}} \text { for } j_{0} \leqslant j^{\prime} \leqslant k-2, \\ 1 & \text { otherwise. }\end{cases}
$$

If $t_{l}^{s} \in \Phi_{n, s}^{0}$ is the consecutive element to $t_{k}^{s}(l>k+v)$ then put $\beta_{j}=0(k+$ $v<j \leqslant l$ ). Then it can, easily, be checked that the $\beta$-sequence is an $s$-admissible sequence.

Finally, if $p=2$ and $m$ is the least element of $\mathcal{M}_{n, s}, \beta_{j}$ is given by the above formulae for $j \leqslant i-1$ and

$$
\beta_{i}= \begin{cases}1-\beta_{i-1}+\beta_{j^{\prime}} & \text { if } \varepsilon_{m}^{s, j^{\prime}}=1 \text { for some } j_{0} \leqslant j^{\prime}<j_{1}, \\ 1-\beta_{i-1} & \text { if } \varepsilon_{m}^{s, j^{\prime}} \neq 1 \text { for all } j_{0} \leqslant j^{\prime}<j_{1} .\end{cases}
$$

\section{Relations in the Group $J_{p}\left(P_{n}(\mathbb{C})\right)$}

Let $0 \leqslant s \leqslant r_{n}$. The purpose of this section is to show that each $s$-admissible sequence gives rise to a relation in $J_{p}\left(P_{n}(\mathbb{C})\right)$ between $\psi_{\mathbb{R}}^{p^{s}}(\omega), \psi_{\mathbb{R}}^{p^{s+1}}(\omega), \cdots$. We shall thus obtain relations in $J_{p}\left(P_{n}(\mathbb{C})\right)$ corresponding to the $\alpha$-sequence of Proposition 5.2.2 and the $\beta$-sequence of Proposition 5.2.3.

\subsection{PRELIMINARIES}

We copy from $[1$, Section 2$]$ the definition of the number $m(t)$, i.e. 
DEFINITION 6.1.1. For $p$ odd,

$$
v_{p}(m(t))= \begin{cases}0 & \text { if } t \neq \equiv 0(\bmod (p-1)) \\ 1+v_{p}(t) & \text { if } t \equiv 0(\bmod (p-1)) .\end{cases}
$$

For $p=2$,

$$
v_{2}(m(t))= \begin{cases}1 & \text { if } t \neq \equiv 0(\bmod 2), \\ 2+v_{2}(t) & \text { if } t \equiv 0(\bmod 2) .\end{cases}
$$

Ref. [4] makes it possible to express the order $\left|J_{p}\left(P_{n}(\mathbb{C})\right)\right|$ of the group $J_{p}\left(P_{n}(\mathbb{C})\right)$ by means of the numbers $m(t)$. We shall now investigate the relation between the numbers $m(t)$ and the numbers $t_{0}^{s}=\left[n / p^{s}(p-1)\right]$ and hence prove that with the exception of the case $p=2, n \equiv 3(\bmod 4),\left|J_{p}\left(P_{n}(\mathbb{C})\right)\right|=p^{\sum_{s=0}^{r_{n}} t_{0}^{s}}$.

LEMMA 6.1.2. Let $p$ be odd. Then $\sum_{s=0}^{r_{n}}\left(\left[n / p^{s}(p-1)\right]-\left[(n-1) / p^{s}(p-1)\right]\right)=$ $v_{p}(m(n))$.

Proof. (i) If $n \not \equiv 0(\bmod (p-1))$ then $\left(\left[n / p^{s}(p-1)\right]=\left[(n-1) / p^{s}(p-1)\right]\right)$ $\left(1 \leqslant s \leqslant r_{n}\right)$. Also $v_{p}(m(n))=0$ by definition.

(ii) If $n \equiv 0(\bmod (p-1))$. Let $n=(p-1) p^{\alpha} \Delta,(\Delta, p)=1$. For $0 \leqslant s \leqslant \alpha$, $\left[n / p^{s}(p-1)\right]=\left[(p-1) p^{\alpha} \Delta / p^{s}(p-1)\right]=p^{\alpha-s} \Delta$ and

$$
\left[\frac{(n-1)}{p^{s}(p-1)}\right]=\left[p^{\alpha-s} \Delta-\frac{1}{p^{s}(p-1)}\right]=p^{\alpha-s} \Delta-1 \text {. }
$$

Thus, $\left[n / p^{s}(p-1)\right]-\left[n-1 / p^{s}(p-1)\right]=1$. For $s>\alpha,\left[n / p^{s}(p-1)\right]=$ $\left[\Delta / p^{s-\alpha}\right]=\left[(\Delta-1) / p^{s-\alpha}\right]$ since $s-\alpha>0$ and

$$
(\Delta, p)=1\left[\frac{(n-1)}{p^{s}(p-1)}\right]=\left[\frac{\Delta}{p^{s-\alpha}}-\frac{1}{p^{s}(p-1)}\right]
$$

and

$$
\begin{aligned}
& \frac{\Delta-1}{p^{s-\alpha}}<\frac{\Delta}{p^{s-\alpha}}-\frac{1}{(p-1) p^{s}}<\frac{\Delta}{p^{s-\alpha}} \\
& {\left[\frac{\Delta-1}{p^{s-\alpha}}\right]=\left[\frac{\Delta}{p^{s-\alpha}}-\frac{1}{(p-1) p^{s}}\right]=\left[\frac{\Delta}{p^{s-\alpha}}\right],}
\end{aligned}
$$

i.e. $\left[n / p^{s}(p-1)\right]=\left[(n-1) / p^{s}(p-1)\right]$. Thus,

$$
\begin{gathered}
\sum_{s}\left(\left[\frac{n}{p^{s}(p-1)}\right]-\left[\frac{(n-1)}{p^{s}(p-1)}\right]\right) \\
=\sum_{0 \leqslant s \leqslant \alpha} 1=1+\alpha=v_{p}(m(n)) .
\end{gathered}
$$

COROLLARY 6.1.3. Let $p$ be odd. Then $\sum_{s=0}^{r_{n}}\left[n / p^{s}(p-1)\right]=\sum_{n^{\prime}=0}^{n} v_{p}\left(m\left(n^{\prime}\right)\right)=$ $\sum_{v=0}^{[n / 2]} v_{p}(m(2 v))$. 
LEMMA 6.1.4. For $n$ even, $\sum_{s=0}^{r_{n}}\left(\left[n / 2^{s}\right]-\left[(n-2) / 2^{s}\right]\right)=v_{2}(m(n))=2+v_{2}(n)$. Proof. Along the same lines as that of Lemma 6.1.2 and the details are omitted.

COROLLARY 6.1.5. For $n$ even, $\sum_{s=0}^{r_{n}}\left[n / 2^{s}\right]=\sum_{v=1}^{n / 2} v_{2}(m(2 v))$.

COROLLARY 6.1.6. For $n \equiv 1(\bmod 4), \sum_{s=0}^{r_{n}}\left[n / 2^{s}\right]=1+\sum_{v=1}^{(1 / 2)(n-1)} v_{2}(m(2 v))$. Proof.

$$
\left[\frac{n}{2^{s}}\right]-\left[\frac{(n-1)}{2^{s}}\right]= \begin{cases}1 & \text { if } s=0, \\ 0 & \text { if } s \geqslant 1 .\end{cases}
$$

Thus, $\sum_{s=0}^{r_{n}}\left(\left[n / 2^{s}\right]-\left[(n-1) / 2^{s}\right]\right)=1$ and, hence,

$$
\sum_{s=0}^{r_{n}}\left[n / 2^{s}\right]=1+\sum_{s=0}^{r_{n}}\left[(n-1) / 2^{s}\right]=1+\sum_{v=1}^{(1 / 2)(n-1)} v_{2}(m(2 v))
$$

by Corollary 6.1.5.

PROPOSITION 6.1.7. Except for $p=2$ and $n \equiv 3(\bmod 4),\left|J_{p}\left(P_{n}(\mathbb{C})\right)\right|=$ $p^{\sum_{s=0}^{r_{n}} t_{0}^{s}}=p^{\sum_{s=0}^{r_{n}}\left[n / p^{s}(p-1)\right]}$.

Proof. By [4], there exist exact sequences,

(1) $0 \rightarrow \mathbb{Z}_{m(2 v)}=J\left(S^{4 v}\right) \rightarrow J\left(P_{2 v}(\mathbb{C})\right) \rightarrow J\left(P_{2 v-2}(\mathbb{C})\right) \rightarrow 0$.

(2) $0 \rightarrow \mathbb{Z}_{2}=J\left(S^{8 v+2}\right) \rightarrow J\left(P_{4 v+1}(\mathbb{C})\right) \rightarrow J\left(P_{4 v}(\mathbb{C})\right) \rightarrow 0$.

(3) $J\left(P_{4 v+3}(\mathbb{C})\right)=J\left(P_{4 v+2}(\mathbb{C})\right)$.

By continuous iteration of (1), we obtain (4)

(4) For $n$ even, $\left|J_{p}\left(P_{n}(\mathbb{C})\right)\right|=p^{\sum_{v=1}^{n / 2} v_{p}(m(2 v))}$.

If $p$ and $n$ are odd, $J_{p}\left(P_{n}(\mathbb{C})\right)=J_{p}\left(P_{n-1}(\mathbb{C})\right)$ and from (4) we obtain

(5) For $p$ and $n$ odd, $\left|J_{p}\left(P_{n}(\mathbb{C})\right)\right|=p^{\sum_{v=1}^{(1 / 2)(n-1)} v_{p}(m(2 v))}$.

Combining (4) and (5) for $p$ odd gives us

(6) For $p$ odd, $\left|J_{p}\left(P_{n}(\mathbb{C})\right)\right|=p^{\sum_{v=1}^{[n / 2]} v_{p}(m(2 v))}$.

The result for $p$ odd follows from (6) and Corollary 6.1.3. The result for $p=2$ and $n$ even follows from (4) and Corollary 6.1.5. We obtain from (2) and (4) the following:

(7) For $n \equiv 1(\bmod 4),\left|J_{2}\left(P_{n}(\mathbb{C})\right)\right|=2^{1+\sum_{v=1}^{(1 / 2)(n-1)} v_{2}(m(2 v))}$.

The result follows from this and Corollary 6.1.6.

DEFINITION 6.1.8. Let $0 \leqslant s \leqslant r_{n}$. We define

$$
\begin{aligned}
& \mathcal{J}(p, n, s)=\psi_{\mathbb{R}}^{p^{s}}\left(J_{p}\left(P_{n}(\mathbb{C})\right)\right) \\
& \quad=\text { Subgroup of } J_{p}\left(P_{n}(\mathbb{C})\right) \text { generated by } \psi_{\mathbb{R}}^{p^{s}}(\omega), \psi_{\mathbb{R}}^{p^{s+1}}(\omega), \ldots, \psi_{\mathbb{R}}^{p^{r_{n}}}(\omega) .
\end{aligned}
$$

PROPOSITION 6.1.9. The inclusion-map, $\iota: P_{4 v}(\mathbb{C}) \rightarrow P_{4 v+1}(\mathbb{C})$ induces an isomorphism, $\iota^{!}: \mathcal{J}(2,4 v+1, s) \stackrel{=}{\rightarrow} \mathcal{J}(2,4 v, s)$ for $s \geqslant 1$. 


\section{Proof.}

$$
\begin{aligned}
& J_{2}\left(P_{4 v+1}(\mathbb{C})\right)=\tilde{K}_{\mathbb{R}}\left(P_{4 v+1}(\mathbb{C})\right) / V_{4 v+1}, \\
& J_{2}\left(P_{4 v}(\mathbb{C})\right)=\tilde{K}_{\mathbb{R}}\left(P_{4 v}(\mathbb{C})\right) / V_{4 v}, V_{4 v+1}=\cap_{e} Y_{e},
\end{aligned}
$$

where $Y_{e}=\sum_{k \geqslant 1} \sum_{i=0}^{2 v+1} a_{k} 2^{e(k, i)}\left(\psi_{\mathbb{R}}^{2^{k}}-1\right) \omega^{i}, V_{4 v}=\cap_{e} Y_{e}^{\prime}$, where $Y_{e}^{\prime}=\sum_{k} \geqslant 1$ $\sum_{i=0}^{2 v} a_{k} 2^{e(k, i)}\left(\psi_{\mathbb{R}}^{2^{k}}-1\right) \omega^{i}, \psi_{\mathbb{R}}^{2}\left(\omega^{2 v+1}\right)=0$ and for $e(k, i) \geqslant 1,2^{e(k, 2 v+1)}\left(\psi_{\mathbb{R}}^{2^{k}}-\right.$ 1) $\omega^{2 v+1}=-2^{e(k, 2 v+1)} \omega^{2 v+1}=0$. Also, by Corollary 3.2.4, for $2^{k} \leqslant 4 v$, the coefficient of $\omega^{2 v+1}$ in the expansion of $\psi_{\mathbb{R}}^{2^{k}}(\omega)$ and, hence, of $\psi_{\mathbb{R}}^{2^{k}}\left(\omega^{i}\right),(0 \leqslant i \leqslant 2 v)$ is even. Thus, the element $\left(\psi_{\mathbb{R}}^{2^{k}}-1\right) \omega^{i} \in \tilde{K}_{\mathbb{R}}\left(P_{4 v+1}(\mathbb{C})\right)$, actually belongs to the subgroup, $\tilde{K}_{\mathbb{R}}\left(P_{4 v}(\mathbb{C})\right)$. Thus, $Y_{e}=Y_{e}^{\prime}$ and hence $V_{4 v+1}=V_{4 v} \cdot \mathcal{J}(2,4 v+1, s)=$ $\psi_{\mathbb{R}}^{2^{s}}\left(\tilde{K}_{\mathbb{R}}\left(P_{4 v+1}(\mathbb{C})\right)\right) / \psi_{\mathbb{R}}^{2^{s}}\left(V_{4 v+1}\right) \psi_{\mathbb{R}}^{2^{s}}\left(\tilde{K}_{\mathbb{R}}\left(P_{4 v+1}(\mathbb{C})\right)\right)=\left\{\psi_{\mathbb{R}}^{2^{s}}(\omega), \psi_{\mathbb{R}}^{2^{s}}\left(\omega^{2}\right), \ldots\right.$, $\left.\psi_{\mathbb{R}}^{2^{s}}\left(\omega^{2 v}\right), \psi_{\mathbb{R}}^{2^{s}}\left(\omega^{2 v+1}\right)\right\} \psi_{\mathbb{R}}^{2^{s}}\left(\omega^{2 v+1}\right)=0$ and the other elements belong to the subgroup $\tilde{K}_{\mathbb{R}}\left(P_{4 v}(\mathbb{C})\right)$.

Thus, $\psi_{\mathbb{R}}^{2^{s}}\left(\tilde{K}_{\mathbb{R}}\left(P_{4 v+1}(\mathbb{C})\right)\right)=\psi_{\mathbb{R}}^{2^{s}}\left(\tilde{K}_{\mathbb{R}}\left(P_{4 v}(\mathbb{C})\right)\right)$.

Hence, $\mathcal{J}(2,4 v+1, s)=\psi_{\mathbb{R}}^{2^{s}}\left(\tilde{K}_{\mathbb{R}}\left(P_{4 v}(\mathbb{C})\right)\right) / \psi^{2^{s}}\left(V_{4 v}\right)=\mathcal{J}(2,4 v, s)$.

\subsection{RELATIONS}

PROPOSITION 6.2.1. Let either $p$ be odd or $p=2$ and $n \not \equiv 1(\bmod 4), 0 \leqslant s \leqslant r_{n}$. Then $\sum_{j=0}^{r_{n, s}} \alpha_{j} p^{t_{j}^{s}} \psi_{\mathbb{R}}^{p^{s+j}}(\omega)=0$ in $J_{p}\left(P_{n}(\mathbb{C})\right)$ for $\alpha_{j} \in \mathbb{Z},\left(0 \leqslant j \leqslant r_{n, s}\right)$ if and only if $\left\{\alpha_{j}\right\}_{0 \leqslant j \leqslant r_{n, s}}$ is an $s$-admissible sequence.

Proof. By Proposition 2.2.1, $J_{p}\left(\sum_{j=0}^{r_{n}, s} \alpha_{j} p^{t_{j}^{s}} \psi_{\mathbb{R}}^{p^{s+j}}(\omega)\right)=0$ if and only if

$$
\rho_{\mathbb{R}}^{p}\left(\sum_{j=0}^{r_{n, s}} \alpha_{j} p^{t_{j}^{s}} \psi_{\mathbb{R}}^{p^{s+j}}(\omega)\right)=\prod_{j=0}^{r_{n, s}} \rho_{\mathbb{R}}^{p}\left(\psi_{\mathbb{R}}^{p^{s+j}}(\omega)\right)^{\alpha_{j} p^{t_{j}^{s}}} \in \mathbb{Z}
$$

Let

$$
\sum_{j=0}^{r_{n, s}} \rho_{\mathbb{R}}^{p}\left(\psi_{\mathbb{R}}^{p^{s+j}}(\omega)\right)^{\alpha_{j} p^{t_{j}^{s}}}=1+\sum_{m=1}^{[n / 2]} a_{m} \omega^{m} .
$$

By Corollary 4.3.11, if $m \notin \mathcal{M}_{n, s}$, then $a_{m} \in \mathbb{Z}$. Let $m \in \mathcal{M}_{n, s}, m=(1 / 2)(p-1)$ $p^{i} \Delta, i \geqslant 1,(\Delta, p)=1$. By Corollary 4.5.3, if either $p \neq 2$ or $m$ is not the least element of $\mathcal{M}_{n, s}, a_{m} \equiv \pm(1 / \Delta) \sum_{j=0}^{i}\left(\alpha_{j} / p^{\varepsilon_{m}^{s, j}}\right)\left(\bmod \hat{Q}_{p}\right)$. Since both $a_{m}$, $\sum_{j=0}^{i}\left(\alpha_{j} / p^{\varepsilon_{m}^{s, j}}\right) \in \mathbb{Q}_{p}$, either one of them belongs to $\mathbb{Z}$ if and only if it belongs to $\hat{Q}_{p}$. If $\sum_{j=0}^{i}\left(\alpha_{j} / p^{\varepsilon_{m}^{s, j}}\right) \in \hat{Q}_{p}$ then it follows from the congruence above and the fact that $(\triangle, p)=1$ that $a_{m} \in \hat{Q}_{p}$. Conversely, if $a_{m} \in \hat{Q}_{p}$ then $\sum_{j=0}^{i}\left(\alpha_{j} / p^{\varepsilon_{m}^{s, j}}\right) \equiv$ $\pm \triangle a_{m}\left(\bmod \hat{Q}_{p}\right)$ and thus, $\sum_{j=0}^{i}\left(\alpha_{j} / p^{\varepsilon_{m}^{s, j}}\right) \in \hat{Q}_{p}$. If $p=2$ and $m$ is the least 
element of $\mathcal{M}_{n, s}$, by similar argument, $a_{m} \in \mathbb{Z}$ if and only if $\sum_{\substack{0 \leqslant j \leqslant i \\ j \neq i-1}}\left(\alpha_{j} / 2^{\varepsilon_{m}^{s, j}}\right)-$ $\left(\alpha_{i-1} / 2^{\varepsilon_{m}^{s i-1}}\right) \in \hat{Q}_{2}$. Hence, $a_{m} \in \mathbb{Z} \forall 1 \leqslant m \leqslant[n / 2]$ if and only if $a_{m} \in \mathbb{Z}$ $\forall m \in \mathcal{M}_{n, s}$ if and only if $\sum_{j=0}^{i}\left(\alpha_{j} / p^{\varepsilon_{m}^{s, j}}\right) \in \mathbb{Z} \forall m \in \mathcal{M}_{n, s}$ except for $p=2$ and $m$ is the least element of $\mathcal{M}_{n, s}$, in which case, $\sum_{\substack{0 \leqslant j \leqslant i \\ j \neq i-1}}\left(\alpha_{j} / 2^{\varepsilon_{m}^{s, j}}\right)-\left(\alpha_{i-1} / 2^{\varepsilon_{m}^{s, i-1}}\right) \in \hat{Q}_{2}$, i.e. if and only if $\left\{\alpha_{j}\right\}_{0 \leqslant j \leqslant r_{n, s}}$ is an $s$-admissible sequence.

PROPOSITION 6.2.2. For either $p$ odd or $p=2$ and $n \not \equiv 1(\bmod 4)$ and for $0 \leqslant s \leqslant r_{n}$, there exists a relation, $\sum_{h \geqslant 0}(-1)^{h} p^{t_{j_{h}}^{s}} \psi_{\mathbb{R}}^{p^{s+j_{h}}}(\omega)=0$ in $J_{p}\left(P_{n}(\mathbb{C})\right)$ where $\left\{j_{h}\right\}_{h} \geqslant 0$ is the sequence constructed in Proposition 5.2.1.

Proof. It follows from Propositions 5.2.2 and 6.2.1.

In what follows, we shall drop $n$ and $p$ and simply write $\mathcal{J}_{s}$ for $\mathcal{J}(p, n, s)$.

PROPOSITION 6.2.3. Except for $p=2$ and $n \equiv 3(\bmod 4),\left|\mathcal{J}_{s} / \mathcal{J}_{s+1}\right|=p^{t_{0}^{s}}=$ $p^{\left[n / p^{s}(p-1)\right]}$.

Proof. (i) Let either $p$ be odd or $p=2$ and $n$ be even. We deduce from the relation given by Proposition 6.2.2 and the fact that $\alpha_{0}=1$ that $\left|\mathcal{J}_{s} / \mathcal{J}_{s+1}\right| / p^{t_{0}^{s}}$. It follows from Proposition 6.1.7 that $\prod_{s=0}^{r_{n}}\left|\mathcal{J}_{s} / \mathcal{J}_{s+1}\right|=\left|\mathcal{J}_{0}\right|=\left|J_{p}\left(P_{n}(\mathbb{C})\right)\right|=$ $\prod_{s=0}^{r_{n}} p^{t_{0}^{s}}$. Thus $\left|\mathcal{J}_{s} / \mathcal{J}_{s+1}\right|=p^{t_{0}^{s}}$.

(ii) Let $p=2$ and $n=4 v+1$. It follows from Proposition 6.1.9 and Part (i) of this proof that for $s \geqslant 1,\left|\mathcal{J}_{s} / \mathcal{J}_{s+1}\right|=2^{\left[4 v / 2^{s}\right]}=2^{\left[(4 v+1) / 2^{s}\right]}=2^{t_{0}^{s}}$.

$$
\begin{aligned}
\left|\frac{\mathcal{J}_{0}}{\mathcal{J}_{1}}\right| & =\frac{\left|\mathcal{J}_{0}\right|}{\left|\mathcal{J}_{1}\right|}=\frac{\left|J_{2}\left(P_{4 v+1}(\mathbb{C})\right)\right|}{\left|\mathcal{J}_{1}\right|}=\frac{\left|J_{2}\left(P_{4 v+1}(\mathbb{C})\right)\right|}{\left|J_{2}\left(P_{4 v}(\mathbb{C})\right)\right|} \frac{\left|J_{2}\left(P_{4 v}(\mathbb{C})\right)\right|}{\left|\mathcal{J}_{1}\right|} \\
& =2 \times 2^{4 v}=2^{4 v+1}=t_{0}^{0} .
\end{aligned}
$$

Let us recall the polynomial $T_{k}(x)$ defined in Definition 3.2.1 so that $\psi_{\mathbb{R}}^{k}(\omega)=T_{k}(\omega)$ (e.g. Proposition 3.2.2). We now state a recursion relation for $T_{k}(x)$.

LEMMA 6.2.4. $T_{k+1}(x)-(x+2) T_{k}(x)+T_{k-1}(x)=2 x$.

Proof. This is [10, Theorem 5.2.3].

COROLLARY 6.2.5. -4 is a root of

$T_{k}(x)$ if $k$ is even, $\quad T_{k}(x)-x$ if $k$ is odd.

Proof. This follows by induction from Lemma 6.2.4 and the fact that $T_{1}(x)=x$ and $T_{2}(x)=4 x+x^{2}$.

LEMMA 6.2.6. If in $J_{2}\left(P_{2 v}(\mathbb{C})\right)$, we express $\omega^{v}=\sum_{s=0}^{r_{2 v}} \beta_{s} \psi_{\mathbb{R}}^{2 s}(\omega)$ then $\beta_{0}=$ $(-4)^{v-1}=(-1)^{v-1} 2^{2 v-2}$. 
Proof. It is by induction on $v$. It is true for $v=1$. Let $v>1$ and assume it to be true for $v^{\prime}<v$.

(i) Let $v$ be even. $\psi_{\mathbb{R}}^{v}(\omega)=T_{v}(\omega)=\sum_{k<v} a_{k} \omega^{k}+\omega^{v}$. Let $v=2^{l} v^{\prime}, l \geqslant 1, v^{\prime}$ odd. Then $\psi_{\mathbb{R}}^{v}-\psi_{\mathbb{R}}^{2^{l}}=\psi_{\mathbb{R}}^{2^{l}} \circ\left(\psi_{\mathbb{R}}^{v^{\prime}}-1\right)$ and $\psi_{\mathbb{R}}^{v^{\prime}}-1=0$ on $J_{2}\left(P_{2 v}(\mathbb{C})\right)$ by [12, Proposition 2.3.3]. Thus, $\psi_{\mathbb{R}}^{v}(\omega)=\psi_{\mathbb{R}}^{2^{l}}(\omega)$. Hence, $\omega^{v}=-\sum_{k<v} a_{k} \omega^{k}+\psi_{\mathbb{R}}^{2^{l}}(\omega)$, $\omega^{k}=\sum_{s=0}^{r_{2 v}} \beta_{s}^{k} \psi_{\mathbb{R}}^{2^{s}}(\omega)$. and $\omega^{v}=-\sum_{k<v} a_{k}\left(\sum_{s=0}^{r_{2 v}} \beta_{s}^{k} \psi_{\mathbb{R}}^{2^{s}}(\omega)\right)+\psi_{\mathbb{R}}^{2^{l}}(\omega)$. By the induction-hypothesis, $\beta_{0}^{k}=(-4)^{k-1}$ and thus, $\beta_{0}=-\sum_{k<v} a_{k}(-4)^{k-1}$. It follows from Corollary 6.2.5 that $0=T_{v}(-4)=\sum_{k<v}(-4)^{k} a_{k}+(-4)^{v}$ and thus, $\sum_{k<v}(-4)^{k} a_{k}=(-4)^{v}$ and dividing by -4 we obtain $\sum_{k<v}(-4)^{k-1} a_{k}=$ $-(-4)^{v-1}$. Hence, $\beta_{0}=(-4)^{v-1}$.

(ii) Let $v$ be odd. Then $\omega=\psi_{\mathbb{R}}^{v}(\omega)=T_{v}(\omega)=\sum_{k<v} a_{k} \omega^{k}+\omega^{v}$.

$$
\begin{aligned}
\omega^{v} & =\omega-\sum_{k<v} a_{k} \omega^{k} . \\
\beta_{0} & =1-\sum_{k<v} a_{k} \beta_{0}^{k}=1-\sum_{k<v} a_{k}(-4)^{k-1} . \\
0 & =T_{v}(-4)+4=\sum_{k<v} a_{k}(-4)^{k}+(-4)^{v}+4, \\
1 & -\sum_{k<v} a_{k}(-4)^{k-1}=(-4)^{v-1} .
\end{aligned}
$$

Hence $\beta_{0}=(-4)^{v-1}$.

We shall now waive the unnecessary restriction in the hypothesis of Proposition 6.2.1 and prove Proposition 6.2.1 without any restrictions on $n$ and $p$.

PROPOSITION 6.2.7. Let $0 \leqslant s \leqslant r_{n}$. Then $\sum_{j=0}^{r_{n, s}} \alpha_{j} p^{t_{j}^{s}} \psi_{\mathbb{R}}^{p^{s+j}}(\omega)=0$ in $J_{p}$ $\left(P_{n}(\mathbb{C})\right)$ for $\alpha_{j} \in \mathbb{Z}\left(0 \leqslant j \leqslant r_{n, s}\right)$ if and only if $\left\{\alpha_{j}\right\}_{0 \leqslant j \leqslant r_{n, s}}$ is an s-admissible sequence.

Proof. In view of Proposition 6.2.1, it suffices to restrict attention to $p=2$ and $n=4 v+1$. For $s \geqslant 1, \Phi_{4 v+1, s}=\Phi_{4 v, s}$ and, hence, $s$-admissible sequences for $P_{4 v+1}(\mathbb{C})$ and those for $P_{4 v}(\mathbb{C})$ are in 1-1 correspondence. For $s=0, \Phi_{4 v+1}=$ $\left(4 v+1,2 v, t_{2}, t_{3}, \ldots\right)$ and $\Phi_{4 v}=\left(4 v, 2 v-1, t_{2}, t_{3}, \ldots\right)$, where $t_{2}=v-1$. Obviously, $\mathcal{M}_{4 v+1}=\mathcal{M}_{4 v}$. Suppose $\alpha_{0} 2^{4 v+1} \omega+\alpha_{1} 2^{2 v} \psi_{\mathbb{R}}^{2}(\omega)+\sum_{j \geqslant 2} \alpha_{j} 2^{t_{j}} \psi_{\mathbb{R}}^{2^{j}}(\omega)=0$ is a relation in $J_{2}\left(P_{4 v+1}(\mathbb{C})\right)$. Then $\left(2 \alpha_{0}\right) 2^{4 v} \omega+\left(2 \alpha_{1}\right) 2^{2 v-1} \psi_{\mathbb{R}}^{2}(\omega)+\sum_{j \geqslant 2} \alpha_{j} 2^{t_{j}} \psi_{\mathbb{R}}^{2^{j}}$ $(\omega)=0$ in $J_{2}\left(P_{4 v}(\mathbb{C})\right)$ by naturality. It follows from Proposition 6.2.1 that $\left(2 \alpha_{0}, 2 \alpha_{1}\right.$, $\left.\alpha_{2}, \alpha_{3}, \ldots\right)$ is an admissible sequence for $P_{4 v}(\mathbb{C})$. Let $m \in \mathcal{M}_{4 v+1}=\mathcal{M}_{4 v}$ and let $\varepsilon_{m}^{j}$ and $\bar{\varepsilon}_{m}^{j}$ denote the $j$-indices of $m$ for $P_{4 v+1}(\mathbb{C})$ and $P_{4 v}(\mathbb{C})$, respectively. Then $\varepsilon_{m}^{0}=\bar{\varepsilon}_{m}^{0}-1, \varepsilon_{m}^{1}=\bar{\varepsilon}_{m}^{1}-1, \varepsilon_{m}^{j}=\bar{\varepsilon}_{m}^{j}(j \geqslant 2)$. If $m$ is not the least element of $\mathcal{M}_{4 v+1}$ then

$$
\frac{2 \alpha_{0}}{2^{\bar{\varepsilon}_{m}^{0}}}+\frac{2 \alpha_{1}}{2^{\bar{\varepsilon}_{m}^{1}}}+\sum_{j \geqslant 2} \frac{\alpha_{j}}{2^{\bar{\varepsilon}_{m}^{j}}} \in \mathbb{Z}, \text { i.e. } \frac{\alpha_{0}}{2^{\varepsilon_{m}^{0}}}+\frac{\alpha_{1}}{2^{\varepsilon_{m}^{1}}}+\sum_{j \geqslant 2} \frac{\alpha_{j}}{2^{\varepsilon_{m}^{j}}} \in \mathbb{Z},
$$


and if $m$ is not least element of $\mathcal{M}_{4 v+1}$ then

$$
\frac{2 \alpha_{0}}{2^{\bar{\varepsilon}_{m}^{0}}}+\frac{2 \alpha_{1}}{2^{\bar{\varepsilon}_{m}^{1}}}+\sum_{\substack{j \geqslant 2 \\ j \neq i-1}} \frac{\alpha_{j}}{2^{\bar{\varepsilon}_{m}^{j}}}-\frac{\alpha_{i-1}}{2^{\bar{\varepsilon}_{m}^{i-1}}} \in \mathbb{Z} ;
$$

i.e.

$$
\frac{2 \alpha_{0}}{2^{\varepsilon_{m}^{0}}}+\frac{2 \alpha_{1}}{2^{\varepsilon_{m}^{1}}}+\sum_{\substack{j \geqslant 2 \\ j \neq i-1}} \frac{\alpha_{j}}{2^{\varepsilon_{m}^{j}}}-\frac{\alpha_{i-1}}{2^{\varepsilon_{m}^{i-1}}} \in \mathbb{Z} .
$$

Hence $\left(\alpha_{0}, \alpha_{1}, \alpha_{2}, \ldots\right)$ is an admissible sequence for $P_{4 v+1}(\mathbb{C})$.

Conversely, let $\left\{\alpha_{j}\right\}$ be an admissible sequence for $P_{4 v+1}(\mathbb{C})$. As in the above, $\left(2 \alpha_{0}, 2 \alpha_{1}, \alpha_{2}, \ldots\right)$ is an admissible sequence for $P_{4 v}(\mathbb{C})$ and by Proposition 6.2.1, $\left(2 \alpha_{0}\right) 2^{4 v} \omega+\left(2 \alpha_{1}\right) 2^{2 v-1} \psi_{\mathbb{R}}^{2}(\omega)+\sum_{j \geqslant 2} \alpha_{j} 2^{t_{j}} \psi_{\mathbb{R}}^{2^{j}}(\omega)=0$, i.e. $\alpha_{0} 2^{4 v+1} \omega+\alpha_{1} 2^{2 v} \psi_{\mathbb{R}}^{2}$ $(\omega)+\sum_{j \geqslant 2} \alpha_{j} 2^{t_{j}} \psi_{\mathbb{R}}^{2^{j}}(\omega)=0$ in $J_{2}\left(P_{4 v}(\mathbb{C})\right)$. From the exactness of the sequence, $0 \rightarrow J\left(P_{4 v+2}(\mathbb{C}) / P_{4 v}(\mathbb{C})\right) \rightarrow J\left(P_{4 v+2}(\mathbb{C})\right) \rightarrow J\left(P_{4 v}(\mathbb{C})\right) \rightarrow 0$ proved in [4], we conclude that in $J_{2}\left(P_{4 v+2}(\mathbb{C})\right)$,

$$
\begin{aligned}
& \alpha_{0} 2^{4 v+1} \omega+\alpha_{1} 2^{2 v} \psi_{\mathbb{R}}^{2}(\omega)+\sum_{j \geqslant 2} \alpha_{j} 2^{t_{j}} \psi_{\mathbb{R}}^{2^{j}}(\omega)=\beta \omega^{2 v+1} \\
& =\beta\left(2^{4 v} \omega+\sum_{s \geqslant 1} \beta_{s} \psi_{\mathbb{R}}^{2^{s}}(\omega)\right) .
\end{aligned}
$$

By Lemma 6.2.6, $\left(\alpha_{0} 2^{4 v+1}-\beta 2^{4 v}\right) \omega+\sum_{s} \geqslant 1 \gamma_{s} \psi_{\mathbb{R}}^{2^{s}}(\omega)=0$. By Proposition 6.2.3, $2^{4 v+2} \mid\left(\alpha_{0} 2^{4 v+1}-\beta 2^{4 v}\right)$. Thus $\beta$ is even. If $\iota: P_{4 v+1}(\mathbb{C}) \subset P_{4 v+2}(\mathbb{C})$ is the inclusionmap, we apply $\iota$ ' to Equation (6.1) and hence obtain

$$
\alpha_{0} 2^{4 v+1} \omega+\alpha_{1} 2^{2 v} \psi_{\mathbb{R}}^{2}(\omega)+\sum_{j \geqslant 2} \alpha_{j} 2^{t_{j}} \psi_{\mathbb{R}}^{2^{j}}(\omega)=0 \quad \text { in } J_{2}\left(P_{4 v+1}(\mathbb{C})\right) .
$$

PROPOSITION 6.2.8. There exist $\left(r_{n}+1\right)$-relations, $\sum_{h \geqslant 0}(-1)^{\varepsilon(h)} p^{t_{j h}^{s}} \psi_{\mathbb{R}}^{p^{s+j_{h}}}$ $(\omega)=0\left(0 \leqslant s \leqslant r_{n}\right)$ in $J_{p}\left(P_{n}(\mathbb{C})\right)$, where $\left\{j_{h}\right\}_{h} \geqslant 0$ is the sequence constructed in Proposition 5.2.1 where

$$
\varepsilon(h)= \begin{cases}h & \begin{array}{l}
\text { if either } p \neq 2 \text { or } p=2 \text { and } j_{h} \neq i-1 \text { where } m=2^{s+i-1} \\
\text { is the least element of } \mathcal{M}_{n, s}
\end{array} \\
h+1 & \begin{array}{l}
\text { if } p=2, m=2^{s+i-1} \\
\text { and } j_{h}=i-1,
\end{array}\end{cases}
$$

and this relation is complete except for $p=2$ and $n \equiv 3(\bmod 4)$.

Proof. The existence of the relation follows from Propositions 5.2.2 and 6.2.7 and completeness from Proposition 6.2.3.

From now onwards we shall refer to this relation as the $\alpha$-relation. 
PROPOSITION 6.2.9. There exist $\left(r_{n}+1\right)$-relations, $\sum_{j=0}^{r_{n . s}} \beta_{j} p^{t_{j}^{s}} \psi_{\mathbb{R}}^{p^{s+j}}(\omega)=0$ $\left(0 \leqslant s \leqslant r_{n}\right)$ in $J_{p}\left(P_{n}(\mathbb{C})\right)\left(0 \leqslant s \leqslant r_{n}\right)$ where $\left\{\beta_{j}\right\}_{0 \leqslant j \leqslant r_{n . s}}$ is the $\beta$-sequence defined in Proposition 5.2.3 and this relation is complete except for $p=2$ and $n \equiv 3$ $(\bmod 4)$.

Proof. The existence of the relation follows from Propositions 5.2.3 and 6.2.7 and completeness from Proposition 6.2.3.

From now onwards we shall refer to this relation as the $\beta$-relation.

As a corollary of the $\alpha$-relations, we relate $\mathcal{J}(p, n, k)$ defined in Definition 6.1.8 to the $J_{p}$-group of a suitable complex projective space from which Proposition 3.3.1 follows as a corollary.

PROPOSITION 6.2.10. Let $0 \leqslant s \leqslant r_{n}$. Then $\mathcal{J}(p, n, k)=J_{p}\left(P_{\left[n / p^{k}\right]}(\mathbb{C})\right)$.

Proof. For $P_{n}(\mathbb{C}), t_{0}^{k+i}=\left[n /(p-1) p^{k+i}\right]$ and for $P_{\left[n / p^{k}\right]}(\mathbb{C}), t_{0}^{i}=$ $\left[n /(p-1) p^{k+i}\right]\left(0 \leqslant i \leqslant r_{n}-k\right)$, i.e. they are equal. Hence $\Phi_{n, k+i}=\Phi_{\left[n / p^{k}\right], i}$ and thus the $i$ th $\alpha$-relation for $\mathcal{J}(p, n, k)$ satisfied by $\psi_{\mathbb{R}}^{p^{k}}(\omega), \psi_{\mathbb{R}}^{p^{k+1}}(\omega), \ldots$ which is the $(k+i)$ th $\alpha$-relation for $J_{p}\left(P_{n}(\mathbb{C})\right)$ has the same coefficients as the $i$ th $\alpha$-relation for $J_{\left[n / p^{k}\right]}(\mathbb{C})$ satisfied by $\omega, \psi_{\mathbb{R}}^{p}(\omega), \ldots$. Hence $\mathcal{J}(p, n, k)=$ $J_{p}\left(P_{\left[n / p^{k}\right]}(\mathbb{C})\right)$.

Proof (Alternative proof of Proposition 3.3.1.). By Proposition 6.2.10 J $(p, n$, $k)=J_{p}\left(P_{\left[n / p^{k}\right]}(\mathbb{C})\right)$ and $\psi_{\mathbb{R}^{p^{k}}}^{p^{k}}(\omega) \in \mathcal{J}(p, n, k)$ corresponds to $\omega \in J_{p}\left(P_{\left[n / p^{k}\right]}\right.$ $(\mathbb{C}))$. Hence, the order of $\psi_{\mathbb{R}}^{p^{k}}(\omega)$ in $J_{p}\left(P_{n}(\mathbb{C})\right)$ or, equivalently, in $\mathcal{J}(p, n, k)$ is equal to the order of $\omega$ in $J_{p}\left(P_{\left[n / p^{k}\right]}(\mathbb{C})\right)$ which is equal to $M_{\left[n / p^{k}\right]+1, p}$ by the results of [4] and [5].

PROPOSITION 6.2.11. Let $n=p^{k}(p-1)+r(0 \leqslant r \leqslant p-2)$. Then $J_{p}\left(P_{n}(\mathbb{C})\right)$ is generated by $\left\{\omega, \psi_{\mathbb{R}}^{p}(\omega), \ldots, \psi_{\mathbb{R}}^{p^{k}}(\omega)\right\}$ subject to the relations,

$$
\begin{aligned}
& p^{p^{k}} \omega=p^{\left(p^{k-1}-1\right)} \psi_{\mathbb{R}}^{p}(\omega), \quad p^{p^{k-1}} \psi_{\mathbb{R}}^{p}(\omega)=p^{\left(p^{k-2}-1\right)} \psi_{\mathbb{R}}^{p^{2}}(\omega), \ldots, \\
& p^{p^{2}} \psi_{\mathbb{R}}^{p^{k-2}}(\omega)=(-1)^{p-1} p^{p-1} \psi_{\mathbb{R}}^{p^{k-1}}(\omega), \\
& p^{p} \psi_{\mathbb{R}}^{p^{k-1}}(\omega)=\psi_{\mathbb{R}}^{p^{k}}(\omega), \quad p \psi_{\mathbb{R}}^{p^{k}}(\omega)=0 .
\end{aligned}
$$

Proof.

$$
\begin{aligned}
& {[n /(p-1)]=p^{k} \text { and } t_{0}^{s}=\left[n / p^{s}(p-1)\right]=p^{k-s} .} \\
& \Phi_{n, s}=\left(p^{k-s},\left(p^{k-s-1}-1\right),\left(p^{k-s-2}-1\right), \ldots, 0\right), \Phi_{n, s}^{0}=\left(p^{k-s}\right), \\
& \mathcal{M}_{n, s}=\left((1 / 2)(p-1) p^{k}\right)
\end{aligned}
$$

and $\varepsilon_{(1 / 2)(p-1) p^{k}}^{s, 0}=k-s$ and $\varepsilon_{(1 / 2)(p-1) p^{k}}^{s, 1}=k-s$. Hence, the $s$ th $\alpha$-relation reads as: $p^{p^{k-s}} \psi_{\mathbb{R}}^{p^{s}}(\omega)-p^{\left(p^{k-s-1}-1\right)} \psi_{\mathbb{R}}^{p^{s+1}}=0(0 \leqslant s \leqslant k)$ if either $p \neq 2$ or $p=2$ and 
$s \neq k-2$ and $2^{4} \psi_{\mathbb{R}}^{2^{k-2}}(\omega)+2^{1} \psi_{\mathbb{R}}^{p^{k-1}}(\omega)=0$ if $p=2$ and $s=k-2$ and the result follows from Proposition 6.2.8.

PROPOSITION 6.2.12. Let $n=p^{k}(p-1)+r(0 \leqslant r \leqslant p-2)$. Then

$$
J_{p}\left(P_{n}(\mathbb{C})\right)=\mathbb{Z}_{p^{\left(p^{k}+k\right)}} \oplus \mathbb{Z}_{p^{\left(p^{k-1}-1\right)}} \oplus \mathbb{Z}_{p^{\left(p^{k-2}-1\right)}} \oplus \cdots \oplus \mathbb{Z}_{p^{(p-1)}} .
$$

Proof. Let $x_{0}=\omega, x_{1}=\psi_{\mathbb{R}}^{p}(\omega)-p^{\left(p^{k}-p^{k-1}+1\right)} \omega, x_{2}=\psi_{\mathbb{R}}^{p^{2}}(\omega)-p^{\left(p^{k-1}-p^{k-2}+1\right)}$ $\psi_{\mathbb{R}}^{p}(\omega), \ldots, x_{k-1}=\psi_{\mathbb{R}}^{p^{k-1}}(\omega)-(-1)^{p-1} p^{\left(p^{2}-p+1\right)} \psi_{\mathbb{R}}^{p^{k-2}}(\omega)$. It follows from the relations of Proposition 6.2 .11 that $\left\{x_{0}, x_{1}, \ldots, x_{k-1}\right\}$ are linearly independent and span $J_{p}\left(P_{n}(\mathbb{C})\right)$, i.e. they form a basis for $J_{p}\left(P_{n}(\mathbb{C})\right)$. Their orders also follow in a straightforward way from the $J$-relations of Proposition 6.2.11. The order of $x_{0}$ is the $p$-component of the Atiyah-Todd number $M_{n+1}$ which is equal to $p^{p^{k}+p}$ by the results of [4] and [5].

EXAMPLE 6.2.13. As a demonstration, we shall write down the $\alpha$ - and $\beta$ relations for $p=2$ and $n=164$.

$$
\begin{aligned}
& \Phi_{164,0}=\left\{\begin{array}{llllllll}
164 & 81 & 40 & 19 & 9 & 4 & 1 & 0
\end{array}\right\} \\
& \begin{array}{lllllllll}
\varepsilon_{82}^{0, j} & 2 & 2 & 1 & & & & & \\
\varepsilon_{80}^{0, j} & 1 & 3 & 3 & 3 & 2 & 1 & & \\
\varepsilon_{64}^{0, j} & & & & & 1 & 2 & 2 & 2
\end{array}
\end{aligned}
$$

$$
\begin{gathered}
\alpha \text {-relation : } 2^{164} \omega-2^{81} \psi_{\mathbb{R}}^{2}(\omega)+2^{19} \psi_{\mathbb{R}}^{8}(\omega)-2^{4} \psi_{\mathbb{R}}^{32}(\omega)-2^{1} \psi_{\mathbb{R}}^{64}(\omega)- \\
-\psi_{\mathbb{R}}^{128}(\omega)=0
\end{gathered}
$$

$\beta$-relation : $2^{164} \omega+2^{81} \psi_{\mathbb{R}}^{2}(\omega)+2^{40} \psi_{\mathbb{R}}^{4}(\omega)+2^{9} \psi_{\mathbb{R}}^{16}(\omega)+2^{1} \psi_{\mathbb{R}}^{64}(\omega)=0$.

$$
\begin{aligned}
& \Phi_{164,1}=\left\{\begin{array}{lllllll}
82 & 40 & 19 & 9 & 4 & 1 & 0
\end{array}\right\} \\
& \begin{array}{lllllllll}
\varepsilon_{82}^{1, j} & 1 & 1 & & & & & \\
\varepsilon_{80}^{1, j} & & 2 & 3 & 3 & 2 & 1 & & \\
\varepsilon_{64}^{1, j} & & & & 1 & 2 & 2 & 2 & 1
\end{array}
\end{aligned}
$$

$\alpha$-relation $: 2^{82} \psi_{\mathbb{R}}^{2}(\omega)-2^{40} \psi_{\mathbb{R}}^{4}(\omega)+2^{19} \psi_{\mathbb{R}}^{8}(\omega)-2^{9} \psi_{\mathbb{R}}^{16}(\omega)-2^{1} \psi_{\mathbb{R}}^{64}(\omega)-$

$$
-\psi_{\mathbb{R}}^{128}(\omega)=0
$$

$\beta$-relation : $2^{82} \psi_{\mathbb{R}}^{2}(\omega)+2^{40} \psi_{\mathbb{R}}^{4}(\omega)+2^{19} \psi_{\mathbb{R}}^{8}(\omega)+2^{4} \psi_{\mathbb{R}}^{32}(\omega)+2^{1} \psi_{\mathbb{R}}^{64}(\omega)+$

$$
+\psi_{\mathbb{R}}^{128}(\omega)=0 \text {. }
$$

$$
\begin{aligned}
& \Phi_{164,2}=\left\{\begin{array}{llllll}
41 & 20 & 9 & 4 & 1 & 0
\end{array}\right\} \\
& \begin{array}{lllllllll}
\varepsilon_{80}^{2, j} & : & & 2 & 2 & 2 & 1 & & \\
\varepsilon_{64}^{2, j} & : & & & & 2 & 2 & 2 & 1
\end{array}
\end{aligned}
$$

$\alpha$-relation $: 2^{41} \psi_{\mathbb{R}}^{4}(\omega)-2^{9} \psi_{\mathbb{R}}^{16}(\omega)-2^{1} \psi_{\mathbb{R}}^{64}(\omega)=0$

$\beta$-relation : $2^{41} \psi_{\mathbb{R}}^{4}(\omega)+2^{9} \psi_{\mathbb{R}}^{16}(\omega)+2^{4} \psi_{\mathbb{R}}^{32}(\omega)+\psi_{\mathbb{R}}^{128}(\omega)=0$. 


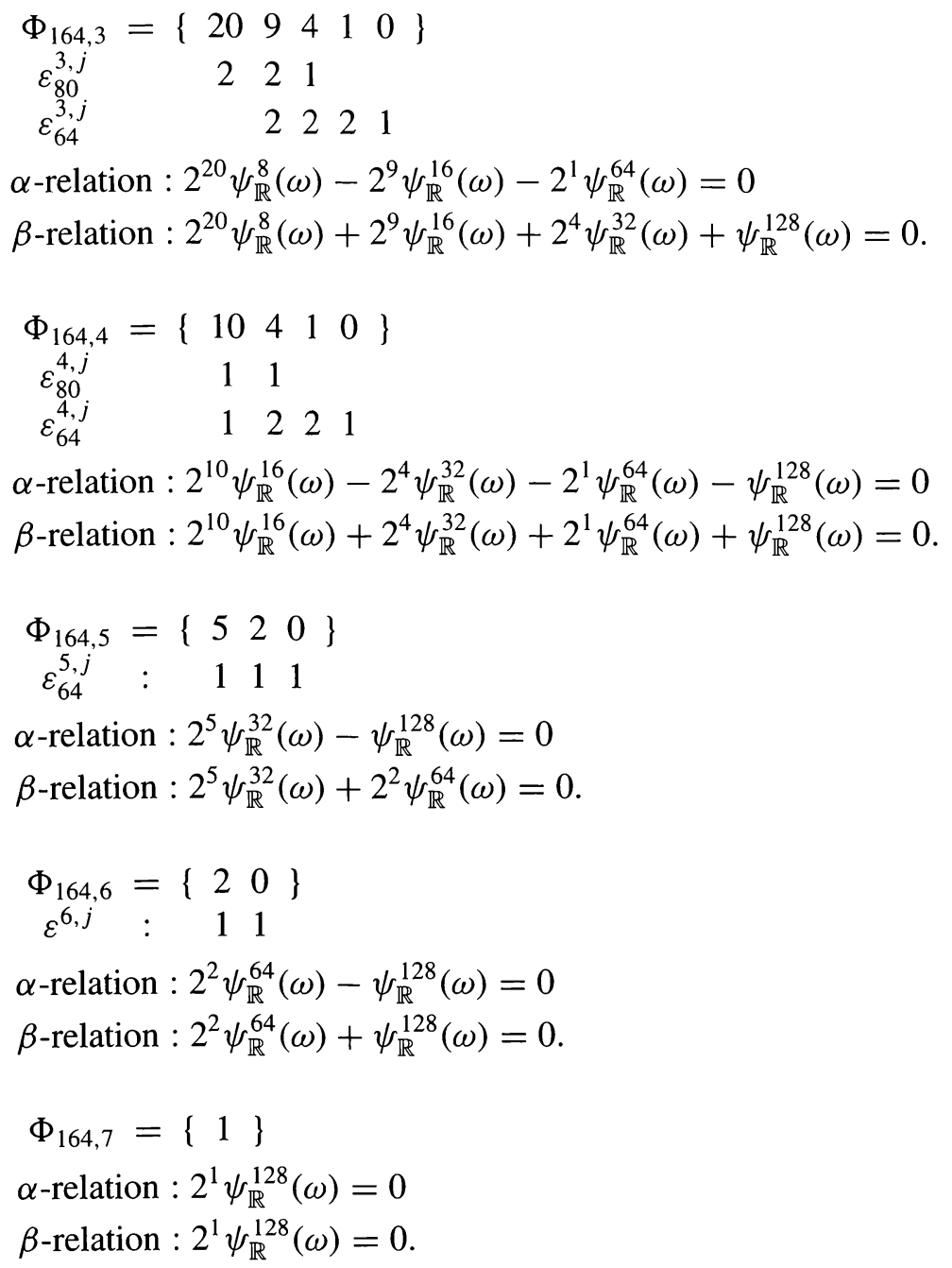

PROPOSITION 6.2.14. Let $n \in \mathbb{Z}^{+}, 0 \leqslant s \leqslant r_{n}$. Except for the case $p=2$ and $n \equiv 3(\bmod 4), \sum_{j=0}^{r_{n, s}} \alpha_{j}^{\prime} \psi_{\mathbb{R}}^{p^{s+j}}(\omega)=0$ in $J_{p}\left(P_{n}(\mathbb{C})\right)$ for $\alpha_{j}^{\prime} \in \mathbb{Z}$ if and only if $\alpha_{j}^{\prime}=p^{t_{j}^{s}} \alpha_{j}\left(0 \leqslant j \leqslant r_{n, s}\right)$ for some s-admissible sequence $\left\{\alpha_{j}\right\}_{0} \leqslant j \leqslant r_{n, s}$.

Proof. Let $0 \leqslant s \leqslant r_{n}$. In view of Proposition 6.2.7, it suffices to prove that if $\sum_{j=0}^{r_{n, s}} \alpha_{j}^{\prime} \psi_{\mathbb{R}}^{p^{s+j}}(\omega)=0$ in $J_{p}\left(P_{n}(\mathbb{C})\right)$ then $\alpha_{j}^{\prime}=p^{t_{j}^{s}} \alpha_{j}$ for $\alpha_{j} \in \mathbb{Z}\left(0 \leqslant j \leqslant r_{n, s}\right)$. Let Equation $s$ be $\sum_{j=0}^{r_{n, s}} \alpha_{j}^{\prime} \psi_{\mathbb{R}}^{p^{s+j}}(\omega)=0$. For $s \leqslant k \leqslant r_{n}$, let $\left\{\gamma_{j}^{k}\right\}_{0 \leqslant j \leqslant r_{n, s}}$ be a $k$-admissible sequence such that $\gamma_{0}^{k}=1$ whose existence follows from either Proposition 5.2.2 or Proposition 5.2.3. By Proposition 6.2.7, $\sum_{j=0}^{r_{n, s}} \gamma_{j}^{k} p^{t_{j}^{k}} \psi_{\mathbb{R}}^{p^{k+j}}$ $(\omega)=0$ in $J_{p}\left(P_{n}(\mathbb{C})\right)$. Call this equation $k^{\prime}$. For $s \leqslant k \leqslant r_{n}$, we claim the following statements: (i) $\alpha_{j}^{\prime}=p^{t_{j}^{s}} \alpha_{j}(0 \leqslant j \leqslant k-s)$ and (ii) there exists an equation $k$ of the form: $\sum_{j=k-s}^{r_{n}}\left(\alpha_{j}^{\prime}-p^{t_{j}^{s}} \triangle_{j}\right) \psi_{\mathbb{R}}^{p^{s+j}}(\omega)=0$. Proof. It is by induction on 
$k$. For $k=s$, it follows from Proposition 6.2.3 and Equation $s$ that $p^{t_{0}^{s}} \mid \alpha_{0}^{\prime}$, i.e. $\alpha_{0}^{\prime}=p^{t_{0}^{s}} \mid \alpha_{0}$ for some $\alpha_{0} \in \mathbb{Z}$. Let $k \geqslant 1$ and assume the induction-hypothesis for $(k-1)$. Equation $(k-1)$ is of the form: $\sum_{j=k-1-s}^{r_{n . s}}\left(\alpha_{j}^{\prime}-p^{t_{j}^{s}} \triangle_{j}\right) \psi_{\mathbb{R}}^{p^{s+j}}(\omega)=$ 0 . By the induction-hypothesis, $p^{t_{k-1-s}^{s}} \mid \alpha_{k-1-s}^{\prime}$. Let $\alpha_{k-1-s}^{\prime}=p^{t_{k-1-s}^{s}} \alpha_{k-1-s}$ for some $\alpha_{k-1-s} \in \mathbb{Z}$. The coefficient of $\psi_{\mathbb{R}}^{p^{k-1}}(\omega)$ is $\left(\alpha_{k-1-s}^{\prime}-p^{t_{k-1-s}^{s}} \delta_{k-1-s}\right)=$ $p^{t_{k-1-s}^{s}} \alpha_{k-1-s}-p^{t_{k-1-s}^{s}} \delta_{k-1-s}=p^{t_{k-1-s}^{s}}\left(\alpha_{k-1-s}-\delta_{k-1-s}\right)=\theta p^{t_{k-1-s}^{s}}$. We subtract $\theta$ times Equation $(k-1)^{\prime}$ from Equation $(k-1)$ to eliminate the term $\psi_{\mathbb{R}}^{p^{k-1}}(\omega)$ and we call the resulting equation, Equation $k$. The coefficient of $\psi_{\mathbb{R}}^{p^{s+j}}(\omega)$ is $\left(\alpha_{j}^{\prime}-p^{t_{j}^{s}}\left(\delta_{j}-\theta \gamma_{s+j-k}^{k}\right)\right)$. For $j=k-s$, it is equal to $\left[\alpha_{k-s}^{\prime}-p^{t_{k-s}^{s}}\left(\delta_{k-s}-\theta \gamma_{0}^{k}\right)\right]$. It follows from Proposition 6.2.3 and Equation $k$ that $p^{t_{0}^{k}} \mid\left[\alpha_{k-s}^{\prime}-p^{t_{k-s}^{s}}\left(\delta_{k-s}-\theta \gamma_{0}^{k}\right)\right]$. $t_{k-s}^{s} \leqslant t_{0}^{k}$ by Lemma 4.1.2 and hence $p^{t_{k-s}^{s}} \mid p^{t_{0}^{k}}$, i.e. $p^{t_{k-s}^{s}} \mid\left[\alpha \prime_{k-s}-p^{t_{k-s}^{s}}\left(\delta_{k-s}-\theta \gamma_{0}^{k}\right)\right]$. Hence $p^{t_{k-s}^{s}} \mid \alpha \prime_{k-s}$, i.e. $\alpha_{k-s}^{\prime}=p^{t_{k-s}^{s}} \alpha_{k-s}$ for some $\alpha_{k-s} \in \mathbb{Z}$. The proposition now follows from (i) of the special case of the statement for $k=r_{n}$.

\section{Relations in the Group $J\left(L^{n}\left(p^{k}\right)\right)$}

\subsection{BACKGROUND MATERIAL ON $J\left(L^{n}\left(p^{k}\right)\right)$}

We, briefly, recall the results of [14]. Let $n, k \in \mathbb{Z}^{+}$and $p$ be a prime and $L^{n}\left(p^{k}\right)=$ $S^{2 n+1} / \mathbb{Z}_{p^{k}}$, where $\mathbb{Z}_{p^{k}}$ is the cyclic group of order $p^{k} . L^{n}\left(p^{k}\right)$ has the structure of a $C W$-complex, $L^{n}\left(p^{k}\right)=\cup_{j=0}^{2 n+1} e^{j}$ and its $(2 n)$ th-skeleton, $L_{0}^{n}\left(p^{k}\right)=\left\{\left[z_{0}, \ldots, z_{n}\right]\right.$ $\in L^{n}\left(p^{k}\right): z_{n}$ is real $\left.\geqslant 0\right\}$. We have an inclusion, $L^{n}\left(p^{k}\right) \subset P_{n}(\mathbb{C})$ and we denote by $\omega \in \tilde{K}_{\mathbb{R}}\left(L^{n}\left(p^{k}\right)\right)$ the pull-back of $\omega \in \tilde{K}_{\mathbb{R}}\left(P_{n}(\mathbb{C})\right)$. Then $\psi_{\mathbb{R}}^{p^{j}}(\omega)=0$ for $j \geqslant k$. Let $G(p, n, k)$ be the subgroup of $J\left(L^{n}\left(p^{k}\right)\right)$ generated by the powers of $\omega$, or, equivalently, by $\left\{\psi_{\mathbb{R}}^{p^{j}}(\omega)\right\}_{0 \leqslant j \leqslant k-1}$. For $p=2$, there is a line bundle $\xi$ on $L^{n}\left(2^{k}\right)$ such that $c(\xi)=\psi_{\mathbb{C}}^{2^{k-1}}(\eta)$, where $\eta$ is the complex Hopf-bundle over $L^{n}\left(2^{k}\right)$ and $c$ denotes complexification. Let $\lambda=\xi-1 \in \tilde{K}_{\mathbb{R}}\left(L^{n}\left(2^{k}\right)\right)$ denote its reduction. Then if $p$ is odd,

$$
J\left(L^{n}\left(p^{k}\right)\right)= \begin{cases}G(p, n, k), & \text { if } n \neq \equiv(\bmod 4), \\ G(p, n, k) \oplus \mathbb{Z}_{2}, & \text { if } n \equiv 0(\bmod 4) .\end{cases}
$$

If $p=2, J\left(L^{n}\left(2^{k}\right)\right)$ is generated additively by $\lambda$ and $G(2, n, k)$ subject to the relation: $2 \lambda=\psi_{\mathbb{R}}^{2^{k-1}}(\omega)$. Thus, the relations on $J\left(L^{n}\left(p^{k}\right)\right)$ will be completely determined if those on $G(p, n, k)$ are given. We shall call the relations on $G(p, n, k)$ fundamental relations. It follows from [14, Theorem 3.4.2] that $G(p, n, k)$ is isomorphic to $J_{p}\left(P_{n}(\mathbb{C})\right)$ for $k \geqslant r_{n}+1$. From now onwards we shall thus assume that $1 \leqslant k \leqslant r_{n}$.

\subsection{PRELIMINARIES ON $t_{0}^{s}(0 \leqslant s \leqslant k-1)$}

In this section, we shall adopt the results of Section 6.1 for lens spaces. 
DEFINITION 7.2.1. Let $p$ be a prime and $k, v \in \mathbb{Z}^{+}$. Define

$$
e(p, v, k)= \begin{cases}p^{\min \left(k, v_{p}(m(2 v))\right)} & \text { if } p \text { is odd }, \\ 2^{\min \left(k+1, v_{2}(m(2 v))\right)} & \text { if } p=2 .\end{cases}
$$

Note that this is [14, Definition 3.3.2]. We first modify Lemma 6.1.2.

LEMMA 7.2.2. Let $p$ be odd and $1 \leqslant k \leqslant r_{n}$. Then

$$
\sum_{s=0}^{k-1}\left(\left[\frac{n}{p^{s}(p-1)}\right]-\left[\frac{(n-1)}{p^{s}(p-1)}\right]\right)= \begin{cases}v_{p}\left(e\left(p, \frac{n}{2}, k\right)\right) & \text { if } n \text { is even }, \\ 0 & \text { if } n \text { is odd } .\end{cases}
$$

Proof. (i) If $n \not \equiv 0\left(\bmod (p-1)\right.$ then $\left[n / p^{s}(p-1)\right]=\left[(n-1) / p^{s}(p-1)\right]$. (ii) If $n \equiv 0(\bmod (p-1))$. Let $n=p^{\alpha}(p-1) \Delta,(\Delta, p)=1$. As in the proof of Lemma 6.1.2, for $0 \leqslant s \leqslant \alpha,\left[n / p^{s}(p-1)\right]-\left[(n-1) / p^{s}(p-1)\right]=1$ and $\left[n / p^{s}(p-1)\right]-\left[(n-1) / p^{s}(p-1)\right]=0$ for $s>\alpha$. Hence

$$
\begin{aligned}
& \sum_{s=0}^{k-1}\left(\left[\frac{n}{p^{s}(p-1)}\right]-\left[\frac{(n-1)}{p^{s}(p-1)}\right]\right)=\min (k, \alpha+1) \\
& \quad=\min \left(k, v_{p}(m(n))\right)=v_{p}\left(e\left(p, \frac{n}{2}, k\right)\right) .
\end{aligned}
$$

COROLLARY 7.2.3. Let $p$ be odd and $0 \leqslant k \leqslant r_{n}$. Then

$$
\sum_{s=0}^{k-1}\left[\frac{n}{p^{s}(p-1)}\right]=\sum_{v=1}^{[n / 2]} v_{p}(e(p, v, k)) .
$$

LEMMA 7.2.4. For $n$ even and $0 \leqslant k \leqslant r_{n}, \sum_{s=0}^{k-1}\left(\left[n / 2^{s}\right]-\left[(n-2) / 2^{s}\right]\right)=$ $v_{2}(e(2, n / 2, k))$.

COROLLARY 7.2.5. For $n$ even and $0 \leqslant k \leqslant r_{n}$,

$$
\sum_{s=0}^{k-1}\left[n / 2^{s}\right]=\sum_{v=1}^{n / 2} v_{2}(e(2, v, k)) .
$$

COROLLARY 7.2.6. For $n \equiv 1(\bmod 4)$ and $0 \leqslant k \leqslant r_{n}$,

$$
\sum_{s=0}^{k-1}\left[n / 2^{s}\right]=1+\sum_{v=1}^{(1 / 2)(n-1)} v_{2}(e(2, v, k)) .
$$

PROPOSITION 7.2.7. Except for $p=2$ and $n \equiv 3(\bmod 4)$ and for $0 \leqslant k \leqslant r_{n}$,

$$
|G(p, n, k)|=p^{\sum_{s=0}^{k-1} t_{0}^{s}}=p^{\sum_{s=0}^{k-1}\left[n / p^{s}(p-1)\right]} .
$$


Proof. For $n$ even, by [14, Corollary 3.3.9], $|G(p, n, k)|=\prod_{v=1}^{n / 2} e(p, v, k)$ and the result follows from this and Corollaries 7.2.3 and 7.2.5. The result for $n \equiv 1(\bmod 4)$ follows from

$$
|G(p, n, k)|= \begin{cases}|G(p, n-1, k)|, & p \text { odd }, \\ 2|G(2, n-1, k)|, & p=2\end{cases}
$$

as in the proof of [14, Theorem 3.4.2] and that

$$
\sum_{s=0}^{k-1}\left[\frac{n}{p^{s}(p-1)}\right]= \begin{cases}\sum_{s=0}^{k-1}\left[(n-1) / p^{s}(p-1)\right], & p \text { odd } \\ 1+\sum_{s=0}^{k-1}\left[(n-1) / 2^{s}\right], & p=2,\end{cases}
$$

and the case for $n$ even. For $n \equiv 3(\bmod 4)$ and $p$ odd, it follows from $|G(p, n, k)|=$ $|G(p, n-1, k)|$ as in the proof of [14, Theorem 3.4.2] and the fact that $\sum_{s=0}^{k-1}$ $\left[n / p^{s}(p-1)\right]=\sum_{s=0}^{k-1}\left[(n-1) / p^{s}(p-1)\right]$, and the case for $n$ even.

\subsection{RELATIONS IN $J\left(L^{n}\left(p^{k}\right)\right)$}

PROPOSITION 7.3.1. Let $1 \leqslant k \leqslant r_{n}$. There exist $k$-relations, $\sum_{h \geqslant 0}(-1)^{\varepsilon(h)}$ $p^{t_{j_{h}}^{s}} \psi_{\mathbb{R}}^{p^{s+j_{h}}}(\omega)=0(0 \leqslant s \leqslant k-1)$ in $G(p, n, k)$, where $\left\{j_{h}\right\}$ is the sequence constructed in Proposition 5.2.1

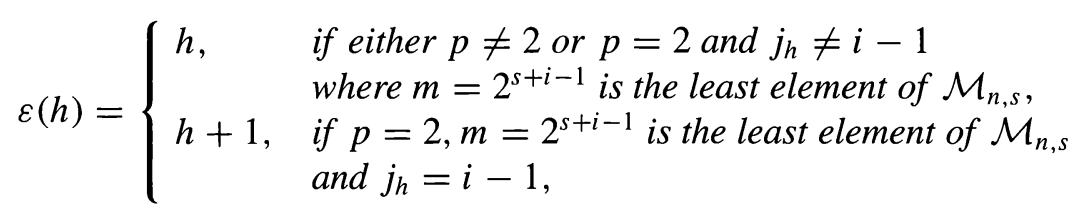

and this relation is complete except for $p=2$ and $n \equiv 3$.

Proof. These relations are the pull-back of the relations of Proposition 6.2.8 on $J_{p}\left(P_{n}(\mathbb{C})\right)$ via the inclusion, $L^{n}\left(p^{k}\right) \rightarrow P_{n}(\mathbb{C})$ where we note that $\psi_{\mathbb{R}}^{p^{i}}(\omega)=0$ on $J\left(L^{n}\left(p^{k}\right)\right)$ for $i \geqslant k$ and completeness follows from Proposition 7.2.7.

We shall now state the analogues of Proposition 6.2.10 and alternative proof of Proposition 3.3.1 for lens spaces.

DEFINITION 7.3.2. Let $0 \leqslant s \leqslant r_{n}, \iota: L^{n}\left(p^{s}\right) \rightarrow P_{n}(\mathbb{C})$ and for $k<s$ define

$$
\mathcal{J}(p, n, s ; k)=\iota^{!}(\mathcal{J}(p, n, k)) \subset G(p, n, s) \subset J\left(L^{n}\left(p^{s}\right)\right) .
$$

PROPOSITION 7.3.3. $\mathcal{J}(p, n, s ; k)=G\left(p,\left[n / p^{k}\right], s-k\right)$.

Proof. This proposition follows in exactly the same way from the $\alpha$-relation for lens spaces as Proposition 6.2.10 follows from the $\alpha$-relation for the complex projective space.

We now define a variation for lens spaces of the Atiyah-Todd number of [5]. 
DEFINITION 7.3.4. Let $M_{n}\left(p^{k}\right)=p^{v_{p}\left(M_{n}\left(p^{k}\right)\right)}$ where

$$
v_{p}\left(M_{n}\left(p^{k}\right)\right)=\sup _{1 \leqslant r \leqslant\left[\frac{n-1}{p-1}\right]}\left[r+\min \left(k-1, v_{p}(r)\right)\right] .
$$

PROPOSITION 7.3.5. The order of $\omega$ in $J\left(L^{n}\left(p^{k}\right)\right)$ is equal to $M_{n+1}\left(p^{k}\right)$.

Proof. This is [17, Theorem 1.1] for $p$ odd and [16, Theorem 1.2] for $p=2$ although the definition of the number $M_{n}\left(p^{k}\right)$ is worded slightly differently in these sources. Given our $J$-relations, its proof is a simple exercise in elementary algebra and would not take more than a few lines.

As a corollary to Definition 7.3.4 and Proposition 7.3.6, we obtain the following proposition, which is the counterpart for lens spaces of Proposition 6.2.11.

PROPOSITION 7.3.6. Let $n=p^{k}(p-1)+r(0 \leqslant r \leqslant p-2)$ and $1 \leqslant s \leqslant k$. Then $G(p, n, s)$ is generated by $\left\{\omega, \psi_{\mathbb{R}}^{p}(\omega), \ldots, \psi_{\mathbb{R}}^{p^{s-1}}(\omega)\right\}$ subject to the relations:

$$
\begin{aligned}
& p^{p^{k}}(\omega)=p^{\left(p^{k-1}-1\right)} \psi_{\mathbb{R}}^{p}(\omega), p^{p^{k-1}} \psi_{\mathbb{R}}^{p}(\omega)=p^{\left(p^{k-2}-1\right)} \psi_{\mathbb{R}}^{p^{2}}(\omega), \ldots, \\
& p^{p^{k-s+2}} \psi_{\mathbb{R}}^{p^{s-2}}(\omega)=(-1)^{(p-1) \delta_{s, k}} p^{\left(p^{k-s+1}-1\right)} \psi_{\mathbb{R}}^{p^{s-1}}(\omega), p^{p^{k-s+1}} \psi_{\mathbb{R}}^{p^{s-1}}(\omega)=0,
\end{aligned}
$$

where $\delta_{s, k}$ is the Kronecker delta.

From Proposition 7.3.8 we obtain the primary-decomposition of $G(p, n, s)$ for $n=p^{k}(p-1)+r(0 \leqslant r \leqslant p-2)$.

PROPOSITION 7.3.7. Let $n=p^{k}(p-1)+r(0 \leqslant r \leqslant p-2)$ and $1 \leqslant s \leqslant k$. Then $G(p, n, s)=\mathbb{Z}_{p^{p^{k}+s-1}} \oplus \mathbb{Z}_{p^{\left(p^{k-1}-1\right)}} \oplus \cdots \oplus \mathbb{Z}_{p^{\left(p^{k-s+1}-1\right)}}$.

Proof. As in the proof of Proposition 6.2.12, let $x_{0}=\omega$,

$$
\begin{aligned}
x_{1} & =\psi_{\mathbb{R}}^{p}(\omega)-p^{\left(p^{k}-p^{k-1}+1\right)} \omega \cdots x_{s-1} \\
& =\psi_{\mathbb{R}}^{p^{s-1}}(\omega)-(-1)^{(p-1) \delta_{s, k}} p^{\left(p^{k-s+2}-p^{k-s+1}+1\right)} \psi_{\mathbb{R}}^{p^{s-2}}(\omega) .
\end{aligned}
$$

Then $\left\{x_{0}, x_{1}, \ldots, x_{s-1}\right\}$ forms a basis for $G(p, n, s)$. The order of $x_{0}$ is $M_{n+1}\left(p^{s}\right)$ by Proposition 7.3.6, where $v_{p}\left(M_{n+1}\left(p^{s}\right)\right)=p^{k}+s-1$ and the orders of $x_{1}, \ldots$, $x_{s-1}$ are as in the proof of Proposition 6.2.12.

PROPOSITION 7.3.8. Let $n=p^{k}(p-1)+r(0 \leqslant r \leqslant p-2,1 \leqslant s \leqslant k)$. Then

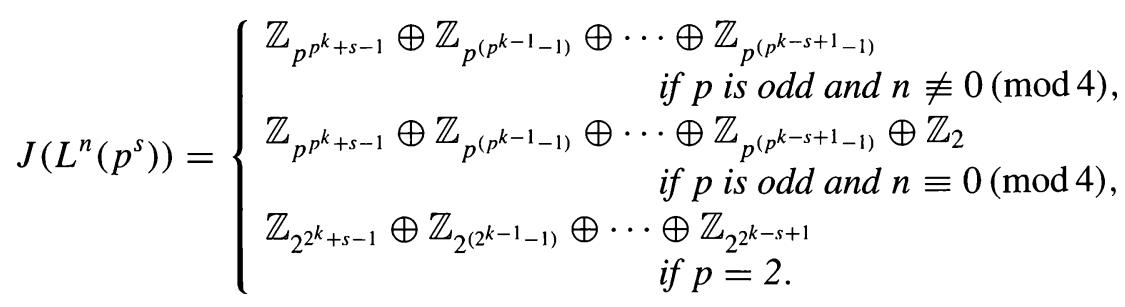


Proof. The case of $p$ odd follows from the discussion in Section 7.1 and Proposition 7.3.7. In the case $p=2$ let $\lambda$ be the reduction of the canonical line bundle over $L^{n}\left(2^{s}\right)$. Since we have the relation, $2 \lambda=\psi_{\mathbb{R}}^{2^{s-1}}(\omega)$, the generator $x_{s-1}$ of the last summand in $G(2, n, s)$ is replaced by $x_{s-1}^{\prime}=(1 / 2) x_{s-1}=$ $\lambda-(-1)^{\delta_{s, k}} 2^{\left(2^{k-s+2}-2^{k-s+1}\right)} \psi_{\mathbb{R}}^{2^{s-2}}(\omega)=\lambda-(-1)^{\delta_{s, k}} 2^{2^{k-s+1}} \psi_{\mathbb{R}}^{2^{s-2}}(\omega)$ whose order is twice that of $x_{s-1}$; i.e. equal to $2^{2^{k-s+1}}$.

\section{The Algebraic Viewpoint and the Groups $D(\alpha, \beta ; n)$}

In this section, we shall show, briefly, without going into proofs that the group $J\left(P_{n}(\mathbb{C})\right)$ is a subgroup of a larger group $D_{n}$ which arises naturally in algebra and which is first introduced in [13].

\subsection{WEAK $D$-SERIES}

DEFINITION 8.1.1. For each prime $p$, let $\alpha_{p}, \beta_{p} \in \mathbb{Z}^{+}$and $\alpha=\left(\alpha_{p}\right)$ and $\beta=$ $\left(\beta_{p}\right)$. We call a rational series, $f(x)= \pm 1+\sum_{j \geqslant 1} a_{j} x^{j}\left(a_{j} \in \mathbb{Q}\right)$ a weak $D$-series of type $(\alpha, \beta)$ if and only if $v_{p}\left(a_{k}\right) \geqslant-\beta_{p}\left[k / \alpha_{p}\right] \forall k \in \mathbb{Z}^{+}$. Note that this is [13, Definition 3.1].

\subsection{THE GROUP $D(\alpha, \beta ; n)$}

Let $\tilde{D}(\alpha, \beta)$ be the set of weak $D$-series of type $(\alpha, \beta)$. Then it follows from [13, Observation 5.1] that $\tilde{D}(\alpha, \beta)$ is a multiplicative group. Let $\mathbb{Z}^{*}[[x]]$ be the group of series with integer coefficients and with constant term \pm 1 . Let $D(\alpha, \beta ; n)$ and $\mathbb{Z}_{n, X}$ be the images of $\tilde{D}(\alpha, \beta)$ and $\mathbb{Z}^{*}[[x]]$ under the quotient map, $\mathbb{Q}[[x]] \rightarrow$ $\mathbb{Q}[[x]] /\left(x^{n+1}\right)$. Since $\mathbb{Z}^{*}[[x]] \subset \tilde{D}(\alpha, \beta)$ we have $\mathbb{Z}_{n, X} \subset \tilde{D}(\alpha, \beta)$. Define $D(\alpha, \beta ; n)=\tilde{D}(\alpha, \beta) / \mathbb{Z}_{n, X}$. The highest invariants of $D(\alpha, \beta ; n)$ are computed in [13, Proposition 5.2].

DEFINITION 8.2.1. We define the Adams operation $\psi^{k}$ on $\mathbb{Q}[[x]]$ by

$$
\psi^{k}(x)=(1+x)^{k}-1=k x+\left(\begin{array}{l}
k \\
2
\end{array}\right) x^{2}+\left(\begin{array}{l}
k \\
3
\end{array}\right) x^{3}+\cdots+k x^{k-1}+x^{k} .
$$

Then $\psi^{k}$ is a ring homomorphism on $\mathbb{Q}[[x]]$ which preserves $\tilde{D}(\alpha, \beta), \mathbb{Z}^{*}[[x]]$ and $\left(x^{n+1}\right)$. Thus $\psi^{k}$ passes to the quotient and acts on $\mathbb{Q}[[x]] /\left(x^{n+1}\right)$ and this action preserves $\tilde{D}(\alpha, \beta ; n)$ and $\mathbb{Z}_{n, X}$. Hence $\psi^{k}$ further passes to the quotient and acts on $D(\alpha, \beta ; n)$ as a group homomorphism.

DEFINITION 8.2.2. Let $D_{n}=D(\alpha, \beta ; n)$ for $\alpha_{p}=p-1$ and $\beta_{p}=1$. This group should play an important role in elementary number theory. 


\subsection{THE ADAMS CONJECTURE FOR $D_{n}$}

For $k \in \mathbb{Z}^{+}$, let $\hat{Q}_{k} \subset \mathbb{Q}$ be the sub-ring of rationals whose denominators are prime to $k$ and $\hat{Q}_{k}^{*}[[x]]$ be the group series with coefficients in $\hat{Q}_{k}$ and with constant term $\mp$. Then $\mathbb{Z}^{*}[[x]] /\left(x^{n+1}\right) \subset \hat{Q}_{k}^{*}[[x]] /\left(x^{n+1}\right)$. Denote their quotient by $\mathbb{Z}_{k, n}$.

DEFINITION 8.3.1. $f \in D_{n}$ satisfies the Adams conjecture if and only if $\psi^{k}(f) /$ $f \in \mathbb{Z}_{k, n}, \forall k \in \mathbb{Z}^{+}$. Those elements of $D_{n}$ which satisfy the Adams conjecture form a subgroup $A_{n}$ and $\psi^{k}$ preserves $A_{n}$. Let $J_{\mathbb{C}}\left(P_{n}(\mathbb{C})\right)$ be the subgroup defined in [4] so that $J_{\mathbb{C}}\left(P_{n}(\mathbb{C})\right)=J\left(P_{n}(\mathbb{C})\right)$ for $n \not \equiv 1(\bmod 4)$ and $J_{\mathbb{C}}\left(P_{n}(\mathbb{C})\right)=$ $J\left(P_{n-1}(\mathbb{C})\right)$ for $n \equiv 1(\bmod 4)$.

THEOREM 8.3.2. There exists an isomorphism, $\sigma: J_{\mathbb{C}}\left(P_{n}(\mathbb{C})\right) \rightarrow A_{n}$ which is a $\psi^{k}$-map given by $\sigma(\mu)=\log (1+x) / x$ (and hence $\left.\sigma\left(\psi_{\mathbb{C}}^{k}(\mu)\right)\right)=\log (1+$ $\left.\psi^{k}(x)\right) / x$, where $\mu=\eta-1 \in \tilde{K}_{\mathbb{C}}\left(P_{n}(\mathbb{C})\right)$.

Thus, $A_{n}=J_{\mathbb{C}}\left(P_{n}(\mathbb{C})\right)$. One may also localise $\sigma$ at different primes $p$. Let $J_{\mathbb{C}}\left(P_{n}(\mathbb{C})\right)_{p}$ and $A_{n}(p)$ be the $p$-components of these groups. One can define an isomorphism, $\sigma_{p}: J_{\mathbb{C}}\left(P_{n}(\mathbb{C})\right)_{p} \rightarrow A_{n}(p)$ which is a $\psi^{k}$-map by

$$
\sigma_{p}(\mu)=\frac{\psi^{p}(x)}{p x}=1+\frac{(p-1)}{2 !} x+\frac{(p-1)(p-2)}{3 !} x^{2}+\cdots+\frac{1}{p} x^{p-1}
$$

(and, hence, $\left.\sigma_{p}\left(\psi_{\mathbb{C}}^{k}(\mu)\right)=\psi^{p}\left(\psi^{k}(x)\right) / p x=\psi^{k p}(x) / p x\right)$. One may, equivalently, use the truncated power series, $L_{p}(x)=x+x^{p} / p+x^{p^{2}} / p^{2}+\cdots+x^{p^{n}} / p^{n}$, instead of $\psi^{p}(x) / p x$, i.e. define an isomorphism, $\sigma_{p}^{\prime}: J_{\mathbb{C}}\left(P_{n}(\mathbb{C})\right)_{p} \rightarrow A_{n}(p)$ by $\sigma_{p}^{\prime}(\mu)=L_{p}(x) / x\left(\right.$ and $\sigma_{p}^{\prime}\left(\psi_{\mathbb{C}}^{k}(\mu)\right)=L_{p}\left(\psi^{k}(x)\right) / x$. $)$

\subsubsection{Generalisations of the J-groups of complex projective spaces}

The Adams operation $\psi^{k}$ acts on the finite Abelian group $D(\alpha, \beta ; n)$ for any $\alpha=\left(\alpha_{p}\right)$ and $\beta=\left(\beta_{p}\right)$, not just $D_{n}$. We can let $A(\alpha, \beta ; n)$ be the subgroup of $D(\alpha, \beta ; n)$ of elements which satisfy the Adams conjecture. Then $A(\alpha, \beta ; n)$ provides a natural generalisation of the $J$-groups of complex projective spaces. It is of interest to see if $A(\alpha, \beta ; n)$ carries any geometric significance for arbitrary $\alpha$ and $\beta$.

\subsubsection{Problems}

The complete determination of the group $D_{n}$ (or, more generally, $D(\alpha, \beta ; n)$ ) is a formidable problem. Our methods may perhaps be adapted to its solution. The quotient group, $D_{n} / J_{\mathbb{C}}\left(P_{n}(\mathbb{C})\right)$ or, more generally, $D(\alpha, \beta ; n) / A(\alpha, \beta ; n)$, will give a measure of how far truncated weak $D$-series deviate from satisfying the Adams conjecture. 


\section{References}

1. Adams, J. F.: On the groups $J(X)$-II, J. Topology 3 (1965), 137-172.

2. Adams, J. F.: On the groups $J(X)-$ III, J. Topology 3 (1965), 193-222.

3. Adams, J. F.: Infinite Loop Spaces, Princeton Univ. Press, Princeton, NJ, 1978.

4. Adams, J. F. and Walker G.: On complex Stiefel manifolds, Proc. Cambridge Philos. Soc. 61 (1965), 81-103.

5. Atiyah, M. F. and Todd, J. A.: On complex Stiefel manifolds, Proc. Cambridge Philos. Soc. 56 (1960), 342-353.

6. Becker, J. C. and Gottlieb, D. H.: Transfer map for fibre bundles, J. Topology 14 (1975), 1-12.

7. Bott, R.: Lectures on $K(X)$, W. A. Benjamin, New York, 1969.

8. Crabb, M. C. and Knapp, K.: On the codegree of negative multiples of the Hopf bundle, Proc. Royal Soc. Edinburgh A 107 (1987), 87-107.

9. Crabb, M. C. and Knapp, K.: James numbers, Math. Ann. 282 (1988), 395-422.

10. Dibag, I.: Degree functions $q$ and $q^{\prime}$ on the group $J_{S O}(X)$, Habilitationsschrift, Middle East Technical University, Ankara, 1977.

11. Dibag, I.: Degree-theory for spherical fibrations, Tohoku Math. J. 34 (1982), 161-177.

12. Dibag, I.: On the Adams conjecture, Proc. Amer. Math. Soc. 87 (1983), 367-374.

13. Dibag, I.: Integrality of rational D-series, J. Algebra 164(2) (1994), 468-480.

14. Dibag, I.: J-Approximation of complex projective spaces by lens spaces, Pacific J. Math. 191(2) (1999), 223-241.

15. Fujii, K., Kobayashi, T., Shimumura, K. and Sugawara, M.: KO-Groups of lens spaces modulo powers of two, Hiroshima Math. J. 8 (1978), 469-489.

16. Fujii, K.: $J$-groups of lens spaces modulo powers of two, Hiroshima Math J. 10 (1980), 659-690.

17. Fujii, K. and Sugawara, M.: The order of the canonical element in the $J$-group of the lens space, Hiroshima Math J. 10 (1980), 369-374.

18. Kambe, T.: The structure of $K_{\wedge}$-rings of the lens spaces and their applications, J. Math. Soc. Japan 18(2) (1966), 135-146.

19. Kobayashi, T., Murakami, S. and Sugawara, M.: Note on $J$-groups of lens spaces, Hiroshima Math. J. 7 (1977), 387-409.

20. Lam, K. Y.: Fibre homotopic trivial bundles over complex projective spaces, Proc. Amer. Math. Soc. 33 (1972), 211-212. 